\title{
HYPERBOLIC TRIANGLES WITHOUT EMBEDDED EIGENVALUES
}

\author{
LUC HILLAIRET AND CHRIS JUDGE
}

\begin{abstract}
We consider the Neumann Laplacian acting on square-integrable functions on a triangle in the hyperbolic plane that has one cusp. We show that the generic such triangle has no eigenvalues embedded in its continuous spectrum. To prove this result we study the behavior of the real-analytic eigenvalue branches of a degenerating family of triangles. In particular, we use a careful analysis of spectral projections near the crossings of these eigenvalue branches with the eigenvalue branches of a model operator.
\end{abstract}

\section{INTRODUCTION}

Though well-studied for over fifty years, the spectral theory of hyperbolic surfaces still presents basic unresolved questions Sarnak03. For example, does there exist a noncompact, finite area hyperbolic surface whose Laplacian has no nonconstant square-integrable eigenfunctions? This question has been the subject of many investigations including [ColinDeVerdière83, PhlSrn85, [DIPS85, PhlSrn92a, Wolpert92, Wolpert94, and [PhlSrn94.

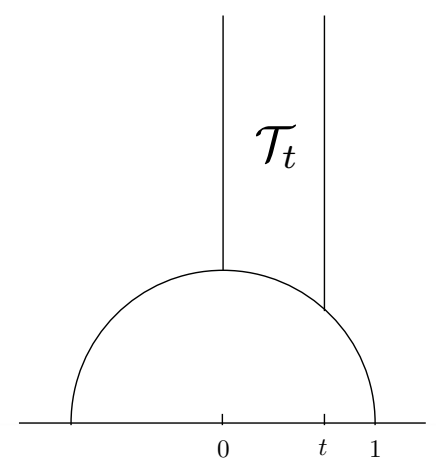

Figure 1 . The triangle $\mathcal{T}_{t}$ defined by $x^{2}+y^{2} \geqslant 1$ and $0 \leqslant x \leqslant t$.

As a model problem, Phillips and Sarnak PhlSrn92b] suggested studying the Neumann eigenvalue problem on the domain $\mathcal{T}_{t} \subset \mathbb{R} \times \mathbb{R}^{+}$pictured in Figure 1 In this paper, we prove the following:

The work of L.H. has been partly supported by the ANR programs METHCHAOS, NOSEVOL and Gerasic-ANR-13-BS01-0007-0.

The work of C.J. has been partly supported by a Simons collaboration grant. C.J. Also thanks l'Université d'Orleans for its hospitality. 
Theorem 1.1. For all but at most countably many $t \in] 0,1[$, the Neumann Laplacian on the geodesic triangle $\mathcal{T}_{t}$ in the hyperbolic plane has no nonconstant (squareintegrable) eigenfunction.

The group $G_{t}$ of hyperbolic isometries generated by reflections in the geodesic arcs that bound $\mathcal{T}_{t}$ is discrete if and only if $t=\cos (\pi / n)$ for $n \geqslant 3$ an integer. For example, if $t=1 / 2$, then $G_{t}$ contains an index two subgroup that is naturally isomorphic to $P S L_{2}(\mathbb{Z})$. It follows from the seminal work of A. Selberg Selberg that if $n=3,4$, or 6 , then the Neumann Laplacian has infinitely many nonconstant eigenfunctions. In particular, for some special $t$, there do exist square-integrable solutions to the Neumann problem 1

In [Jdg95, Theorem 1.1] was verified under an additional - and as of yet unjustifiedassumption concerning the spectral multiplicities of the Neumann Laplacian acting on $L^{2}\left(\mathcal{T}_{1}\right)$. The proof consisted of studying the singular perturbation problem associated with letting $t$ tend to 1 . Similar singular perturbations were studied in the context of degenerating hyperbolic surfaces Wolpert94 and unitary characters over a fixed hyperbolic surface [PhlSrn94]. In Wolpert94], [PhlSrn94], [Jdg95], and all prior work on this problem, it was necessary to make assumptions about the multiplicities of the spectrum of the limiting surface.

The angles of a geodesic triangle in the hyperbolic plane determine the isometry class of the triangle. The angles of $\mathcal{T}_{t}$ are $(\pi / 2, \arccos (t), 0)$. It is not difficult to extend Theorem 1.1 to triangles with angles $\left(\theta_{1}, \theta_{2}, 0\right)$ (see $\$ 4$ ).

Theorem 1.2. The set of $\left(\theta_{1}, \theta_{2}\right)$ for which the hyperbolic triangle with angles $\left(\theta_{1}, \theta_{2}, 0\right)$ admits a nonconstant Neumann Laplace eigenfunction has Lebesgue measure zero and is contained in a countable union of nowhere dense sets.

In other words, the generic hyperbolic triangle with one cusp has no non-constant Neumann eigenvalue where 'generic' can be taken in both a topological and a measurable sense. Theorem 1.1 gives the existence of a triple of angles $\left(\theta_{1}, \theta_{2}, 0\right)$ for which there are no nonconstant Neumann eigenfunctions. Theorem 1.2 then results from applying a general and well-understood principle concerning analytic perturbations (See, for example, HlrJdg09]). On the other hand, the proof of Theorem 1.1 is much more involved. In particular, the proof will rely upon a refined analysis of 'crossings' of eigenvalue branches.

To prove Theorem 1.1, we further develop the method of asymptotic separation of variables that we introduced in [HlrJdg11] to study generic simplicity of eigenvalues. This method facilitates the study of real-analytic eigenvalue branches in situations where a geometric domain degenerates onto a lower dimensional domain. There is a vast literature - for instance, BorFre10, GriJer96], FriSol09]concerning perturbations involving degeneration onto lower dimensional domains, but most of these studies do not address analytic eigenvalue branches. In contrast, our results depend crucially on a study of real-analytic eigenbranches and their crossings.

1.1. An outline of this paper. We now describe the content of each section.

\footnotetext{
${ }^{1}$ In the case where $n \geqslant 3$ is an integer not equal to 3,4 , or 6 , Phillips and Sarnak asked whether the domain $\mathcal{T}_{\cos (\pi / n)}$ has no nonconstant Neumann eigenfunctions [PhlSrn92b]. We should point out that since Theorem 1.1 allows for countably many exceptional $t$, it does not directly answer their question.
} 
In $₫ 2$ we establish notation and recall basic features of the spectral theory of the Laplacian acting on functions on a domain in the hyperbolic plane having one cusp. We describe the Fourier decomposition associated to the cusp. The zeroth Fourier mode is responsible for an essential spectrum of $\left[\frac{1}{4}, \infty[\right.$. Following [LaxPhl] and ColinDeVerdière82, we will replace the Dirichlet quadratic form $\mathcal{E}(u)=\langle\Delta u, u\rangle$ with a modification $\mathcal{E}_{\beta}$ obtained by 'truncating' the zeroth Fourier coefficient above $y=\beta$. An eigenfunction $u$ of $\mathcal{E}_{\beta}$ corresponds to an eigenfunction of $\mathcal{E}$ if and only if the zeroth Fourier coefficient of $u$ vanishes identically. We will call such an eigenfunction a cusp form $\left.\right|^{2}$ The operator associated to $\mathcal{E}_{\beta}$ has compact resolvent and hence discrete spectrum. This makes $\mathcal{E}_{\beta}$ a much better candidate for the application of methods from spectral perturbation theory.

In $\$ 3$ we recall and make precise some ideas familiar in the perturbational study of cusp form existence. We consider a real-analytic family, $t \mapsto q_{t}$, of quadratic forms that have the same domain as $\mathcal{E}_{\beta}$. We say that a real-analytic family $t \mapsto u_{t}$ of eigenfunctions of $q_{t}$ is a cusp form eigenbranch if and only $u_{t}$ is a cusp form for each $t$. We demonstrate a dichotomy: Either the family $t \mapsto q_{t}$ has a real-analytic cusp form eigenbranch or the set of $t$ such that $q_{t}$ has a cusp form is countable.

In 84 , we consider arbitrary real-analytic paths in the space of hyperbolic triangles with one cusp. We apply the results of $\$ 3$ to deduce Theorem 1.2 under the assumption that there exists a triangle with no nonconstant Neumann eigenfunction. The remainder of the paper is devoted to proving Theorem 1.1 which will give the existence of such a triangle.

In $\$ 5$ we specialize to the family $\mathcal{T}_{t}$. After renormalizing by a factor of $t^{2}$, we find that for each $u$, the function $t \mapsto q_{t}(u)$ has a Taylor expansion at $t=0$. We compute the leading order terms of this expansion.

In $\$ 6$ we show that the method of the asymptotic separation of variables introduced in HlrJdg11 may be used to analyse the family of quadratic forms $q_{\beta, t}$. In particular, we define a reference quadratic form $a_{t}$ to which separation of variables applies and that is asymptotic to $q_{\beta, t}$ at 'first order'. By separation of variables we mean that each eigenfunction of $a_{t}$ is of the form $v_{t}^{\ell}(y) \cdot \cos (\pi \ell x)$ with $\ell \in \mathbb{Z}$ and $v_{t}^{\ell}$ a solution to

$$
-t^{2} \cdot u^{\prime \prime}+\left((k \pi)^{2}-\frac{\lambda}{y^{2}}\right) \cdot u=0
$$

a renormalized form of the equation for a modified Bessel function with imaginary parameter. The potential $(k \pi)^{2}-\lambda \cdot y^{-2}$ is positive for $y$ large and has a unique zero at $y=\sqrt{\lambda} /(k \pi)$. To analyse the solutions to $(1)$, we will relate them to the Airy functions, solutions to the ordinary differential equation $\partial_{x}^{2} A=x \cdot A=0$. The remainder of the paper depends crucially on the analysis of (1) using Airy functions that has been placed in the Appendices.

In $\$ 7$ we prove a non-concentration estimate-Proposition 7.2 and use this estimate to derive information concerning the real-analytic eigenbranches $\left(E_{t}, u_{t}\right)$ of $q_{\beta, t}$. First, we show that there exists an integer $k$ so that $E_{t}$ limits to $(\pi k)^{2}$ as $t$ tends to zero. Second, we find that if the spectral projection of $u_{t}$ onto the space $V_{k}$ spanned by functions of the form the $\psi(y) \cdot \cos (\pi k x)$ is relatively small, then

\footnotetext{
${ }^{2}$ For $t=\cos (\pi / n)$, these are 'cusp forms' in the sense of the theory of automorphic forms, but otherwise there is no discrete group, and hence they are not cusp forms in the traditional sense. In this paper we will always be considering 'even' cusp forms, that is, eigenfunctions satisfying Neumann conditions.
} 
the derivative $\partial_{t} E_{t}$ is of order $1 / t$. Finally, we show that if $\left(E_{t}, u_{t}\right)$ is a cusp form eigenbranch, then $E_{t}$ can not limit to zero.

In 88 we prove Theorem 1.1. By the dichotomy of 8 , it suffices to show that real-analytic cusp form eigenbranches do not exist. We suppose to the contrary that the real-analytic family $q_{\beta, t}$ has a cusp form eigenbranch $\left(E_{t}, u_{t}\right)$. By the results of 97 . we have that $E_{t}$ limits to $(\pi k)^{2}$ where $k \in \mathbb{Z}^{+}$. By improving the analysis of HlrJdg11, we show that there exists an eigenbranch, $\lambda_{t}^{*}$, of $a_{t}$ that 'tracks' $E_{t}$ at order $t$ in the sense that

$$
\limsup _{t \rightarrow 0} \frac{1}{t} \cdot\left|E_{t}-\lambda_{t}^{*}\right|<\infty .
$$

We will obtain a contradiction to this estimate by estimating $f(t):=\frac{d}{d t}\left(E_{t}-\lambda_{t}^{*}\right)$ from below.

Indeed, we show that when the norm of the projection $w_{t}^{k}$ of $u_{t}$ onto $V_{k}$ is relatively large with respect to $\left\|u_{t}\right\|$, the function $f(t)$ is of order $t^{-\frac{1}{3}}$, whereas when $\left\|w_{t}^{k}\right\|$ is relatively small, the function $f(t)$ is of order $t^{-1}$. By controling the sizes of the sets where $\left\|w_{t}^{k}\right\|$ is respectively small and large relative to $\left\|u_{t}\right\|$ and by integrating, we will contradict (2).

The key observation is the following: Since $E_{t}$ limits to $(k \pi)^{2}$, it has to 'cross' each of the eigenbranches of $a_{t}$ that limit to zero. We show that near such a crossing, the branch $u_{t}$ must 'interact' with the functions in $V_{0}$ to such an extent that the projection onto $V_{k}$ cannot be too large. The effect of each interaction is made precise by careful estimates of the off-diagonal terms in the quadratic form $q_{t}-a_{t}$ (Appendix A). By summing the effects of these interactions, we eventually prove that there exists $c>0$ so that

$$
E_{t}-\lambda_{t}^{*} \geqslant c \cdot t^{\frac{2}{3}}
$$

thus contradicting (2).

\section{The Spectrum of a domain in the hyperbolic Plane With A Cusp}

In this section, we describe some basic spectral theory of the Neumann Laplace operator acting on the square-integrable functions on domains in the hyperbolic plane with a cusp. We define the Laplacian and associated Dirichlet quadratic form, describe the Fourier decomposition of eigenfunctions along horocycles, construct a modification of the Dirichlet form whose eigenfunctions include the eigenfunctions (cusp forms) of the standard Laplacian but whose spectrum is discrete.

2.1. The quadratic forms associated to the Neumann Laplacian. The halfplane $\{(x, y), y>0\}$ equipped with the Riemannian metric $y^{-2}\left(d x^{2}+d y^{2}\right)$ is the Poincaré-Lobachevsky model for the 2-dimensional hyperbolic space $\mathbb{H}^{2}$. The measure associated to the Riemannian metric $g=y^{-2}\left(d x^{2}+d y^{2}\right)$ is given by integrating

$$
d m=\frac{d x d y}{y^{2}} .
$$

In the present context, a cusp of width $w$ and height $y_{0}$ is the subset $S_{w, y_{0}}:=$ $[0, w] \times\left[y_{0}, \infty\left[\right.\right.$ of the upper half plane. A domain $\Omega \subset \mathbb{H}^{2}$ is said to have one cusp if $\Omega$ is the union of a cusp and a compact set. We will assume that the boundary of $\Omega$ is the union of finitely many geodesic arcs and that the interior of $\Omega$ is connected. 
Let $\mathcal{D}(\bar{\Omega})$ denote the set of functions $u: \Omega \rightarrow \mathbb{C}$ such that $u$ is the restriction to $\Omega$ of a compactly supported smooth function defined on some neighbourhood of $\Omega$.

The $L^{2}$-inner product of two functions $u$ and $v$ in $\mathcal{D}(\bar{\Omega})$ is defined by

$$
\mathcal{N}(u, v):=\int_{\Omega} u(x, y) \cdot \overline{v(x, y)} d m .
$$

Abusing notation slightly, we will often write $\mathcal{N}(u)$ in place of $\mathcal{N}(u, u)$. Let $L^{2}(\Omega, d m)$ denote the completion of $\mathcal{D}(\bar{\Omega})$ with respect to the norm $\mathcal{N}(u)^{\frac{1}{2}}$.

To define the Neumann Laplacian we consider the bilinear form defined on $\mathcal{D}(\bar{\Omega})$ by

$$
\mathcal{E}(u, v):=\int_{\Omega} g(\nabla u, \nabla v) d m
$$

where $\nabla$ satisfies $g(\nabla f, X)=X f$ for all vector fields $X$ and smooth functions $f$. Let $\mathcal{E}(u)$ denote the value of the quadratic form $u \mapsto \mathcal{E}(u, u)$. One computes that

$$
\mathcal{E}(u)=\int_{\Omega}\left|\partial_{x} u(x, y)\right|^{2}+\left|\partial_{y} u(x, y)\right|^{2} d x d y .
$$

Let $H^{1}(\Omega)$ denote the completion of $\mathcal{D}(\bar{\Omega})$ with respect to the norm $(\mathcal{E}(u)+\mathcal{N}(u))^{\frac{1}{2}}$. We will consider $\mathcal{E}$ as a nonnegative symmetric bilinear form on $L^{2}(\Omega)$ with domain $H^{1}(\Omega)$. As such it is densely defined and closed, and hence there exists a unique, densely defined, self-adjoint operator $\Delta$ on $L^{2}(\Omega)$ such that for each $v \in H^{1}(\Omega)$ and $u$ in the domain of $\Delta$ we have 3

$$
\mathcal{N}(\Delta u, v)=\mathcal{E}(u, v) .
$$

The operator $\Delta$ is called the Neumann Laplacian. It can be shown that $u \in H^{1}(\Omega)$ if and only if $u \in L^{2}(\Omega, d m)$ and $\mathcal{E}(u)<+\infty$ where in the definition of $\mathcal{E}$ the partial derivatives are to be taken in the distributional sense.

It is well-known that $\Delta$ has essential spectrum equal to $[1 / 4, \infty[$. (For example, see [LaxPhl] or ColinDeVerdière82]). Apart from this essential spectrum, $\Delta$ may also have eigenvalues either smaller than $1 / 4$ or embedded in the continuous spectrum.

From (4) we see that $u$ is an eigenfunction of $\Delta$ with eigenvalue $E$ if and only if $u \in H^{1}(\Omega)$ and for each $v \in H^{1}(\Omega)$

$$
\mathcal{E}(u, v)=E \cdot \mathcal{N}(u, v) .
$$

2.2. Fourier decomposition in the cusp. For each positive integer $k$, define

$$
e_{k}(x):=2^{\frac{1}{2}} \cdot \cos (k \pi \cdot x)
$$

and define $e_{0} \equiv 1$. The collection $\left\{e_{k} \mid k \geqslant 0\right\}$ is an orthonormal basis for $L^{2}([0,1])$. Hence, the functions

$$
x \mapsto \frac{1}{\sqrt{w}} \cdot e_{k}\left(\frac{x}{w}\right)
$$

provide an orthonormal basis of $L^{2}([0, w])$.

For positive $w, y_{0}$, let $S_{w, y_{0}}=[0, w] \times\left[y_{0}, \infty[\right.$ be a cusp of width $w$ and height $y_{0}$.

\footnotetext{
${ }^{3}$ See, for example, Theorem VI.2.1 Kato.
} 
For each $u$ in $L^{2}\left(S_{w, y_{0}}, d m\right)$ and almost every $y \geqslant y_{0}$, the function $x \mapsto u(x, y)$ belongs to $L^{2}([0, w])$. Thus we can write

$$
u(x, y)=\sum_{k \geqslant 0} u^{k}(y) \cdot e_{k}\left(\frac{x}{w}\right)
$$

where

$$
u^{k}(y):=\frac{1}{w} \int_{0}^{w} u(x, y) \cdot e_{k}\left(\frac{x}{w}\right) d x .
$$

belongs to $L^{2}\left(\left[y_{0}, \infty\left[, y^{-2} d y\right)\right.\right.$. We refer to $u^{k}$ as the $k^{\text {th }}$ Fourier coefficient of $u$. More generally, if $\Omega$ is a domain with a cusp $S_{w, y_{0}}$, then we define the $k^{\text {th }}$ Fourier coefficient of a function $v: \Omega \rightarrow \mathbb{C}$ to be the $k^{\text {th }}$ Fourier coefficient restriction of $v$ to $S_{w, y_{0}}$. Parseval's theorem gives

$$
\mathcal{N}\left(u \cdot \mathbb{1}_{\left[y_{0}, \infty[\right.}\right)=\sum_{k \geqslant 0} \int_{y_{0}}^{\infty}\left|u^{k}(y)\right|^{2} \frac{d y}{y^{2}}
$$

where $\mathbb{1}_{X}$ denotes the characteristic function of a set $X$.

Lemma 2.1. If $u \in H^{1}(\Omega, d m)$ is a Neumann eigenfunction of $\mathcal{E}$ with eigenvalue $E$, then for each $k \in \mathbb{N}$ and each $y>y_{0}$, the Fourier coefficient $u^{k}$ satisfies

$$
-\left(u^{k}\right)^{\prime \prime}+\left(\frac{(k \pi)^{2}}{w^{2}}-\frac{E}{y^{2}}\right) u^{k}=0 .
$$

Proof. If $v$ is a smooth function on $\Omega$, then since $u$ is a Neumann eigenfunction of $\mathcal{E}$, integration by parts gives

$$
-\int_{\Omega}\left(u \cdot \partial_{x}^{2} v+u \cdot \partial_{y}^{2} v\right) d x d y=E \int_{\Omega} u \cdot v \frac{d x d y}{y^{2}} .
$$

By letting $v=\phi(y) \cdot e_{k}(x / w)$ where $\phi$ is a smooth function with compact support in $] y_{0}, \infty[$, we find that

$$
\frac{(k \pi)^{2}}{w^{2}} \int_{y_{0}}^{\infty} u^{k}(y) \cdot \phi(y) d y-\int_{y_{0}}^{\infty} u^{k}(y) \cdot \phi^{\prime \prime}(y) d y=E \int_{y_{0}}^{\infty} u^{k}(y) \cdot \frac{d y}{y^{2}} .
$$

It follows that $u^{k}$ satisfies $(8)$ in the distributional sense in $\mathcal{D}^{\prime}\left(\left(y_{0}, \infty\right)\right)$. By elliptic regularity, $u^{k}$ is actually smooth and satisfies $(8)$ in the strong sense.

In particular, $u^{0}=A y^{s}+B y^{1-s}$ for some constants $A$ and $B$ with $E=s(1-s)$. If $E \geqslant 1 / 4$, then the real part of $s$ equals $1 / 2$, and hence $u^{0}$ does not belong to $L^{2}\left(\left[y_{0}, \infty\left[, y^{-2} d y\right)\right.\right.$ unless both $A$ and $B$ equal zero. Therefore, we have the following.

Corollary 2.2. If $u$ is a Neumann eigenfunction with eigenvalue $E \geqslant \frac{1}{4}$, then the zeroth Fourier coefficient $u^{0}$ vanishes identically on $\left[y_{0}, \infty[\right.$.

In the classical spectral theory of a quotient of $\mathbb{H}$ by a lattice in $S L_{2}(\mathbb{R})$, a Laplace eigenfunction $u$ with vanishing zeroth Fourier coefficient in each cusp is called a (weight zero) Maass cusp form. Even though most of the domains that we will consider are not fundamental domains for discrete groups of isometries, we will adapt this terminology.

Definition 2.3. If $u$ is an eigenfunction for the Neumann Laplacian on a domain with a cusp, and $\left(u \cdot \mathbb{1}_{\left[y_{0}, \infty[\right.}\right)^{0} \equiv 0$, then we will call $u$ a cusp form. 
Traditionally, the Neumann eigenfunctions for $\mathcal{T}_{\cos (\pi / n)}$ are called even cusp forms whereas the solutions to the Dirichlet eigenvalue problem are called odd cusp forms. We will not consider odd cusp forms in this paper.

2.3. A related quadratic form. We wish to apply analytic perturbation theory to study the behavior of eigenfunctions of $\mathcal{E}$ on $\mathcal{T}_{t}$ as we vary $t$. Because the eigenvalues of $\mathcal{E}$ might lie inside the essential spectrum, standard perturbation theory does not apply directly. Following ColinDeVerdière82 and PhlSrn85, we will use a modification of $\mathcal{E}$ first constructed by P. Lax and R. Phillips [LaxPhl] LaxPhl80.4 In this section, we recall the construction, show that the eigenvalues of the modification are isolated, and relate the eigenfunctions of the modification to those of $\mathcal{E}$.

For $\beta>y_{0}$, let $Z_{\beta}$ denote the set of $u \in \mathcal{D}(\bar{\Omega})$ such that for each $y \geqslant \beta$ we have $u^{0}(y)=0$. Let $L_{\beta}^{2}(\Omega, d m)$ denote the Hilbert space completion of $Z_{\beta}$ with respect to $u \mapsto \mathcal{N}(u)^{\frac{1}{2}}$. Let $\mathcal{N}_{\beta}$ denote the restriction of $\mathcal{N}$ to $L_{\beta}^{2}(\Omega)$.

Let $H_{\beta}^{1}(\Omega)$ denote the Hilbert space completion of $Z_{\beta}$ with respect to the norm $u \mapsto(\mathcal{E}(u)+\mathcal{N}(u))^{\frac{1}{2}}$. The restriction, $\mathcal{E}_{\beta}$, of $\mathcal{E}$ to $H_{\beta}^{1}(\Omega)$ is a closed, densely defined quadratic form on $L_{\beta}^{2}(\Omega)$. A simple argument shows that

$$
L_{\beta}^{2}(\Omega)=\left\{u \in L^{2}(\Omega, d m), \mid \forall y>\beta, u^{0}(y)=0 .\right\},
$$

and

$$
H_{\beta}^{1}(\Omega)=\left\{u \in H^{1}(\Omega), \mid \forall y>\beta, u^{0}(y)=0 .\right\} .
$$

In the sequel it will be more convenient to replace $Z_{\beta}$ by the following other set.

Definition 2.4. Define $W_{\beta}$ to be the set of functions $u$ in $H_{\beta}^{1}$ such that

- $u$ extends to a continuous function on the closure $\bar{\Omega}$ of $\Omega$,

- $u$ is smooth on $\Omega \backslash\{y=\beta\}$.

Observe that since $Z_{\beta} \subset W_{\beta}$, the closure of $W_{\beta}$ with respect to the norm $u \mapsto$ $(\mathcal{E}(u)+\mathcal{N}(u))^{\frac{1}{2}}$ is $H_{\beta}^{1}$. The latter assertion says that $W_{\beta}$ is a core of the quadratic form $\mathcal{E}_{\beta}$

Let $\Delta_{\beta}$ denote the unique operator such that $\operatorname{dom}\left(\Delta_{\beta}\right) \subset H_{\beta}^{1}$ and that satisfies $\mathcal{N}_{\beta}\left(\Delta_{\beta} u, v\right)=\mathcal{E}_{\beta}(u, v)$ for each $u \in \operatorname{dom}\left(\Delta_{\beta}\right), v \in H_{\beta}^{1}(\Omega)$.

Lemma 2.5 ([LaxPhl $)$. For each $\beta>y_{0}$, the resolvent of $\Delta_{\beta}$ is compact. Hence, the spectrum of $\mathcal{E}_{\beta}$ with respect to $\mathcal{N}_{\beta}$ is discrete and each eigenspace is finite dimensional.

Proof. Using the Fourier decomposition, one shows that for each $b>0$, the set of $v \in H_{\beta}^{1}(\Omega)$ such that $\mathcal{N}(v) \leqslant 1$ and $\mathcal{E}(v) \leqslant b$ is compact in $L_{\beta}^{2}(\Omega)$ (see Lemma 8.7 LaxPhl]). It follows that $\Delta_{\beta}$ has compact resolvent. Hence, by standard spectral theory, the spectrum is discrete and the eigenspaces are finite dimensional.

Definition 2.6 (cusp form). We will say that an eigenfunction $u$ of $\mathcal{E}_{\beta}$ with respect to $\mathcal{N}_{\beta}$ is a cusp form if and only for each $y>y_{0}$ we have $u^{0}(y)=0$.

Lemma 2.7. The following assertions are equivalent:

(1) $u$ is a cusp form of $\mathcal{E}$ with respect to $\mathcal{N}$.

\footnotetext{
${ }^{4}$ See page 206 of [LaxPhl] under the heading 'A related quadratic form'.
} 
(2) There exists $\beta>y_{0}$ such that $u$ is a cusp form of $\mathcal{E}_{\beta}$ with respect to $\mathcal{N}_{\beta}$.

(3) For each $\beta>y_{0}$, the function $u$ is a cusp form for $\mathcal{E}_{\beta}$ with respect to $\mathcal{N}_{\beta}$.

Proof. (1) $\Rightarrow(2)$ : If $u$ is an eigenfunction of $\mathcal{E}$ with eigenvalue $E$, then by Lemma 2.1 the zeroth Fourier coefficient $u^{0}$ satisfies the differential equation $0=\left(u^{0}\right)^{\prime \prime}+$ $\left(E / y^{2}\right) \cdot u^{0}$. Since $u^{0}(y)$ vanishes for $y>y_{0}$, it must vanish for $y>\beta$.

$(2) \Rightarrow(1)$ : Fix a smooth function $\chi$ such that $\chi(y)=0$ for $y \leqslant \frac{2 y_{0}+\beta}{3}$ and $\chi(y)=1$ for $y \geqslant \frac{y_{0}+2 \beta}{3}$. If $u^{0}(y)=0$ for each $y>y_{0}$, then

$$
\mathcal{N}_{\beta}\left(u, v-\chi \cdot v^{0}\right)=\mathcal{N}(u, v),
$$

and

$$
\mathcal{E}(u, v)=\mathcal{E}\left(u, v-\chi \cdot v^{0}\right)=\mathcal{E}_{\beta}\left(u, v-\chi \cdot v^{0}\right),
$$

for each $v \in H^{1}(\Omega)$. Thus, if $\mathcal{E}_{\beta}(u, v)=E \cdot \mathcal{N}_{\beta}(u, v)$, then $\mathcal{E}(u, v)=E \cdot \mathcal{N}(u, v)$.

$(2) \Leftrightarrow(3)$ : Follows from the equivalence of (1) and (2).

Not every eigenfunction $u$ of $\mathcal{E}_{\beta}$ is an eigenfunction of $\mathcal{E}$. For example, if $w=$ $\cos (\pi / n)$ and $E_{s}$ is an Eisenstein series whose zeroth Fourier coefficient vanishes at $y=\beta$, then $E_{s}(x, y)-E_{s}^{0}(y) \cdot \chi_{[\beta, \infty[}(y)$ is an eigenfunction of $\mathcal{E}_{\beta}$. This function is not smooth across $y=\beta$, but elliptic regularity implies that each eigenfunction of $\mathcal{E}$ is smooth.

\section{REAL-ANALYTICITY AND GENERIC PROPERTIES OF EIGENFUNCTIONS}

Let $S=[0,1] \times\left[y_{0}, \infty\right)$ and let $\beta>\underline{\alpha}>y_{0}$. In this section, we consider a fixed domain $\Omega$ that contains the cusp $S$ and a real-analytic family $t \mapsto q_{t}$ of quadratic forms defined on $H_{\beta}^{1}(\Omega) \subset L_{\beta}^{2}(S, d m)$ that represents the cusp of width $w_{t}$ for $y>y_{0}$ and some real-analytic function $t \mapsto w_{t}$ (see Definition 3.1 below). We prove the following dichotomy: Either there exists a real-analytic eigenfunction branch consisting of 'cusp forms' or the set of $t$ such that $q_{t}$ has a 'cusp form' eigenfunction is countable. This fact is fundamental to the proofs of both Theorem 1.1 and Theorem 1.2 .

Let $S=[0,1] \times\left[y_{0}, \infty\left[\right.\right.$ and let $\beta>\underline{\alpha}>y_{0}$. For any $w>0$, we can define a transformation $\widehat{\Phi}_{w}$ between $L_{\beta}^{2}(S)$ and $L_{\beta}^{2}\left(S_{w, y_{0}}\right)$ by asking that, for any $u \in L_{\beta}^{2}(S)$ the function $v:=\widehat{\Phi}_{w}(u)$ is defined by $v(x, y)=\frac{1}{\sqrt{w}} u\left(\frac{x}{w}, y\right)$. Since, in the sequel $y_{0}$ will be fixed, we will drop the index $y_{0}$ and denote by $S_{w}$ the cusp of width $w$.

It is straighforward that $\widehat{\Phi}_{w}$ is an isometry between $L_{\beta}^{2}(S)$ and $L_{\beta}^{2}\left(S_{w}\right)$. Moreover $\widehat{\Phi}_{w}$ also preserves $H_{\beta}^{1}$ in the sense that $\widehat{\Phi}_{w}(u) \in H_{\beta}^{1}\left(S_{w}\right)$ if and only if $u \in H_{\beta}^{1}(S)$.

We may thus define $\mathcal{E}_{\beta, w}$ the quadratic form obtained by pulling-back $\mathcal{E}_{\beta}$ on $S_{w}$ using $\widehat{\Phi}_{w}$. This quadratic form is then closed on the domain $H_{\beta}^{1}(S)$, and for each $u \in H_{\beta}^{1}(S)$

$$
\mathcal{E}_{\beta, w}(u)=\int_{S} w^{-2} \cdot\left|\partial_{x} u(x, y)\right|^{2}+\left|\partial_{y} u(x, y)\right|^{2} d x d y .
$$

Definition 3.1. Let $\Omega$ be a domain that has $S$ as a cusp and $\beta>\underline{\alpha}>y_{0}$. Let $q$ be a quadratic form closed over the domain $H_{\beta}^{1}(\Omega)$. We will say that $q$ represents the cusp of width $w$ for $y \geqslant \underline{\alpha}$ if, for any $u \in H_{\beta}^{1}$ that is supported in $\{y>\underline{\alpha}\}$ we have

$$
q(u)=\mathcal{E}_{\beta, w}(u) .
$$


For such a quadratic form, we will say that an eigenfunction $u$ is a cuspform if $u^{0}(y)$ vanishes on $\left\{y_{0} \leqslant y \leqslant \beta\right\}$.

The aim of this section is to prove that being a cuspform is a real-analytic condition. To make this statement precise we have to consider a family $q_{t}$ of quadratic forms that satisfies the following assumptions.

Assumption 3.2. Let $t_{-}\left\langle t_{+}\right.$and $\beta>\underline{\alpha}>y_{0}$. For each $\left.t \in I:=\right] t_{-}, t_{+}[$, let $w_{t}$ be a positive real-analytic function on $I$. Let $q_{t}$ denote a nonnegative, closed quadratic form with domain $H_{\beta}^{1}(S)$ that represents the cusp of width $w_{t}$ for $y \geqslant \underline{\alpha}$.

Lastly, we assume that the family $t \mapsto q_{t}$ is real-analytic of type (a) in the sense of [Kato. That is, for each $u \in H_{\beta}^{1}(S)$, the map $t \mapsto q_{t}(u)$ is real-analytic.

A straightforward application of analytic perturbation theory- Kato §VIIgives the following.

Theorem 3.3 (Existence of a real-analytic eigenbasis). Let $t \mapsto q_{t}$ satisfy the assumptions above 3.2. Then there exist a collection of real-analytic paths $\{t \mapsto$ $\left.u_{j, t} \in L^{2}(\Omega, d m) \mid j \in \mathbb{Z}^{+}\right\}$and a collection of real-analytic functions $\left\{t \mapsto \lambda_{j, t} \in\right.$ $\left.\mathbb{R} \mid j \in \mathbb{Z}^{+}\right\}$so that for each $t$, the set $\left\{u_{j, t} \mid j \in \mathbb{Z}\right\}$ is an orthonormal basis for $L_{\beta}^{2}(\Omega, d m)$, and for each $(j, t)$, the function $u_{j, t}$ is an eigenfunction of $q_{t}$ with eigenvalue $\lambda_{j, t}$.

Proof. Since the embedding from $H_{\beta}^{1}$ into $L_{\beta}^{2}$ is compact, for any $t$ the spectrum of $q_{t}$ consists only in eigenvalues. The proof is then similar to the proof of Theorem 3.9 in Kato] §VIII.3.5.

For $u \in W_{\beta}$, define

$$
L(u)=\lim _{y \rightarrow \beta^{-}} \frac{u^{0}(y)}{y-\beta} .
$$

Lemma 3.4. An eigenfunction $u$ of $q_{t}$ is a cusp form if and only if $L(u)=0$.

Proof. $L(u)$ is the left-sided derivative of $u^{0}$ at $\beta$. Since $u$ is an eigenfunction, $u \in W_{\beta}$ and $u^{0}$ is a solution to a second order ordinary differential equation on $\left[y_{0}, \beta\right]$ with $u_{0}(\beta)=0$. Thus, $u^{0}$ vanishes identically on $\left[y_{0}, \beta\right]$ if and only if $L(u)=0$.

For real-analytic eigenbranches we have the following.

Lemma 3.5. Let $\left(u_{t}, \lambda_{t}\right)$ be an analytic eigenbranch of $q_{t}$ then the mapping $t \mapsto$ $L\left(u_{t}\right)$ is analytic on $] t_{-}, t_{+}[$.

Proof. The zeroth mode $u_{t}^{0}$ of $u_{t}$ is a solution to the ODE

$$
-u^{\prime \prime}-\frac{\lambda_{t}}{y^{2}} \cdot u=0
$$

on $[\underline{\alpha}, \beta]$ with Dirichlet boundary condition at $\beta$. Denote by $G_{\lambda}$ the unique solution to this ordinary differential equation that satisfies $G_{\lambda}(\beta)=0, G_{\lambda}^{\prime}(\beta)=1$. Since the coefficients of the ordinary differential equation depend analytically on the parameter $\lambda$, the mapping $\lambda \mapsto G_{\lambda}$ is analytic (with values in $\mathcal{C}^{2}([\underline{\alpha}, \beta])$ for instance). Moreover, for each compact set $K \subset] t_{-}, t_{+}$[ we can find $\underline{\alpha}<\alpha_{K}<\beta$ such that, for 
each $t \in K, \int_{\alpha_{K}}^{\beta} G_{\lambda}>0$. Since $u_{t}$ is a multiple of $G_{\lambda_{t}}$, we then have

$$
\begin{aligned}
L\left(u_{t}\right) & =\left(\int_{\alpha_{K}}^{\beta} G_{\lambda_{t}}(y) d y\right)^{-1} \cdot \int_{\alpha_{K}}^{\beta} u_{t}^{0}(y) d y \\
& =\left(\int_{\alpha_{K}}^{\beta} G_{\lambda_{t}}(y) d y\right)^{-1} \cdot \int_{0}^{1} \int_{\alpha_{K}}^{\beta} u_{t}(x, y) d x d y,
\end{aligned}
$$

Analyticity on $K$ then follows from the analyticity of $t \mapsto u_{t}$ and $t \mapsto G_{\lambda_{t}}$ and the choice of $\alpha_{K}$.

We will say that real-analytic eigenfunction branch $u_{t}$ of $q_{t}$ is a cusp form eigenbranch if and only if for each $t \in I$, the eigenfunction $u_{t}$ is a cusp form (see Definition 3.1). Using the real-analyticity proved in Lemma 3.4 we obtain the following

Corollary 3.6. If $u_{t}$ is a real-analytic eigenfunction branch that is not a cusp form eigenbranch, then the set of $t \in I$ such that $u_{t}$ is a cusp form is discrete.

We now proceed to prove that if $q_{t}$ has no real-analytic cusp form eigenbranch then, for a generic $t$, the form $q_{t}$ has no cusp form. As it turns out, we will actually first prove that the spectrum of $q_{t}$ is generically simple.

Let $I_{\text {mult }}$ denote the set of $t \in I$ such that $q_{t}$ has an eigenspace of dimension at least two.

Proposition 3.7. If $q_{t}$ does not have a real-analytic cusp form eigenbranch, then $I_{\text {mult }}$ is countable.

Proof. Let $\left\{u_{j, t} \mid j \in \mathbb{Z}^{+}, t \in I\right\}$ and $\left\{\lambda_{j, t} \mid j \in \mathbb{Z}^{+}, t \in I\right\}$ be as in Theorem 3.3. For each $j, k \in \mathbb{Z}^{+}$, let $Z_{j, k}=\left\{t \mid \lambda_{j, t}=\lambda_{k, t}\right\}$. Since each eigenspace of $q_{t}$ is spanned by a finite collection of $\left\{u_{j, t}\right\}$, the union $\bigcup_{j, k} Z_{j, k}$ equals $I_{\text {mult }}$.

The function $t \mapsto \lambda_{j, t}-\lambda_{k, t}$ is analytic, and hence $Z_{j, k}=\left\{t \mid \lambda_{j, t}=\lambda_{k, t}\right\}$ is either countable or equals $I$. Thus to prove the claim, it suffices to show that it is not possible for $Z_{j, k}$ to equal $I$.

Suppose that there exists $j$ and $k$ so that $u_{j, t}$ and $u_{k, t}$ are real-analytic eigenbranches so that $\lambda_{j, t}=\lambda_{k, t}$ for each $t \in I$. To prove the proposition, it suffices to produce a linear combination $u_{t}$ of $u_{j, t}$ and $u_{k, t}$ so that for each $t \in I$, the function $u_{t}$ is a real-analytic cusp form eigenbranch.

By hypothesis, neither $u_{j, t}$ nor $u_{k, t}$ are cusp form eigenbranches. By Corollary 3.6. the set $J$ of $t$ such that either $u_{j, t}$ or $u_{k, t}$ is a cusp form is discrete.

For each $t \notin J$, define

$$
u_{t}=\frac{L\left(u_{k, t}\right) \cdot u_{j, t}-L\left(u_{j, t}\right) \cdot u_{k, t}}{\sqrt{L\left(u_{j, t}\right)^{2}+L\left(u_{k, t}\right)^{2}}} .
$$

It suffices to show that $t \rightarrow u_{t}$ extends to a real-analytic function on $I$. Indeed, since $L$ is linear, we have $L\left(u_{t}\right)=0$ for each $t \notin J$. By Corollary 3.5, the real-analytic extension would satisfy $L\left(u_{t}\right) \equiv 0$.

The order of vanishing of $t \mapsto L\left(u_{j, t}\right)$ (resp. $t \mapsto L\left(u_{k, t}\right)$ ) is finite at each $t \in J$. If the order of vanishing of $L\left(u_{k, t}\right)$ at $t_{0} \in J$ is at least the order of vanishing of $L\left(u_{j, t}\right)$ at $t_{0}$, then the ratio $L\left(u_{k, t}\right) / L\left(u_{j, t}\right)$ has a real-analytic extension near $t_{0}$. 
Hence, factorizing $L\left(u_{j, t}\right)$ we obtain that

$$
\begin{aligned}
& \frac{L\left(u_{k, t}\right)}{\sqrt{L\left(u_{j, t}\right)^{2}+L\left(u_{k, t}\right)^{2}}}=\frac{L\left(u_{k, t}\right)}{L\left(u_{j, t}\right)} \cdot\left(1+\left(\frac{L\left(u_{k, t}\right)}{L\left(u_{j, t}\right)}\right)^{2}\right)^{-\frac{1}{2}} \text {, and } \\
& \frac{L\left(u_{j, t}\right)}{\sqrt{L\left(u_{j, t}\right)^{2}+L\left(u_{k, t}\right)^{2}}}=\left(1+\left(\frac{L\left(u_{k, t}\right)}{L\left(u_{j, t}\right)}\right)^{2}\right)^{-\frac{1}{2}}
\end{aligned}
$$

have real-analytic extensions near $t_{0}$. If the order of vanishing of $L\left(u_{k, t}\right)$ at $t_{0}$ is at most the order of vanishing of $L\left(u_{j, t}\right)$ at $t_{0}$, then a similar argument applies by factorizing $L\left(u_{k, t}\right)$ everywhere. Thus, $u_{t}$ extends to a real-analytic cusp form eigenbranch.

Let $I_{\text {cf }}$ denote the set of $t \in I$ such that $q_{t}$ has at least one cusp form eigenfunction.

Proposition 3.8. If $q_{t}$ has no real-analytic cusp form eigenbranch, then $I_{\mathrm{cf}}$ is countable.

Remark 3.9 (Dichotomy). If there exists a cusp form eigenbranch, then $I_{\mathrm{cf}}=I$. Therefore, we have the following dichotomy: Either the set $I_{\mathrm{cf}}$ countable or the family $t \mapsto q_{t}$ has a real-analytic cusp form eigenbranch.

Proof of Proposition 3.8. Let $\left\{u_{j, t} \mid j \in \mathbb{Z}, t \in I\right\}$ be as in Theorem 3.3. By Corollary 3.6. the zero set $Z_{j}=\left\{t \mid L\left(u_{j, t}=0\right)\right\}$ is countable.

If each eigenspace $E$ of $q_{t}$ is one-dimensional, then there exists a unique $j$ such that $E$ equals the span of $u_{j, t}$. Thus, if $t$ does not belong to $I_{\text {mult }}$ or to any $Z_{j}$, then $t$ does not belong to $I_{\mathrm{cf}}$. In other words, $I_{\mathrm{cf}} \subset\left(\bigcup Z_{j}\right) \cup I_{\text {mult }}$. By Proposition 3.7. the set $I_{\text {mult }}$ is countable, and hence so is $I_{\mathrm{cf}}$.

\section{Perturbation theory FOR hyperbolic triangles With ONE CUSP}

In this section we use the results of the previous section to explain how Theorem 1.2 can be deduced from the existence of a triangle with a cusp that has no nonconstant Neumann eigenfunctions. This fact might be considered to be folklore as it follows from the general philosophy of using analyticity to prove generic spectral results (see HlrJdg09). The main task here is to construct a real-analytic family of quadratic forms that is associated with each real-analytic path in the moduli space of triangles.

4.1. The moduli space of triangles. First, we discuss the parametrization of the set of triangles with one cusp. The statement of Theorem 1.2 makes use of the fact that hyperbolic triangles with one cusp are parametrized by the two nonzero vertex angles. But in order to prove Theorem 1.2 , it will be more convenient to use an alternate set of parameters.

For each geodesic triangle $T$ in the hyperbolic upper half plane $\mathbb{H}^{2}$ having (exactly) one cusp, there exists a unique $c \in] 0,1[$ and $w \in[2 c, 1+c[$ so that $T$ is isometric to the domain

$$
\mathcal{T}_{c, w}=\left\{(x, y) \mid 0 \leqslant x \leqslant w, \quad(x-c)^{2}+y^{2}>1\right\} .
$$

See Figure 2 


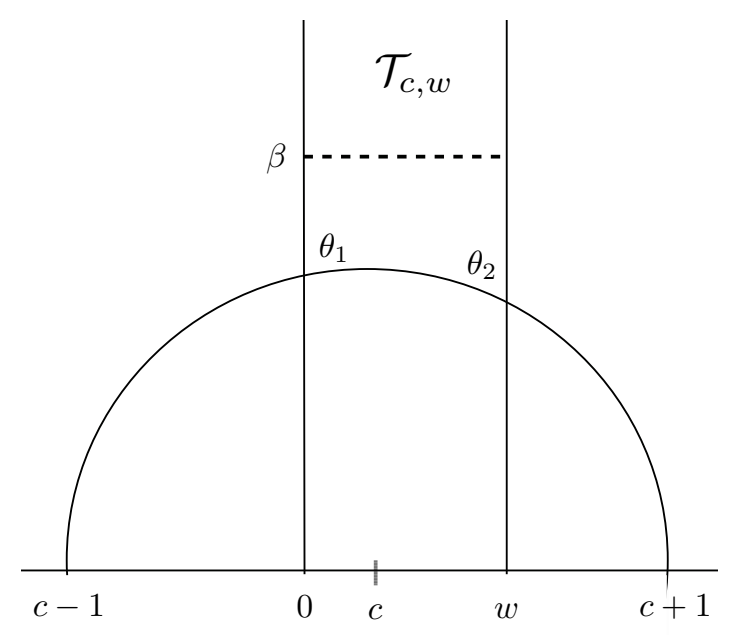

Figure 2. The triangle $\mathcal{T}_{c, w}$ in the upper half plane.

In this way, the set of hyperbolic triangles may be identified with the Euclidean triangle

$$
\mathcal{M}=\{(c, w) \mid 0 \leqslant c<1,2 c<w<c+1\} .
$$

We say that a subset of the set of triangles has measure zero if and only if the corresponding subset of $\mathcal{M}$ has measure zero. Similarly, a subset of the set of triangles is said to be a real-analytic curve if and only if the corresponding subset of $\mathcal{M}$ is a real-analytic curve.

These notions are equivalent to those used in the statement of Theorem 1.2 because the relationship between the angles $\left(\theta_{1}, \theta_{2}\right)$ and the parameters $(c, w)$ is real-analytic. Indeed, we have $c=\cos \left(\theta_{1}\right)$ and $\cos \left(\theta_{2}\right)=w-c$. See Figure 2 .

To prove Theorem 1.2 , we will apply perturbation theory. The following fact makes this approach feasible.

Proposition 4.1. Each nonconstant Neumann eigenfunction on $\mathcal{T}_{c, w}$ is a cusp form and hence an eigenfunction of the modified quadratic form $\mathcal{E}_{\beta}$.

Proof. The eigenvalue of a nonconstant Neumann eigenfunction on $T$ is at least $1 / 4$ Jdg07 $5^{5}$ Thus, the claim follows from Corollary 2.2 and Lemma 2.5.

Let $\mathcal{M}_{\mathrm{cf}}$ denote the set of $(c, w) \in \mathcal{M}$ such that there exists $\beta>1$ so that the modified quadratic from $\mathcal{E}_{\beta}$ has a cusp form. To prove Theorem 1.2 it will suffice to show that $\mathcal{M}_{\mathrm{cf}}$ has measure zero and is a countable collection of nowhere dense sets.

4.2. A family of diffeomorphisms. To show that $\mathcal{M}_{\mathrm{cf}}$ is non-generic, we will use analytic perturbation theory and Proposition 3.8. In order to use analytic perturbation theory we will have to normalize the Hilbert space and the domains of the quadratic forms. To accomplish this, we let $S=[0,1] \times[1, \infty[$ and for each $(c, w)$ we define a $C^{1}$ diffeomorphism $\varphi_{c, w}: \mathcal{T}_{c, w} \rightarrow S$ such that ${ }^{6}$

\footnotetext{
${ }^{5}$ See also Sarnak for the case of triangles that are fundamental domains for the Hecke groups.

${ }^{6}$ In HlrJdg11, we considered a simpler mapping from $\mathcal{T}_{0, t}$ onto $S$. The mapping that we define here is more complicated because it must preserve the notion of zeroth Fourier coefficient
} 
(1) The restriction of $\varphi_{c, w}$ is the identity for $y>\underline{\alpha}=(\beta+1) / 2$.

(2) For each path $\gamma: I \rightarrow \mathcal{M}$, the family $t \mapsto \varphi_{c, w}$ is a real-analytic path.

To construct $\varphi_{c, w}$, we use the fact that the map $(x, y) \mapsto x$ defines a fibration of $\mathcal{T}_{c, w}$ over $[0, w]$ and a fibration of $S$ over $[0,1]$. We define $\varphi_{c, w}$ by sending the fiber over $\{x\}$ onto the fiber over $\{x / w\}$.

Lemma 4.2. For each $\alpha \in] 0, \underline{\alpha}$, there exists a unique cubic polynomial $B_{\alpha}$ so that

- $B_{\alpha}(\alpha)=1$,

- $B_{\alpha}^{\prime}(0)=\alpha$.

- $B_{\alpha}(\underline{\alpha})=\underline{\alpha}$,

- $B_{\alpha}^{\prime}(\underline{\alpha})=1$

The coefficients of $B_{\alpha}$ are real analytic for $\left.\alpha \in\right] 0, \underline{\alpha}[$.

Moreover if $\underline{\alpha}>2+\sqrt{3}$ then, for all $\alpha \in(0,1]$, and $y \in[0, \underline{\alpha}]$, we have $B_{\alpha}^{\prime}(y)>0$.

Proof. Since $\underline{\alpha} \neq 0$, satisfying the two conditions on $B_{\alpha}^{\prime}$ is equivalent to the existence of some $A$ such that

$$
B_{\alpha}^{\prime}(y)=A \cdot y(\underline{\alpha}-y)+\frac{1}{\underline{\alpha}} \cdot y+\frac{\alpha}{\alpha} \cdot(\underline{\alpha}-y) .
$$

Denote by $Q_{\alpha}$ the cubic polynomial defined by

$$
Q_{\alpha}(y)=\int_{\alpha}^{y} z(\underline{\alpha}-z) d z .
$$

By integration, there exists some $C$ such that

$$
B_{\alpha}(y)=A \cdot Q_{\alpha}(y)+\frac{1}{\underline{\alpha}} \cdot \frac{y^{2}}{2}-\frac{\alpha}{\underline{\alpha}} \cdot \frac{(\underline{\alpha}-y)^{2}}{2}+C .
$$

Evaluating at $\alpha$ and using the condition on $B_{\alpha}(\alpha)$ we find

$$
C=1-\frac{\alpha^{2}}{2 \underline{\alpha}}-\frac{\alpha(\underline{\alpha}-\alpha)^{2}}{2 \underline{\alpha}} .
$$

Observe that $Q_{\alpha}(\underline{\alpha}) \neq 0$ if $\alpha \in[0, \underline{\alpha}$ [ and, under this condition, we can solve the last equation on $B_{\alpha}$ to find $A$. We obtain

$$
\begin{aligned}
Q(\underline{\alpha}) A & =\underline{\alpha}-\frac{\underline{\alpha}}{2}-C \\
& =\frac{1}{2 \underline{\alpha}}\left[\underline{\alpha}^{2}+\alpha^{2}-\alpha(\underline{\alpha}-\alpha)^{2}-2 \underline{\alpha}\right] \\
& =\frac{1-\alpha}{2 \underline{\alpha}}\left[\alpha^{2}+\underline{\alpha}^{2}-2 \underline{\alpha}(\alpha+1)\right], \\
& =\frac{(1-\alpha)\left[\alpha^{2}-2 \underline{\alpha} \alpha+\underline{\alpha}^{2}-2 \underline{\alpha}\right]}{2 \underline{\alpha}}
\end{aligned}
$$

It follows that we have a unique solution provided $\underline{\alpha} \neq 0$ and $0<\alpha<\underline{\alpha}$, and that the coefficients are real-analytic in $\alpha$.

We now check the last statement. For $\alpha=1$, we have $B_{\alpha}(y)=y$ so that the claim follows. For $\alpha<1$ we observe that the numerator of $A$ is a cubic polynomial

for all $y$ above some point. In particular, the vertical displacement of vertical lines should not depend on $x$ for large $y$. In HlrJdg11, we considered Dirichlet boundary conditions, and in that context there is no need to truncate the zeroth Fourier coefficient. 
that has three roots at $1, \underline{\alpha} \pm \sqrt{2 \underline{\alpha}}$. Thus, if $\underline{\alpha}>2+\sqrt{3}$ then 1 is the smallest root. Since this cubic polynomial is positive for large negative $\alpha$ and the denominator also is positive, it follows that $A$ is positive for $0<\alpha<1$. So $B_{\alpha}^{\prime}$ is a concave function, and by construction $B_{\alpha}^{\prime}(0)>0$ and $B_{\alpha}^{\prime}(\underline{\alpha})>0$. The claim follows.

Notation. We will use the notation $B_{\alpha}(y)$ as well as the notation $B(\alpha, y)$.

Define $F_{c, x}: \mathbb{R} \rightarrow \mathbb{R}$ by

$$
F_{c}(x, y)=\left\{\begin{array}{cc}
B\left(f_{c}(x), y\right) & \text { if } y \leqslant \underline{\alpha} \\
y & \text { if } y \geqslant \underline{\alpha} .
\end{array}\right.
$$

where

$$
f_{c}(x)=\sqrt{1-(x-c)^{2}}
$$

Define $\varphi_{c, w}: \mathcal{T}_{c, w} \rightarrow S$ by

$$
\varphi_{c, w}(x, y)=\left(x / w, F_{c}(x, y)\right) .
$$

Observe that the conditions on $B$ imply that $F, \partial_{x} F_{c}$ and $\partial_{y} F_{c}$ are continuous on $\mathcal{T}_{c, w}$ so that $\varphi_{c, w}$ is $C^{1}$.

We will use this function $\varphi_{c, w}$ to normalize the triangle $\mathcal{T}_{c, w}$. This is made possible by the following lemma.

Lemma 4.3. Suppose that $\partial_{y} B\left(f_{c}(x), y\right)>0$ for each $(x, y) \in \mathcal{T}_{c, w} \cap\{y \leqslant \underline{\alpha}\}$ then the map $\varphi_{c, w}$ is a $C^{1}$ diffeomorphism from $\mathcal{T}_{c, w}$ onto $S$.

In particular, for each $\underline{\alpha}>2+\sqrt{3}$ and each $(c, w) \in \mathcal{M}$, the mapping $\varphi_{c, w}$ is a $C^{1}$ diffeomorphism from $\mathcal{T}_{c, w}$ onto $S$.

Proof. It suffices to show that the map $F_{c, x}$ is a $C^{1}$ diffeomorphism from $\left[f_{c}(x), \infty[\right.$ onto $\left[1, \infty\left[\right.\right.$. By assumption $\partial_{y} B\left(f_{c}(x), y\right)>0$ for each $x$. We have $B\left(f_{c}(x), f_{c}(x)\right)=$ $1, B\left(f_{c}(x), \underline{\alpha}\right)=\underline{\alpha}$ and $\partial_{y} B\left(f_{c}(x), \underline{\alpha}\right)=1$. Since $F_{c, x}$ is the identity for $y>\underline{\alpha}$, we find that $F_{c, x}$ is a $C^{1}$ diffeomorphism from $\left[f_{c}(x), \infty[\right.$ onto $[1, \infty]$.

For each $\underline{\alpha}$ and each $M \subset \mathcal{M}$, we define $X_{\underline{\alpha}, M}$ and $A_{\underline{\alpha}, M}$ by

$$
\begin{gathered}
X_{\underline{\alpha}, M}:=\left\{(x, y, c, w) \mid(c, w) \in M,(x, y) \in \mathcal{T}_{c, w}, y \leqslant \underline{\alpha}\right\}, \\
A_{\underline{\alpha}, M}:=\{(a, b, c, w) \mid(c, w) \in M,(a, b) \in S, b \leqslant \underline{\alpha}\} .
\end{gathered}
$$

We then have

Lemma 4.4. For each $\underline{\alpha}, M$, each of the following maps is analytic on $X_{\underline{\alpha}, M}$ :

(1) $(x, y, c, w) \mapsto \varphi_{c, w}(x, y)$

(2) $(x, y, c, w) \mapsto \partial_{x} \varphi_{c, w}(x, y)$

(3) $(x, y, c, w) \mapsto \partial_{y} \varphi_{c, w}(x, y)$

If, for each $(c, w) \in M$, the assumption of Lemma 4.3 holds then the map $(a, b, c, w) \mapsto$ $\varphi_{c, w}^{-1}(a, b)$ is also analytic on $A_{\underline{\alpha}, M}$.

Moreover, each restriction extends analytically to an open neighbourhood.

Proof. The coefficients of the cubic polynomial $B_{\alpha}$ depend analytically on $\alpha$ and hence $(\alpha, y) \mapsto B(\alpha, y)$ is analytic. The map $(c, x) \mapsto f_{c}(x)$ is analytic and hence it follows that map (1) is analytic. Maps (2) and (3) are therefore analytic.

Since $(\alpha, y) \mapsto B(\alpha, y)$ is analytic and $\partial_{y} B(\alpha, y)>0$ for $y>0$, the implicit function theorem (Theorem 2.1.2 in Hörm] implies that there exists a function $(\alpha, b) \mapsto Y_{\alpha}(b)$ which is analytic and a solution to 


$$
B_{\alpha}\left(Y_{\alpha}(b)\right)-b=0
$$

We then have

$$
\varphi_{c, w}^{-1}(a, b)=\left(w \cdot a, Y_{f_{c}(w \cdot a)}(b)\right),
$$

and, since $(c, x) \mapsto f_{c}(x)$ is analytic, the claim follows.

In the rest of the section, $\underline{\alpha}>2+\sqrt{3}$ will be fixed so that we can use lemmas 4.3 and 4.4 .

4.3. The quadratic form with fixed domain. We use the family of diffeomorphisms $\varphi_{c, w}$ to define a quadratic form $q_{t}$ with domain $H_{\beta}^{1}(S) \subset L_{\beta}^{2}(S)$ that is unitarily equivalent to $\mathcal{E}_{\beta}$ on $H_{\beta}^{1}\left(\mathcal{T}_{c, w}\right) \subset L_{\beta}^{2}\left(\mathcal{T}_{c, w}\right)$.

Define $\Phi_{c, w}: L^{2}\left(S, d a d b / b^{2}\right) \rightarrow L^{2}\left(\mathcal{T}_{c, w}, d x d y / y^{2}\right)$ by

$$
\Phi_{c, w}(u)=y \cdot \sqrt{\left|\operatorname{det}\left(\operatorname{Jac}\left(\varphi_{c, w}\right)\right)\right|} \cdot\left(\frac{u}{b} \circ \varphi_{c, w}\right)
$$

where Jac is the operator that returns the Jacobian matrix of a map.

Lemma 4.5. The isometry $\Phi_{c, w}$ is a unitary isomorphism from $L_{\beta}^{2}(S)$ onto $L_{\beta}^{2}\left(\mathcal{T}_{c, w}\right)$ and it maps $H_{\beta}^{1}(S)$ onto $H_{\beta}^{1}\left(\mathcal{T}_{c, w}\right)$.

On functions that are supported in $b \geqslant \underline{\alpha}, \Phi_{c, w}$ coincides with $\widehat{\Phi}_{w}$.

Proof. We have

$$
\begin{aligned}
\int_{\mathcal{T}_{c, w}}\left|\Phi_{c, w}(u)\right|^{2} \frac{d x d y}{y^{2}} & =\int_{\mathcal{T}_{c, w}}\left(\frac{u}{b} \circ \varphi_{c, w}\right)^{2}\left|\operatorname{det}\left(\operatorname{Jac}\left(\varphi_{c, w}\right)\right)\right| \cdot y^{2} \cdot \frac{d x d y}{y^{2}} \\
& =\int_{S}\left(\frac{u}{b}\right)^{2} d a d b .
\end{aligned}
$$

It follows that $\Phi_{c, w}$ is a unitary isomorphism from $L_{\beta}^{2}(S)$ onto $L_{\beta}^{2}\left(\mathcal{T}_{c, w}\right)$.

Let $u \in H_{\beta}^{1}(S)$. Since $\varphi_{c, w}$ is a $\mathcal{C}^{1}$ diffeomorphism and $\sqrt{\left|\operatorname{det}\left(\operatorname{Jac}\left(\varphi_{c, w}\right)\right)\right|}$ is continuous on $\mathcal{T}_{c, w}$ and smooth away from $y=\beta$, then $\Phi_{c, w}(u)$ is continuous and in $H_{\beta}^{1}\left(\mathcal{T}_{c, w} \backslash\{y=\beta\}\right)$. The jump formula implies that $\Phi_{c, w}(u) \in H_{\beta}^{1}\left(\mathcal{T}_{c, w}\right)$.

Since, for $y>\underline{\alpha}, \varphi_{c, w}(x, y)=\left(\frac{x}{w}, y\right)$, the last statement is a direct verification.

Definition 4.6. Define the quadratic form $q_{c, w}$ on $H_{\beta}^{1}(S) \subset L^{2}\left(S, d a d b / b^{2}\right)$ by

$$
q_{c, w}(u):=\mathcal{E}_{\beta} \circ \Phi_{c, w}(u) .
$$

Lemma 4.7. $u$ is a cusp form for $q_{c, w}$ if and only if $v=\Phi_{c, w} \circ u$ is a cusp form for $\mathcal{E}$ on $\mathcal{T}_{c, w}$.

Proof. If $y \geqslant \underline{\alpha}$, then $\varphi_{c, w}(x, y)=(x / w, y)$. It follows that if $y \geqslant \underline{\alpha}$, then $u^{0}(y)=0$ if and only if $v^{0}(y)=0$. For $y \geqslant 1$, the function $v^{0}$ is a solution to a second order ordinary differential equation, and hence $v^{0}(y)=0$ for $y \geqslant \underline{\alpha}$ if and only if $v^{0}(y)=0$ for $y \geqslant 1$.

It will be convenient to have the following alternate form for $q_{t}$.

Proposition 4.8. We have

$$
q_{c, w}(u)=\int_{S} \nabla\left(\rho_{c, w} \cdot u\right) \cdot Q_{c, w} \cdot{\overline{\nabla\left(\rho_{c, w} \cdot u\right)}}^{*} d a d b
$$


where $\rho_{c, w}: S \mapsto \mathbb{R}$ is defined by

$$
\rho_{c, w}=\frac{\left(y \cdot \sqrt{\mid \operatorname{det}\left(\operatorname{Jac}\left(\varphi_{c, w}\right) \mid\right.}\right) \circ \varphi_{c, w}^{-1}}{b},
$$

and $Q_{c, w}: S \rightarrow G L_{2}(\mathbb{R})$ is defined by

$$
Q_{c, w} \circ \varphi_{c, w}=\frac{1}{\operatorname{det}\left(\operatorname{Jac}\left(\varphi_{c, w}\right)\right)} \cdot \operatorname{Jac}\left(\varphi_{c, w}\right) \cdot \operatorname{Jac}\left(\varphi_{c, w}\right)^{*} .
$$

Moreover, $q_{c, w}$ represents the cusp of width $w$ for $y \geqslant \underline{\alpha}$.

Proof. This is a straightforward calculation using the chain rule and the change of variables formula.

4.4. Analytic paths in $\mathcal{M}$. Let $I=] t_{-}, t_{+}[$and let $\gamma: I \rightarrow \mathcal{M}$ be a real-analytic path.

Theorem 4.9. The family of quadratic forms $t \mapsto q_{\gamma(t)}$ is analytic of type (a) in the sense of [Kato.

Proof. For each $t$, the quadratic form $q_{\gamma(t)}=\mathcal{E}_{\beta} \circ \Phi_{\gamma(t)}$ is a closed form with domain $H_{\beta}^{1}(S)$. It suffices to show that for each $u \in H_{\beta}^{1}(S)$, the function $t \mapsto q_{\gamma(t)}(u)$ is real-analytic.

By Proposition 4.8, we have

$$
q_{c, w}(u)=\int_{1}^{\underline{\alpha}} \int_{0}^{1} I_{t} d a d b+\int_{\underline{\alpha}}^{\infty} \int_{0}^{1} I_{t} d a d b .
$$

where

$$
I_{t}=\nabla\left(\rho_{\gamma(t)} \cdot u\right) \cdot Q_{\gamma(t)} \cdot{\bar{\nabla}\left(\rho_{\gamma(t)} \cdot u\right)^{*}}^{*}
$$

If $(a, b) \in[0,1] \times[\underline{\alpha}, \infty[$, then the matrix $Q(a, b)$ is given by

$$
Q=\left(\begin{array}{cc}
\frac{1}{w_{t}^{2}} & 0 \\
0 & 1
\end{array}\right)
$$

and $\rho_{\gamma(t)}(a, b)=1$. Thus, the second integral on the right of 12 depends analytically on $t$.

It remains to consider the integral over $[0,1] \times[1, \underline{\alpha}]$. The integrand $I_{t}$ can be expanded into a finite sum of terms of the form

$$
\int_{1}^{\underline{\alpha}} \int_{0}^{1} w(a, b) \cdot H(t, a, b) d a d b
$$

where $H$ is a function that is obtained by multiplying $\rho$, or its derivatives and the entries of $Q$ and $w$ is one of the $L^{1}$ functions obtained by making the product $v_{1} v_{2}$ where both $v_{i}$ are either $u$ or one of its partial derivatives.

By Lemma 4.4 the coordinates of $\varphi_{c, w}$ and $\varphi_{c, w}^{-1}$ are analytic functions of $(c, w)$. It follows that $(t, a, b) \mapsto \rho_{\gamma(t)}(a, b)$ and $(t, a, b) \mapsto Q_{i j}(t)(a, b)$ are analytic (in a neighborhood of $I \times[0,1] \times[1, \underline{\alpha}])$. In all possible choices, the function $H$ then is analytic.

The analyticity of $t \mapsto q_{\gamma(t)}(u)$ follows from Lemma 4.10 below. 
Lemma 4.10. If $H: I \times[0,1] \times[1, \underline{\alpha}]$ is analytic, then for each $p \in L^{1}([0,1] \times[1, \underline{\alpha}])$, the function

$$
t \longmapsto \int_{1}^{\underline{\alpha}} \int_{0}^{1} p(a, b) \cdot H(t, a, b) d a d b
$$

is analytic on $I$.

Proof. There exists an open neighborhood $U \subset \mathbb{C}^{3}$ of $I \times[0,1] \times[1, \underline{\alpha}]$ such that the map $h$ extends to a holomorphic function on $U$. Since $[0,1] \times[1, \underline{\alpha}]$ is compact,

$$
\frac{H(t, a, b)-H(s, a, b)}{t-s}
$$

converges uniformly to $\frac{d}{d t} H(s, a, b)$ as $t$ approaches $s$. It follows that the (complex) $t$-derivative of the map in 14 exists at each $t \in U$.

4.5. Generic absence of cusp forms. Given Theorem 4.9, we now explain why the generic triangle $\mathcal{T}_{c, w}$ has no cusp forms provided that one triangle has none.

Theorem 4.11. If there exists a point $\left(c_{0}, w_{0}\right) \in \mathcal{M}$ such that $\mathcal{E}$ on $L_{\beta}^{2}\left(\mathcal{T}_{c_{0}, w_{0}}, d m\right)$ has no nonconstant eigenfunction, then $\mathcal{M}_{\mathrm{cf}}$ has measure zero and is a countable union of nowhere dense sets.

Proof. By Proposition 4.1, the quadratic form $\mathcal{E}_{\beta}$ on $L_{\beta}^{2}\left(\mathcal{T}_{c_{0}, w_{0}}, d m\right)$ has no cusp form, and hence by Lemma 4.7, the quadratic form $q_{c_{0}, w_{0}}$ has no cusp form.

To show that $\mathcal{M}_{\mathrm{cf}}$ has measure zero, we apply Fubini's theorem in a fashion similar to HlrJdg09]: Let $\gamma_{c_{0}}(t)=\left(c_{0}, w_{0}+t\right)$ and apply Lemma 3.8 to find that the set $B$ of $w$ such that $\left(c_{0}, w\right) \in \mathcal{M}_{\text {cf }}$ is countable. For each $w \notin B$, let $\gamma_{w}(s)=\left(c_{0}+\right.$ $s, w)$ and apply Lemma 3.8 to find that the intersection $I_{w}$ of the line $\{(c, w) \mid c \in \mathbb{R}\}$ with $\mathcal{M}_{\mathrm{cf}}$ is countable. Hence for each $w \notin B$, the set $I_{w}$ has measure zero with respect to the linear measure $d a$. Hence, the measure of $\mathcal{M}_{\mathrm{cf}}$ equals the measure of $\bigcup_{w \in B} I_{w}$. Since $B$ is countable, the measure equals zero.

For $N \in \mathbb{Z}$, let $\mathcal{M}_{\mathrm{cf}}^{N}$ be the set of $(c, w) \in \mathcal{M}$ such that $\mathcal{E}$ on $L^{2}\left(\mathcal{T}_{c, w}, d m\right)$ has a cusp form with eigenvalue at most $N$. Using the continuity of $(c, w) \rightarrow q_{c, w}$ and the continuity of linear functional $L$, one can show that $\mathcal{M}_{\mathrm{cf}}^{N}$ is closed. Thus, it suffices to show that $\mathcal{M}_{\mathrm{cf}}^{N}$ is nowhere dense.

Given a point $(c, w) \in \mathcal{M}_{\mathrm{cf}}^{N}$, let $\gamma:[0,1] \rightarrow \mathcal{M}$ be a real-analytic path joining $\left(c_{0}, w_{0}\right)$ to $(c, w)$. Since $\mathcal{E}_{\beta}$ on $L_{\beta}^{2}\left(\mathcal{T}_{c_{0}, w_{0}}, d m\right)$ has no cusp forms, the family $t \mapsto q_{\gamma(t)}$ has no cusp form eigenfunction branch. It follows from Lemma 3.8, that for each open neighborhood $U$ of $(c, w)$, there exists $t \in[0,1]$ such that $\gamma(t) \in U$ and $q_{\gamma(t)}$ has no cusp forms. Hence $\mathcal{M}_{\mathrm{cf}}^{N}$ is nowhere dense.

\section{The FAMILY $\mathcal{T}_{t}$}

In the remainder of this paper we consider the specific family of triangles $\mathcal{T}_{t}=\mathcal{T}_{0, t}$ defined in the introduction. In particular, we will study the spectral properties of $q_{0, t}$ for small $t$. The family $q_{0, t}$ of quadratic forms does not converge as $t$ tends to zero nor do its real-analytic eigenbranches. But a simple renormalization will give convergence.

Fix $\beta>1$ and $\underline{\alpha}$ such that $1<\underline{\alpha}<\beta$. Let $B$ be the function defined in Lemma 4.2. When $\alpha$ tends to 1 , the function $y \mapsto \partial_{y} B(\alpha, y)$ converges to 1 uniformly for $y \in[0, \underline{\alpha}]$. Thus, there exists $\eta$ such that if $1-\eta \leqslant \alpha \leqslant 1$ and $0 \leqslant y \leqslant \underline{\alpha}$ then $\partial_{y} B(\alpha, y) \geqslant \frac{1}{2}$. Choose $t_{0}$ such that $\sqrt{1-t_{0}^{2}}<\eta$ then, for each $t<t_{0}$ and each 
$(x, y) \in \mathcal{T}_{t} \cap\{y \leqslant \underline{\alpha}\} f_{0}(x)<\eta$ so that $\partial_{y} B\left(f_{0}(x), y\right)>0$. We may thus use Lemmas 4.3 and 4.4 . The methods and results of section 4.4 then apply and we define the quadratic form $q_{0, t}$ as previously.

For each $t \in\left[0, t_{0}[\right.$, define the renormalized quadratic form by

$$
q_{t}:=t^{2} \cdot q_{0, t}
$$

with domain $H_{\beta}^{1}(S)$. By Theorem 4.9 , the family $t \mapsto q_{t}$ is real-analytic of type (a) for $t \in] 0,1$ [. In particular, the results of 4.5 apply.

To study the limiting properties of the family $q_{t}$, we re-express $q_{t}$ in a more convenient form: For each $C^{1}$ function $w: S \rightarrow \mathbb{C}$ define

$$
\widetilde{\nabla}_{t} w=\left(\partial_{x} w, t \cdot \partial_{y} w\right) .
$$

Recall that $Y_{\alpha}$ is the inverse of $B_{\alpha}$ and set $f(x)=f_{0}(x)=\sqrt{1-x^{2}}$. Define

$$
\widetilde{\rho}_{t}(a, b)=\frac{Y(f(t a) b)}{b} \cdot\left(\partial_{y} Y(f(a t), b)\right)^{\frac{1}{2}}
$$

and

$$
\left(\partial_{y}(Y(f(a t), b))^{-1} \cdot\left(\begin{array}{cc}
1 & \left(\partial_{\alpha} B \circ Y\right) \cdot f^{\prime}(t a) \\
\left(\partial_{\alpha} B \circ Y\right) \cdot f^{\prime}(t a) & \left(\left(\partial_{\alpha} B \circ Y\right) \cdot f^{\prime}(t a)\right)^{2}+\left(\partial_{y} B\right)^{2}
\end{array}\right)\right.
$$

where the subscript (or first argument) in each $Y$ and $B$ is $f(t \cdot a)$. When comparing $\rho$ and $\tilde{\rho}(Q$ and $\tilde{Q})$ we see that we only miss some powers of $t$ that eventually cancel in the computation leading to Proposition 4.8

This shows that for each $u \in H_{\beta}^{1}(S)$

$$
q_{t}(u)=\int_{S_{-}} \widetilde{\nabla}\left(\widetilde{\rho}_{t} \cdot u\right) \cdot \widetilde{Q}_{t} \cdot \widetilde{\nabla}\left(\widetilde{\rho}_{t} \cdot \bar{u}\right)^{*} d a d b+\int_{S_{+}} \widetilde{\nabla} u \cdot(\widetilde{\nabla} \bar{u})^{*} d a d b
$$

where $S_{-}=[0,1] \times[1, \underline{\alpha}]$ and $S^{+}=[0,1] \times[\underline{\alpha}, \infty]$.

By arguing as in the proof of Theorem 4.9 , one can show that $t \mapsto q_{t}(u)$ is analytic at $t=0.7$

We will now compute the first few terms in the Taylor series in $t$ for $\widetilde{\rho}$ and $\widetilde{Q}$. These functions are analytic on a neighbourhood of $\left[0, t_{0}\left[\times S_{-}\right.\right.$. In particular, in the following, the expressions like $O\left(t^{2}\right)$ are uniform with respect to $(a, b) \in S^{-}$and may be differentiated with respect to $t, a$ and $b$.

We first compute

$$
f(t a)=1-\frac{1}{2} \cdot t^{2} \cdot a^{2}+O\left(t^{4}\right)
$$

and

$$
f^{\prime}(t a)=-t \cdot a+O\left(t^{3}\right) .
$$

Since $\alpha \mapsto Y_{\alpha}$ is analytic and $Y_{1}(b)=b$, it follows from $19 p$ that

$$
Y_{f(t a)}(b)=b+O\left(t^{2}\right) \text {. }
$$

\footnotetext{
${ }^{7}$ However, because $q_{0}$ is not closed on the domain $H_{\beta}^{1}(S)$, the family $q_{t}$ is not analytic at $t=0$ in the sense of Kato.
} 
Moreover, using analyticity, this asymptotic expansion may be differentiated with respect to $(a, b)$. We thus obtain,

$$
Y_{f(t a)}^{\prime}(b)=1+O\left(t^{2}\right)
$$

Substituting these into (16), and differentiating, we find that

$$
\widetilde{\rho}_{t}(a, b)=1+O\left(t^{2}\right), \nabla_{a, b} \widetilde{\rho}_{t}(a, b)=O\left(t^{2}\right) .
$$

Using (19), 20), (21), and (22) we find that

$$
\widetilde{Q}_{t}(a, b)=I+t \cdot a \cdot p(b) \cdot\left(\begin{array}{cc}
0 & 1 \\
1 & 0
\end{array}\right)+O\left(t^{2}\right)
$$

where $I$ is the identity matrix, $O\left(t^{2}\right)$ is a matrix whose operator norm is bounded by a constant times $t^{2}$ as $t$ tends to zero, and $p$ is the polynomial

$$
p(b)=-\left.\partial_{\alpha} B_{\alpha}(b)\right|_{\alpha=1} .
$$

To prove Theorem 1.1 we will need to know that $p(1) \neq 0$.

Lemma 5.1. $p(1)=1$.

Proof. By construction we have $B(\alpha, \alpha)=1$. By differentiating with respect to $\alpha$ and setting $\alpha=1$ we get

$$
\partial_{\alpha} B(1,1)+\partial_{y} B(1,1)=0 .
$$

Since $\partial_{y} B(\alpha, y)=1+O\left((\alpha-1)^{2}\right)$ the claim follows.

\section{Asymptotic separation of VARIABles}

In this section we apply the method of asymptotic separation of variables developed in HlrJdg11 (see also HlrJdg12 ) to the family $q_{t}$. Using the small $t$ asymptotics derived in 95 , we approximate $q_{t}$ to first order with a family of quadratic forms $a_{t}$ for which separation of variables apply. We also derive a non-concentration estimate for eigenfunctions of $q_{t}$.

Notation. In this section and the following sections, we will use $(x, y)$ in place of $(a, b)$ as coordinates for $S=[0,1] \times[1, \infty[$ and unless it is specified otherwise $\|\cdot\|$ is the norm in $L^{2}\left(S, y^{-2} d x d y\right)$.

6.1. Asymptotic approximation. We begin by using the expansions obtained in $\$ 5$ to determine the forms used to approximate $q_{t}$. In particular, by substituting the expansions $(23)$ and $(24)$ into $(18)$ we are led to define

$$
a_{t}(u, v)=\int_{S} \widetilde{\nabla} u \cdot \widetilde{\nabla} \bar{v} d x d y=\int_{S}\left(u_{x} \cdot \bar{v}_{x}+t^{2} \cdot u_{y} \cdot \bar{v}_{y}\right) d x d y
$$

and

$$
b_{t}(u, v)=\int_{S_{-}} \widetilde{\nabla} u \cdot \mathcal{B}(x, y) \cdot \widetilde{\nabla} \bar{v} d x d y
$$

where the operator $\widetilde{\nabla}$ is defined by $\left[15, S_{-}=[0,1] \times[1, \underline{\alpha}]\right.$, and

$$
\mathcal{B}(x, y)=x \cdot p(y) \cdot\left(\begin{array}{ll}
0 & 1 \\
1 & 0
\end{array}\right) .
$$

We wish to show that $q_{t}$ is asymptotic to $a_{t}$ at first order in the sense of HlrJdg11. It will also be used to help derive a key estimate for crossing eigenbranches. However, although $a_{t}$ is a positive quadratic form, the bottom of its 
spectrum tends to 0 so that it is more convenient to use the quadratic form $\widetilde{a_{t}}$ that we now define to control quantities.

Definition 6.1. The quadratic form $\widetilde{a_{t}}$ is defined on $\operatorname{dom}\left(a_{t}\right)$ by

$$
\widetilde{a_{t}}(v)=a_{t}(v)+\|v\|^{2} .
$$

The following proposition can be seen as the beginning of an asymptotic expansion for $q_{t}$.

Proposition 6.2. There exists $C$ such that for each $u, v \in H_{\beta}^{1}(S)$

$$
\left|q_{t}(u, v)-a_{t}(u, v)-t \cdot b_{t}(u, v)\right| \leqslant C \cdot t^{2} \cdot \widetilde{a}_{t}(u)^{\frac{1}{2}} \widetilde{a}_{t}(v)^{\frac{1}{2}}
$$

Proof. We have

$$
\widetilde{\nabla}_{t} \widetilde{\rho}_{t} \cdot u=\widetilde{\rho}_{t} \cdot \widetilde{\nabla}_{t} u+u \cdot \widetilde{\nabla}_{t} \widetilde{\rho}_{t} \cdot
$$

If $y \geqslant \underline{\alpha}$, then $\widetilde{\rho}_{t}$ is identically equal to 1 and $\widetilde{Q}_{t}$ is identically equal to $I$. Hence, by subsituting 29 into (18), we find that $q_{t}(u, v)-a_{t}(u, v)-t \cdot b_{t}(u, v)$ is the sum of the following four terms

$$
\begin{aligned}
& \int_{S_{-}} \widetilde{\nabla}_{t} u \cdot\left(\tilde{\rho}^{2} \cdot \widetilde{Q}_{t}-I-t \cdot \mathcal{B}\right) \cdot \widetilde{\nabla}_{t} v d x d y \\
& \int_{S_{-}} \widetilde{\rho}_{t} \cdot v \cdot\left(\widetilde{\nabla}_{t} \widetilde{\rho}_{t} \cdot \widetilde{Q}_{t} \cdot \widetilde{\nabla}_{t} u\right) d x d y \\
& \int_{S_{-}} \widetilde{\rho}_{t} \cdot u \cdot\left(\widetilde{\nabla}_{t} \widetilde{\rho}_{t} \cdot \widetilde{Q}_{t} \cdot \widetilde{\nabla}_{t} v\right) d x d y \\
& \int_{S_{-}}\left(\widetilde{\nabla}_{t} \widetilde{\rho}_{t} \cdot \widetilde{Q}_{t} \cdot \widetilde{\nabla}_{t} \widetilde{\rho}_{t}\right) \cdot u \cdot v d x d y .
\end{aligned}
$$

In order to estimate these four terms, we use the asymptotic expansions of $\$ 5$. For example, by (24) we have that $(30$ is equal to

$$
\int_{S} \widetilde{\nabla}_{t} u \cdot O\left(t^{2}\right) \cdot \widetilde{\nabla}_{t} v d x d y
$$

Since the operator norm of the matrix $O\left(t^{2}\right)$ is bounded by a constant $C$ times $t^{2}$, we can apply the Cauchy-Schwarz inequality to find that the norm of 30 is bounded by $C \cdot t^{2} \cdot a_{t}(u)^{\frac{1}{2}} \cdot a_{t}(v)^{\frac{1}{2}}$.

Similar arguments show that there is a constant $C$ so that

- 31 is bounded above by $C \cdot t^{2} \cdot a_{t}(u)^{\frac{1}{2}} \cdot\|v\|^{\frac{1}{2}}$

- 32 is bounded above by $C \cdot t^{2}\|u\|^{\frac{1}{2}} \cdot a_{t}(v)^{\frac{1}{2}}$

- 33 is bounded above by $C \cdot t^{2} \cdot\|u\|^{\frac{1}{2}} \cdot\|v\|^{\frac{1}{2}}$.

The claim follows.

6.2. The spectrum of $a_{t}$ via separation of variables. We recall the Fourier decomposition of section 2.2. Since now $w=1$ we thus have, for each $u \in L^{2}\left(S, \frac{d x d y}{y^{2}}\right)$

$$
u^{k}(y)=\int_{0}^{1} u(x, y) \cdot e_{k}(x) d x .
$$

where the latter makes sense for almost every $y$ and defines an element of $L^{2}\left((1, \infty), \frac{d y}{y^{2}}\right)$.

As above, let $\mathcal{D}(\bar{S})$ denote the set of functions $v: S \rightarrow \mathbb{C}$ such that $v$ is the restriction of a compactly supported, smooth function defined in a neighborhood 
of $S$. If $u \in \mathcal{D}$, then each $u^{k}$ is smooth, and a straightforward computation shows that 8

$$
\begin{aligned}
a_{t}(u) & =\sum_{k \in \mathbb{N}} a_{t}\left(u^{k} \otimes e_{k}\right) \\
& =\sum_{k \in \mathbb{N}} \int_{1}^{\infty}\left(t^{2} \cdot \partial_{y} u^{k}(y)^{2}+(k \pi)^{2} \cdot u^{k}(y)^{2}\right) d y
\end{aligned}
$$

We define $\mathcal{D}([1, \infty[)$ to be the set of compactly supported, smooth functions defined on $[1, \infty[$. For $v \in \mathcal{D}([1, \infty))$, each integer $k$, and each $t>0$, we define

$$
a_{t}^{k}(v)=\int_{1}^{\infty}\left(t^{2} \cdot v^{\prime}(y)^{2}+(k \pi)^{2} \cdot v(y)^{2}\right) d y
$$

For $v, w$ in $L^{2}\left(\left[1, \infty\left[, y^{-2} d y\right)\right.\right.$, the inner product is defined by

$$
\langle u, v\rangle_{y}=\int_{1}^{\infty} u(y) \cdot v(y) \frac{d y}{y^{2}} .
$$

Let $L_{\beta}^{2}$ denote the subspace consisting of those functions whose support lies in $[1, \beta]$.

For each $k \in \mathbb{N}$, the quadratic form $a_{t}^{k}$ extends to a closed, densely defined form on the completion of $\mathcal{D}\left(\left[1, \infty[)\right.\right.$ with respect to $v \mapsto a_{t}^{k}(v)^{\frac{1}{2}}+\langle v, v\rangle_{y}^{\frac{1}{2}}$. For $k=0$, we will restrict the domain of $a_{t}^{k}$ to be the completion of those smooth functions whose support lies in $[1, \beta]$.

If $u$ is an eigenfunction of $a_{t}$ with eigenvalue $\lambda$, then for each $v$ in the domain of $a_{t}^{k}$, we have

$$
a_{t}^{k}\left(u^{k}, v\right)=a_{t}\left(u, v \otimes e_{k}\right)=\lambda \cdot\left\langle u, v \otimes e_{k}\right\rangle=\lambda \cdot\left\langle u^{k}, v\right\rangle_{y},
$$

and hence $u^{k}$ is an eigenfunction of $a_{t}^{k}$ with eigenvalue $\lambda$ with respect to $\langle\cdot, \cdot\rangle_{y}$. Thus, each eigenfunction $u$ of $a_{t}$ may be written uniquely as

$$
u=\sum_{k \in \mathbf{N}} u^{k} \otimes e_{k}
$$

where the $k^{\text {th }}$ Fourier coefficient $u^{k}$ is an eigenfunction of $a_{t}^{k}$. Moreover, the spectrum of $a_{t}$ with respect to $\langle\cdot, \cdot\rangle$ is the union of the spectra of $a_{t}^{k}$ with respect to $\langle\cdot, \cdot\rangle_{y}$. In what follows we will often suppress the subscript $y$ from the notation.

The next two lemmas identify the eigenfunctions of $a_{t}^{k}$ for each $k$.

The zero modes are given by the following lemma.

Lemma 6.3. The spectrum of $a_{t}^{0}$ with respect to $\langle\cdot, \cdot\rangle_{y}$ is the set

$$
\left\{t^{2} \cdot\left(\frac{1}{4}+r^{2}\right) \mid r>0 \text { and } 2 r=\tan (r \cdot \ln (\beta))\right\} \text {. }
$$

The eigenspace associated to $t^{2} \cdot\left(\frac{1}{4}+r^{2}\right)$ is spanned by the eigenvector

$$
\psi(y)=y^{\frac{1}{2}} \cdot \cos (r \ln (y))-\frac{y^{\frac{1}{2}}}{2 r} \cdot \sin (r \ln (y)) .
$$

Proof. Suppose that $v$ is an eigenfunction, that is $a_{t}^{0}(v, w)=\lambda \cdot\langle v, w\rangle$ for all $w$. This implies first that

$$
-t^{2} \cdot v^{\prime \prime}(y)=-\frac{\lambda}{y^{2}} \cdot v(y)
$$

\footnotetext{
${ }^{8}$ Here $\otimes$ is the operation defined by $(v \otimes w)(x, y)=v(y) \cdot w(x)$.
} 
holds in the distributional sense. Ellipticity then yields that $v$ is smooth. Moreover, by integrating by parts against a smooth function that is identically equal to 1 near $y=1$, we also find that $v^{\prime}(0)=0$. Let $s$ be such that $s \cdot(1-s)=\lambda / t^{2}$. Then two linearly independent solutions are given by $y^{s}$ and $y^{1-s}$ if $s \neq \frac{1}{2}$ and by $y^{\frac{1}{2}}$ and $y^{\frac{1}{2}} \cdot \ln (y)$ if $s=\frac{1}{2}$. The condition that $\lambda / t^{2}$ is real and nonnegative implies either that $s=\frac{1}{2}+i r$ with $r>0$, that $s \in[0,1 / 2)$ or that $s=\frac{1}{2}$. If $\operatorname{Re}(\mathrm{s})=1 / 2$, then the boundary conditions $v^{\prime}(1)=0$ and $v(\beta)=0$ imply that the solutions take the form given in (37) with $2 r=\tan (r \cdot \ln (\beta))$. If $s \in[0,1 / 2]$, then there are no solutions that satisfy the boundary conditions.

The nonzero modes are given by the following lemma.

Lemma 6.4. For each $t$ and $k$ and eigenvalue $\lambda$ of $a_{t}^{k}$ with respect to $\langle\cdot, \cdot\rangle_{y}$, the associated eigenspace consists of functions of the form $y \mapsto f(\pi k \cdot y / t)$ such that

$$
\begin{aligned}
& \text { i) } f^{\prime \prime}(z)=\left(1-\frac{\lambda}{(t \cdot z)^{2}}\right) \cdot f(z), \\
& \text { ii) } f \in L^{2}\left([1, \infty), \frac{d y}{y^{2}}\right) \\
& \text { iii) } f^{\prime}(\pi \cdot k / t)=0 .
\end{aligned}
$$

Moreover, when $t$ varies, the spectrum is organized into eigenvalues branches $\lambda_{i}(t)$. For each $i$, the function $t \mapsto \lambda_{i}(t)$ is increasing, and

$$
\lim _{t \rightarrow 0} \lambda_{i}(t)=(\pi \cdot k)^{2}
$$

Proof. Integrate by parts as in the proof of Lemma 6.3 , make the change of variables $z \mapsto \pi k \cdot y / 2 t$, and use the boundary conditions. The existence of a complete set of real-analytic branches of eigenvalues and eigenvectors follows from Theorem 3.3 .

For each real-analytic eigenbranch $t \mapsto \lambda_{i}(t)$, the first order variation is obtained by the classical formula

$$
\dot{\lambda}_{i}(t)=\dot{a}_{t}^{k}\left(v_{t}\right)=2 t \int_{1}^{\infty} v_{t}^{\prime}(y)^{2} d y,
$$

where $v_{t}$ is the normalized eigenfunction associated with $\lambda_{i}(t)$. Since the integrand is nonnegative, the eigenvalue branches are increasing.

The fact that each eigenbranch $t \mapsto \lambda_{i}(t)$ limits to $(\pi \cdot k)^{2}$ follows from the methods of HlrJdg11. Alternatively, one can transform the eigenvalue problem into a problem involving a Schrödinger operator with a potential whose minimum value equals $(\pi \cdot k)^{2}$.

Remark 6.5. The eigenfunctions of $a_{t}^{k}$ for positive $k$ may be regarded as Bessel functions since the corresponding differential equation can be transformed into a Bessel equation on the half-line $[i, i \cdot \infty)$.

As a consequence of the identification of the eigenfunctions we have the following Poincaré type inequality.

Lemma 6.6. For each $t \leqslant 2 \pi$, and each $u \in H_{\beta}^{1}(S)$ we have

$$
a_{t}(u) \geqslant \frac{t^{2}}{4} \cdot n(u) .
$$


Proof. We have

$$
a_{t}(u)=\sum_{k} a_{t}^{k}\left(u^{k}\right) \quad \text { and } \quad n(u)=\sum_{K} \int_{1}^{\infty}\left|u^{k}\right|^{2} \frac{d y}{y^{2}} .
$$

Lemmas 6.4 and 6.3 imply

$$
a_{t}^{0}\left(u^{0}\right) \geqslant \frac{t^{2}}{4} \cdot \int_{1}^{\beta}\left|u^{0}\right|^{2} \frac{d y}{y^{2}}
$$

and

$$
a_{t}^{k}\left(u^{k}\right) \geqslant(\pi \cdot k)^{2} \cdot \int_{1}^{\infty}\left|u^{k}\right|^{2} \frac{d y}{y^{2}}
$$

for $k>0$.

In the sequel we will use different kind of projections associated either with the Fourier decomposition $u=\sum u^{k} \otimes e_{k}$ or with the spectral decomposition of $a_{t}$.

More precisely, for each $\ell \in \mathbb{N}$ define the orthogonal projection $\Pi_{\ell}: L_{\beta}^{2}(S) \rightarrow$ $L_{\beta}^{2}(S)$ by

$$
\Pi_{\ell}(v)=v^{\ell}(y) \cdot e_{\ell}(x),
$$

and let $V_{\ell}$ denote the image of $\Pi_{\ell}$. For each $k \in \mathbb{Z}$, we define

$$
\Pi_{\ell<k}(v)=\sum_{\ell<k} v^{\ell}(y) \cdot e_{\ell}(x),
$$

the projection onto $\bigoplus_{0 \leqslant \ell<k} V_{\ell}$.

We also define $P_{a_{t}}^{\lambda}$ to be the orthogonal projection onto the eigenspace of $a_{t}$ associated to the eigenvalue $\lambda$ of $a_{t}$. For a fixed interval $I$, define the $a_{t}$-spectral projection in the energy interval $I$ to be

$$
P_{a_{t}}^{I}(v):=\sum_{\lambda \in \operatorname{spec}\left(a_{t}\right) \cap I} P_{a_{t}}^{\lambda}(v) .
$$

For each eigenvalue $\lambda$ of $a_{t}$, the associated $a_{t}$-eigenspace $W_{\lambda}$ is the orthogonal direct sum $\oplus_{\ell}\left(W_{\lambda}^{\ell} \otimes \operatorname{vect}\left(e_{\ell}\right)\right)$ where $W_{\lambda}^{\ell}$ is the $\lambda$-eigenspace of $a_{t}^{\ell}$ and $\operatorname{vect}\left(e_{\ell}\right)$ is the span of $e_{\ell}$. It follows that

$$
\Pi_{\ell}\left(P_{a_{t}}^{I}(v)\right):=\sum_{\lambda \in \operatorname{spec}\left(a_{t}^{\ell}\right) \cap I} P_{a_{t}}^{\lambda}\left(\Pi_{\ell}(v)\right) .
$$

More generally, for a quadratic form $b$ the notation $P_{b}^{I}$ will always denote the spectral projection onto the interval $I$.

6.3. Asymptotic at first order. In the following, we let $\dot{q}_{t}$ (resp. $\dot{a}_{t}$ ) denote the derivative of $q_{t}$ (resp. $a_{t}$ ) in $t$. The following proposition will allow us to compare $a_{t}$ and $q_{t}$ in the limit $t \rightarrow 0$.

Proposition 6.7 (Asymptotic at first order). There exists a constant $C$ and $t_{0}$ such that, for all $u, v \in H_{\beta}^{1}$ and all $t \leqslant t_{0}$,

$$
\begin{aligned}
\left|q_{t}(u, v)-a_{t}(u, v)\right| & \leqslant C \cdot t \cdot a_{t}(u)^{\frac{1}{2}} \cdot a_{t}(v)^{\frac{1}{2}} \\
\left|\dot{q}_{t}(u)-\dot{a}_{t}(u)\right| & \leqslant C \cdot a_{t}(u) .
\end{aligned}
$$


In HlrJdg11, two real-analytic families of quadratic forms $a_{t}$ and $q_{t}$ satisfying (43) and (44) were said to be asymptotic at first order. We will use the same terminology here.

Proof. One argues as in the proof of Proposition 6.2 paying a little more attention to the terms (31), 32), and (33). For example, to estimate (31), use the CauchySchwarz inequality and 23 to obtain

$$
\left|\widetilde{\nabla}_{t} \widetilde{\rho}_{t} \cdot \widetilde{\nabla}_{t} u\right| \leqslant\left|\widetilde{\nabla}_{t} \widetilde{\rho}_{t}\right| \cdot\left|\widetilde{\nabla}_{t} u\right|=O\left(t^{2}\right) \cdot\left|\widetilde{\nabla}_{t} u\right| .
$$

The Cauchy-Schwarz inequality and Lemma 6.3 give

$$
\int_{S}|v| \cdot\left|\widetilde{\nabla}_{t} u\right| d x d y \leqslant \frac{1}{t \cdot \sqrt{1 / 4+r_{0}^{2}}} \cdot a_{t}(u)^{\frac{1}{2}} \cdot a_{t}(v)^{\frac{1}{2}} .
$$

By combining 45 and 46 and using $(23)$ we find that

$$
\int_{S}\left|\widetilde{\rho}_{t}\right| \cdot|v| \cdot\left|\widetilde{\nabla}_{t} \widetilde{\rho}_{t} \cdot \widetilde{Q}_{t} \cdot \widetilde{\nabla}_{t} u\right| d x d y=O(t) \cdot a_{t}(u)^{\frac{1}{2}} \cdot a_{t}(v)^{\frac{1}{2}} \cdot
$$

Switching the roles of $u$ and $v$, we obtain the same bound for the expression in 32 . Similar methods apply to bound the other terms.

The estimate for $\dot{q}-\dot{a}$ is obtained in a similar way.

\section{Limits OF EIGENVALUE BRANCHES}

Since $q_{t}$ is asymptotic to $a_{t}$ at first order and $a_{t}$ and $\dot{a}_{t}$ are nonnegative quadratic forms, each real-analytic eigenvalue branch $E_{t}$ of $q_{t}$ converges to a finite limit $E_{0}$ as $t$ tends to zero (Theorem 3.4 of HlrJdg11). For the Dirichlet eigenvalue problem on $\mathcal{T}_{t}$, we showed in HlrJdg11 HlrJdg12 that each limit $E_{0}$ has the form $(\pi k)^{2}$ where $k$ is an integer. The methods of HlrJdg11 can be applied to show that the same fact is true in the present context. In this section we highlight the necessary modifications. We also show that if the eigenvalue branch is associated to a cusp form, then $k$ must be positive. This latter fact will be used crucially in the proof of Theorem 1.1

7.1. Non-concentration and first variation. The proof of convergence depends crucially on the following 'non-concentration' result proved for the Dirichlet problem in HlrJdg11.

We will let $\mathcal{D}_{\ell}$ denote the domain of the quadratic form $a_{t}^{\ell}$. We define a quadratic form $\widetilde{a}_{t}^{\ell}: \mathcal{D}_{\ell} \rightarrow \mathbb{C}$ by setting

$$
\tilde{a}_{t}^{\ell}(v)=a_{t}^{\ell}(v)+\|v\|^{2} .
$$

for each $v \in \mathcal{D}_{\ell}$.

Proposition 7.1. (Compare Proposition 9.1 of [HlrJdg11]) Let $\ell \in \mathbb{N}$, let $K$ be a compact subset of $](\pi \ell)^{2}, \infty\left[\right.$, and let $C>0$. There exist positive constants $t_{0}$ and $\kappa$ (that only depend on $\ell, K$ and $C$ ) so that if $E \in K$, if $t<t_{0}$, and if for each $w \in \mathcal{D}_{\ell}$, the function $v \in \mathcal{D}_{\ell}$ satisfies

$$
\left|a_{t}^{\ell}(v, w)-E \cdot\langle v, w\rangle\right| \leqslant C \cdot t \cdot\|w\| \cdot\|v\|
$$

then

$$
\int_{1}^{\infty}\left(\frac{E}{y^{2}}-(\ell \pi)^{2}\right) \cdot|v(y)|^{2} d y \geqslant \kappa \cdot\|v\|^{2}
$$


Proof. If $\ell=0$ and we let $\kappa=\inf (K)>0$, then 47) holds. If $\ell>0$ this follows from Proposition 9.1 of HHlrJdg11] with $\mu=(\pi \ell)^{2}$ and $\sigma(y)=y^{-2}$. See the end of HlrJdg12 for a proof of Proposition 9.1 of HlrJdg11.

In the language of semi-classical analysis, Proposition 7.1 asserts that a quasimode $v$ of order $t$ at energy $E$ does not concentrate at $y=\sqrt{E} /(\ell \pi)$ if $\ell \neq 0$. In $\S 12$ of HlrJdg11, we used non-concentration to derive indirect estimates for $\dot{a}_{t}$. The following Proposition and Corollary make these estimates more transparent and simpler to apply.

Proposition 7.2. Let $\ell \in \mathbb{N}$ and let $K \subset](\pi \ell)^{2}, \infty[$ be compact. For each $\epsilon>0$, there exists $\kappa^{\prime}>0$ and $t_{0}>0$ such that for each $v \in \mathcal{D}_{\ell}$ and $t<t_{0}$

$$
\|v\|^{2} \leqslant \frac{t}{\kappa^{\prime}} \cdot \dot{a}_{t}^{\ell}(v)+\frac{\epsilon}{t^{2}} \cdot N_{\ell}(v, E)^{2} .
$$

where

$$
N_{\ell}(v, E)=\sup _{w \in \mathcal{D}} \frac{\left|a_{t}^{\ell}(v, w)-E\langle v, w\rangle\right|}{\widetilde{a}_{t}^{\ell}(w)^{\frac{1}{2}}}
$$

Proof. From 35 we find that $\dot{a}_{t}^{\ell}(v)=2 t \int_{1}^{\infty} v^{\prime}(y)^{2}$ and hence

$$
t \cdot \dot{a}_{t}^{\ell}(v)=2 \cdot \int_{1}^{\infty}\left(\frac{E}{y^{2}}-(\pi \ell)^{2}\right) \cdot v^{2}+2\left(a_{t}^{\ell}(v)-E \cdot\|v\|^{2}\right) .
$$

If the claim is not true, then for each $\kappa^{\prime}>0$, there exists a sequence $\left(t_{n}\right)_{n \geqslant 1}$ tending to zero and sequences $\left(\tilde{v}_{n}\right)_{n \geqslant 1}, \tilde{v}_{n} \in \mathcal{D},\left(E_{n}\right)_{n \geqslant 1}, E_{n} \in K$ such that

$$
\left\|\tilde{v}_{n}\right\|^{2} \geqslant \frac{t_{n}}{\kappa^{\prime}} \cdot \dot{a}_{\ell}\left(\tilde{v}_{n}\right)+\frac{\epsilon \cdot N_{\ell}\left(\tilde{v}_{n}, E_{n}\right)^{2}}{t_{n}^{2}} .
$$

In particular, since $\dot{a} \geqslant 0$, we have $N\left(\tilde{v}_{n}, E_{n}\right)^{2} \leqslant\left(t_{n}^{2} / \epsilon\right) \cdot\left\|\tilde{v}_{n}\right\|^{2}$. It follows that for each $w \in \mathcal{D}$

$$
\left|\tilde{a}_{t}^{\ell}\left(\tilde{v}_{n}, w\right)-\left(E_{n}+1\right) \cdot\left\langle\tilde{v}_{n}, w\right\rangle\right| \leqslant \frac{t_{n}}{\sqrt{\epsilon}} \cdot\left\|\tilde{v}_{n}\right\| \cdot \tilde{a}_{t}^{\ell}(w)^{\frac{1}{2}} .
$$

Fix $\delta>0$ such that $[-\delta, \delta]+K \subset\left((\ell \pi)^{2}, \infty\right)$. Set $I_{n}=\left[E_{n}-\delta, E_{n}+\delta\right]$ and $v_{n}=P_{a_{t}}^{I_{n}}\left(\tilde{v}_{n}\right)$. Note that $v_{n}=P_{\widetilde{a}_{t}^{e}}^{I_{n}+1}\left(\tilde{v}_{n}\right)$. where $I_{n}+1:=\left[E_{n}-\delta+1, E_{n}+\delta+1\right]$.

We now argue as in the proof of Lemma 2.3 in HlrJdg11: The estimate 51] implies that

$$
\sum_{i \geqslant 0} \frac{\left(\lambda_{i}-E_{n}+1\right)^{2}}{\lambda_{i}}\left|\left\langle\tilde{v}_{n}, \psi_{i}^{\ell}\right\rangle\right|^{2} \leqslant \frac{t_{n}^{2}}{\epsilon}\left\|\tilde{v}_{n}\right\|^{2}
$$

where $\left(\psi_{i}^{\ell}\right)_{i \geqslant 0}$ is a complete orthonormal set of eigenfunctions of $a_{t}^{\ell}$ (with corresponding eigenvalues $\lambda_{i}$ ). By retaining in the sum only the terms for which $\lambda_{i} \notin I_{n}^{\prime}=\left[E_{n}+1-\delta, E_{N}+1+\delta\right]$, we find that

$$
\tilde{a}_{t}^{\ell}\left(\tilde{v}_{n}-v_{n}\right) \leqslant \frac{t_{n}^{2}}{\epsilon} \cdot\left\|\tilde{v}_{n}\right\|^{2} \cdot\left(1+\frac{E_{n}}{\delta}\right) .
$$

Observe that the sequences $\left(v_{n}\right)_{n \geqslant 1},\left(t_{n}\right)_{n \geqslant 1}$ and $\left(E_{n}\right)_{n \geqslant 1}$ depend on the initial choice of $\kappa^{\prime}$ but the preceding estimate gives a constant $C$ that is independent of $\kappa^{\prime}$ such that

$$
\widetilde{a}_{t}^{\ell}\left(\tilde{v}_{n}-v_{n}\right) \leqslant C \cdot t_{n}^{2} \cdot\left\|\tilde{v}_{n}\right\|^{2} .
$$


This implies in particular $\left\|\tilde{v}_{n}-v_{n}\right\|^{2} \leqslant C \cdot t_{n}^{2} \cdot\left\|\tilde{v}_{n}\right\|^{2}$ so that, for $n$ sufficently large, we have $\left\|v_{n}\right\| \leqslant\left\|\tilde{v}_{n}\right\| \leqslant 2\left\|v_{n}\right\|$.

In equation 51 we replace the test function $w$ by $P_{a_{t}^{e}}^{I_{n}}(w)$ and use that the spectral projector is self-adjoint and commutes with $\widetilde{a}_{t}^{\ell}$. We obtain that for each $w \in \mathcal{D}_{\ell}$,

$$
\begin{aligned}
\left|\widetilde{a}_{t}^{\ell}\left(v_{n}, w\right)-\left(E_{n}+1\right) \cdot\left\langle v_{n}, w\right\rangle\right| & \leqslant \frac{t_{n}}{\sqrt{\epsilon}} \cdot\left\|\tilde{v}_{n}\right\| \cdot \tilde{a}_{t}^{\ell}\left(P_{a_{t}^{\ell}}^{I_{n}}(w)\right)^{\frac{1}{2}} \\
& \leqslant C \cdot t_{n} \cdot\left\|v_{n}\right\|\|w\|,
\end{aligned}
$$

where we have used that $\left\|v_{n}\right\|$ is controlling $\left\|\tilde{v}_{n}\right\|$ and that

$$
\tilde{a}_{t}^{\ell}\left(P_{a_{t}^{\ell}}^{I_{n}}(w)\right) \leqslant(\sup (K)+\delta) \cdot\|w\|^{2}
$$

by definition of a spectral projector.

Since $\dot{a}_{t}^{\ell} \leqslant \frac{2}{t} \cdot a_{t}^{\ell}$ and $\dot{a}_{t}^{\ell}$ is a non-negative quadratic form, we also have

$$
\begin{aligned}
\left|\dot{a}_{t}^{\ell}\left(\tilde{v}_{n}\right)^{\frac{1}{2}}-\dot{a}_{t}^{\ell}\left(v_{n}\right)^{\frac{1}{2}}\right| & \leqslant \dot{a}_{t}^{\ell}\left(\tilde{v}_{n}-v_{n}\right)^{\frac{1}{2}} \\
& \leqslant\left(\frac{C}{t} a_{t}^{\ell}\left(\tilde{v}_{n}-v_{n}\right)\right)^{\frac{1}{2}} \\
& \leqslant C \cdot \sqrt{t} \cdot\left\|v_{n}\right\| .
\end{aligned}
$$

Equation 52 implies that we may use Proposition 7.1 to find

$$
\int_{1}^{\infty}\left(\frac{E_{n}}{y^{2}}-(\ell \pi)^{2}\right)\left|v_{n}(y)\right|^{2} d y \geqslant \kappa \cdot\left\|v_{n}\right\|^{2} .
$$

Since 52 also implies $\left|a_{t}^{\ell}\left(v_{n}\right)-E_{n}\left\|v_{n}\right\|^{2}\right| \leqslant C \cdot t_{n} \cdot\left\|v_{n}\right\|^{2}$, using 499 we find that

$$
t_{n} \cdot \dot{a}_{t}^{\ell}\left(v_{n}\right) \geqslant\left(\kappa-C \cdot t_{n}\right) \cdot\left\|v_{n}\right\|^{2} .
$$

On the other hand, the contradiction assumption implies that

$$
\begin{aligned}
\kappa^{\prime} \cdot\left\|v_{n}\right\|^{2} & \geqslant t_{n} \cdot \dot{a}_{t}^{\ell}\left(\tilde{v}_{n}\right) \\
& \geqslant\left(\sqrt{t_{n}} \dot{a}_{t}^{\ell}\left(v_{n}\right)^{\frac{1}{2}}-C t_{n}\left\|v_{n}\right\|^{2}\right)^{2} \\
& \geqslant\left(\left(\kappa-C \cdot t_{n}\right)^{\frac{1}{2}}-C t_{n}\right)^{2} \cdot\left\|v_{n}\right\|^{2} \\
& \geqslant\left(\kappa-C \cdot t_{n}\right)\left\|v_{n}\right\|^{2} .
\end{aligned}
$$

The implied constant $C$ does not depend on $\kappa^{\prime}$ so if we take $\kappa^{\prime}<\kappa$ then choosing $t_{n}$ small enough yields the contradiction.

This proposition yields an estimate for $\dot{a}(w)$ from below in terms of the projection $\Pi_{\ell<k} w$.

Corollary 7.3. Let $k \in \mathbb{Z}^{+}$and let $K \subset \mathbf{R}^{+}$be a compact subset of $](\pi k)^{2}, \infty[$. For each $\varepsilon^{\prime}>0$, there exists $\kappa>0 t_{0}>0$ such that if $E \in K, w \in \operatorname{dom}\left(a_{t}\right)$, and $t<t_{0}$, then

$$
\dot{a}_{t}(w) \geqslant \frac{\kappa}{t} \cdot\left(\left\|\Pi_{\ell<k}(w)\right\|^{2}-\frac{\varepsilon^{\prime}}{t^{2}} \cdot N(w, E)^{2}\right)
$$


where

$$
N(w, E)=\sup _{v \in \operatorname{dom}\left(a_{t}\right)} \frac{\left|a_{t}(w, v)-E \cdot\langle w, v\rangle\right|}{\widetilde{a}_{t}(v)^{\frac{1}{2}}} .
$$

Remark 7.4. The functional $v \mapsto N(v, E)$ is equivalent to the $H^{-1}$-norm of $\left(A_{t}-\right.$ $E)(v)$ where here $A_{t}$ is the operator such that $\left\langle A_{t} u, v\right\rangle=a_{t}(u, v)$ for each $u, v \in$ $\operatorname{dom}\left(a_{t}\right)$.

Proof of 7.3. Since $\dot{a}_{t}$ is block diagonal with respect to the sum $\bigoplus_{\ell} V_{\ell}$ and $\dot{a}_{t}^{\ell} \geqslant 0$, we have

$$
\dot{a}_{t}(w)=\dot{a}_{t}\left(\sum_{\ell=0}^{\infty} w^{\ell} \otimes e_{\ell}\right)=\sum_{\ell} \dot{a}_{t}^{\ell}\left(w^{\ell}\right) \geqslant \sum_{\ell=0}^{k-1} \dot{a}_{t}^{\ell}\left(w^{\ell}\right) .
$$

We may apply Proposition 7.1 with $\varepsilon=\varepsilon^{\prime} / k$ to each term on the right hand side to find that

$$
\dot{a}_{t}(w) \geqslant \frac{\kappa}{t} \cdot\left(\sum_{\ell=0}^{k-1}\left\|w^{\ell}\right\|^{2}-\frac{\varepsilon^{\prime}}{k \cdot t^{2}} \cdot \sum_{\ell=0}^{k-1} N_{\ell}\left(w^{\ell}, E\right)^{2}\right)
$$

where $\kappa$ is the minimum of the $\kappa^{\prime}$ coming from Proposition 47. For each $\ell$, and $v \in \mathcal{D}_{\ell}$, we have

$$
\begin{aligned}
\frac{\left|a_{t}^{\ell}\left(w^{\ell}, v\right)-E \cdot\left\langle w^{\ell}, v\right\rangle\right|}{\widetilde{a}_{t}^{\ell}(v)^{\frac{1}{2}}} & =\frac{\left|a_{t}\left(w^{\ell} \otimes e_{\ell}, v \otimes e_{\ell}\right)-E \cdot\left\langle w^{\ell} \otimes e_{\ell}, v \otimes e_{\ell}\right\rangle\right| .}{\widetilde{a}_{t}\left(v \otimes e_{\ell}\right)^{\frac{1}{2}}} \\
& =\frac{\left|a_{t}\left(w, v \otimes e_{\ell}\right)-E \cdot\left\langle w, v \otimes e_{\ell}\right\rangle\right| .}{\widetilde{a}_{t}\left(v \otimes e_{\ell}\right)^{\frac{1}{2}}}
\end{aligned}
$$

and hence $N_{\ell}\left(w^{\ell}, E\right) \leqslant N(w, E)$. We also have $\sum_{\ell<k}\left\|w^{\ell}\right\|^{2}=\left\|\Pi_{\ell<k}(w)\right\|^{2}$, and the claim follows.

7.2. The spectral projection $w_{t}$. The bounds proved in $\$ 7.1$ depend on a bound on $N(w, E)$. In this subsection, we show that if $w$ is an $a_{t}$-spectral projection of a $q_{t}$-eigenfunction in an interval containing the eigenvalue $E$, then $N(w, E)$ is of order $t$.

We start with a real-analytic eigenfunction branch $u_{t}$ for $q_{t}$ with associated realanalytic eigenvalue branch $E_{t}$. We let

$$
w_{t}^{I}:=P_{a_{t}}^{I}\left(u_{t}\right)
$$

where we recall that $P_{a_{t}}^{I}$ denotes the spectral projector on the interval $I$ that is associated to $a_{t}$. In the sequel, in arguments for which the interval $I$ is fixed, this notation will be often abbreviated to $w_{t}$.

Let $E_{0}$ denote the limit of $E_{t}$ as $t$ tends to zero. The following two lemmas express the fact that the projection $w_{t}^{I}$ is an order $t$ quasimode for $a_{t}$ at energy $E_{t}$.

The following lemma is similar to Lemma 2.3 in HlrJdg11.

Lemma 7.5. If I is a compact interval whose interior contains $E_{0}$, then there exist $t_{0}>0$ and $C$ such that if $t<t_{0}$, then

$$
a_{t}\left(u_{t}-w_{t}^{I}\right)+\left\|u_{t}-w_{t}^{I}\right\|^{2} \leqslant C \cdot t^{2} \cdot\left\|u_{t}\right\|^{2}
$$

Proof. Using the fact that $u_{t}$ is an eigenfunction of $q_{t}$ and that $a_{t}$ and $q_{t}$ are asymptotic at first order, for each $w \in H_{\beta}^{1}$

$$
\left|a_{t}\left(u_{t}, w\right)-E_{t}\left\langle u_{t}, w\right\rangle\right| \leqslant C \cdot t \cdot \widetilde{a}_{t}\left(u_{t}\right)^{\frac{1}{2}} \widetilde{a}_{t}(w)^{\frac{1}{2}} .
$$


Observe that letting $w=u_{t}$ yields that $a_{t}\left(u_{t}\right) \leqslant \frac{E_{t}}{1-C t^{2}} \cdot\left\|u_{t}\right\|^{2}$. Moreover the former equation can be rewritten as

$$
\left|\widetilde{a}_{t}\left(u_{t}, w\right)-\tilde{E}_{t}\left\langle u_{t}, w\right\rangle\right| \leqslant C \cdot t \cdot \widetilde{a}_{t}\left(u_{t}\right)^{\frac{1}{2}} \cdot \widetilde{a}_{t}(w)^{\frac{1}{2}},
$$

where $\tilde{E}_{t}:=E_{t}+1$. We may now follow the proof of Lemma 2.3 in HlrJdg11 observing that $P_{a_{t}}^{I}=P_{\widetilde{a}_{t}}^{I+\{1\}}$. This yields a constant $C$ such that

$$
\begin{aligned}
a_{t}\left(u_{t}-w_{t}^{I}\right)+\left\|u_{t}-w_{t}^{I}\right\|^{2} & \leqslant C \cdot t^{2} \cdot \widetilde{a}_{t}\left(u_{t}\right) \\
& \leqslant \frac{C \cdot t^{2}}{1-C \cdot t^{2}} \cdot\left\|u_{t}\right\|^{2}, \\
& \leqslant C^{\prime} \cdot t^{2} \cdot\left\|u_{t}\right\|^{2} .
\end{aligned}
$$

The claim follows.

Remark 7.6. Lemma 7.5 implies that most of the mass of $u_{t}$ lies in its projection, $w_{t}^{I}$, onto the energy interval $I$. More precisely, for $t$ small we have

$$
(1-C \cdot t) \cdot\left\|u_{t}\right\| \leqslant\left\|w_{t}^{I}\right\| \leqslant\left\|u_{t}\right\| .
$$

where $C$ is the constant in Lemma 7.5

Lemma 7.7. If I is a compact interval whose interior contains $E_{0}$, then there exist $t_{0}>0$ and $C$ such that if $t<t_{0}$, then

$$
N\left(w_{t}^{I}, E_{t}\right) \leqslant C \cdot t \cdot\left\|w_{t}^{I}\right\| .
$$

Proof. For each $w \in H_{\beta}^{1}$, we have

$$
a_{t}\left(w_{t}, w\right)=a_{t}\left(u_{t}, w\right)-a_{t}\left(u_{t}-w_{t}, w\right)
$$

so that the Cauchy-Schwarz inequality and the preceding lemma imply

$$
\left|a_{t}\left(w_{t}, w\right)-a_{t}\left(u_{t}, w\right)\right| \leqslant C \cdot t\left\|u_{t}\right\| a_{t}(w)^{\frac{1}{2}} .
$$

We also have using Cauchy-Schwarz and the preceding lemma

$$
\left|\left\langle u_{t}, w\right\rangle-\left\langle w_{t}^{I}, w\right\rangle\right| \leqslant C \cdot t \cdot\left\|u_{t}\right\|\|w\| .
$$

We now start again from (57). First, in the bounding term, we have already seen that we could replace $\tilde{a}_{t}\left(u_{t}\right)^{\frac{1}{2}}$ by $C\left\|u_{t}\right\|$. Thus from the triangle inequality, (57) and the two preceding estimates we obtain

$$
\begin{aligned}
\left|a_{t}\left(w_{t}^{I}, w\right)-E\left\langle w_{t}^{I}, w\right\rangle\right| & \leqslant C \cdot t\left\|u_{t}\right\| \cdot\left(a_{t}(w)^{\frac{1}{2}}+\|w\|\right) \\
& \leqslant C \cdot t \cdot\left\|u_{t}\right\| \cdot \widetilde{a}_{t}(w)^{\frac{1}{2}} .
\end{aligned}
$$

The claim follows using (58).

Lemma 7.7 has the following corollary that expresses, in the language of semiclassical analysis, that $w_{t}^{I}$ is an order $t$ quasimode.

Corollary 7.8. If $I$ is a compact interval that contains $E_{0}$ there exists $C$ and $t_{0}>0$ such that, for $t<t_{0}$ and each $v \in \operatorname{dom}\left(a_{t}\right)$, we have

$$
\left|a_{t}\left(w_{t}^{I}, v\right)-E_{t}\left\langle w_{t}^{I}, v\right\rangle\right| \leqslant C \cdot t \cdot\left\|w_{t}^{I}\right\| \cdot\|v\| .
$$


Proof. Since $P_{a_{t}}^{I}$ is a spectral projector, we have

$$
\left|a_{t}\left(w_{t}^{I}, v\right)-E_{t}\left\langle w_{t}^{I}, v\right\rangle\right|=\left|a_{t}\left(w_{t}^{I}, P_{a_{t}}^{I} v\right)-E_{t}\left\langle w_{t}^{I}, P_{a_{t}}^{I} v\right\rangle\right|,
$$

and hence Lemma 7.7 implies

$$
\left|a_{t}\left(w_{t}^{I}, v\right)-E_{t}\left\langle w_{t}^{I}, v\right\rangle\right| \leqslant C \cdot\left\|w_{t}^{I}\right\| \cdot \widetilde{a}_{t}\left(P_{a_{t}}^{I}(v)\right)^{\frac{1}{2}} .
$$

Since $\widetilde{a}_{t}\left(P_{a_{t}}^{I}(v)\right)^{\frac{1}{2}} \leqslant(1+\sup (I))^{\frac{1}{2}} \cdot\|v\|$, the claim follows.

7.3. Limits of eigenvalue branches. By combining Lemma 7.7 with Corollary 7.3 we prove the following.

Theorem 7.9 (Compare Theorem 13.1 HlrJdg11]). Let $\left(E_{t}, u_{t}\right)$ be an eigenbranch of $q_{t}$ then there exists $k \in \mathbb{N}$ such that

$$
\lim _{t \rightarrow 0} E_{t}=(k \cdot \pi)^{2} .
$$

Proof. Suppose to the contrary that $E_{0}$ is not of the form $(k \cdot \pi)^{2}$ where $k$ is an integer. Let $n=\inf \left\{\ell \in \mathbb{N} \mid(\pi \ell)^{2}>E_{0}\right\}$. Choose a compact interval $I \subset$ ] $(n-1)^{2} \pi^{2}, n^{2} \pi^{2}$ [ whose interior contains $E_{0}$.

Let $u_{t}$ be a real-analytic eigenfunction branch of $q_{t}$ associated to $E_{t}$. As before, let $w_{t}^{I}=P_{a_{t}}^{I}\left(u_{t}\right)$ be the projection of $u_{t}$ onto the modes of $a_{t}$ that have energy lying in $I$. Since $I$ is fixed in the rest of this argument, we abbreviate the notation and simply write $w_{t}:=w_{t}^{I}$.

If $\ell \geqslant n$, according to Lemma 6.4 the eigenvalues branches of $a_{t}^{n}$ are increasing and limit to $n^{2} \pi^{2}$. It follows that for each $t$, each eigenvalue of $a_{t}^{\ell}$ is at least $(\pi n)^{2}$. Thus, since $\sup (I)<(\pi n)^{2}$, equation 42 implies that

$$
\Pi_{\ell<n}\left(w_{t}\right)=w_{t} .
$$

Let $C$ be as in Lemma 7.7 , and apply Lemma 7.3 with $\varepsilon^{\prime}=1 /\left(2 C^{2}\right)$ to obtain $\kappa$ so that

$$
\dot{a}_{t}\left(w_{t}\right) \geqslant \frac{\kappa}{2 t} \cdot\left\|w_{t}\right\|^{2}
$$

It follows that $\dot{a}_{t}\left(w_{t}\right) /\left\|w_{t}\right\|^{2}$ is not integrable. This contradicts Theorem 4.2 of HlrJdg11 which we state below as Theorem 7.10

Theorem 7.10 (HlrJdg11 Theorem 4.2). Let $q_{t}$ be asymptotic to $a_{t}$ at first order, and suppose that for each $t>0$, we have

$$
0 \leqslant \dot{a}_{t}(v) \leqslant t^{-1} \cdot a_{t}(v) .
$$

Let $t \mapsto E_{t}$ be a real analytic eigenbranch of $q_{t}$ that converges to a limit $E_{0}$ as $t$ tends to 0 and let $V_{t}$ be the associated eigenspace.

If $t \mapsto u_{t} \in V_{t}$ is continuous on the complement of a countable set, then the function

$$
t \mapsto \frac{\dot{a}_{t}\left(P_{a_{t}}^{I}\left(u_{t}\right)\right)}{\left\|P_{a_{t}}^{I}\left(u_{t}\right)\right\|^{2}}
$$

is integrable on each interval of the form $\left(0, t^{*}\right]$.

The next proposition will be the starting point of the contradiction argument in the following sections. It says that a cusp form eigenbranch cannot limit to 0 . Heuristically, the zeroth Fourier coefficient of a cusp form vanishes identically whereas an eigenvalue branch that limits to 0 must eventually have nontrivial zeroth 
Fourier mode. However, because we have made a nontrivial change of variables, this fact requires an argument.

Proposition 7.11. If $E_{t}$ is a real-analytic cusp form eigenvalue branch of $q_{t}$, then the integer $k$ appearing in (59) is positive.

Proof. Suppose to the contrary that $\lim _{t \rightarrow 0} E_{t}=0$. Set $I=[0,1]$ and consider $w_{t}=w_{t}^{I}$ defined as in 56 . If $\ell>0$, the restriction of $a$ to $V_{\ell}$ is bounded below by $\pi^{2}>1$, thus we have $\Pi_{0}\left(w_{t}\right)=w_{t}$. On the other hand, the projection of $u_{t}$ onto $\bigoplus_{\ell>0} V_{\ell}$ equals $u_{t}-u_{t}^{0} \otimes 1$. Let $v_{t}^{0}$ denote the projection of $u_{t}-w_{t}$ onto $V_{0}$.

Since each $V_{\ell}$ is a direct sum of eigenspaces of $a$, we have

$$
a_{t}\left(u_{t}-w_{t}\right)=a_{t}\left(v_{t}^{0}\right)+a_{t}\left(u-u_{t}^{0} \otimes 1\right) .
$$

The quadratic form $a$ is nonnegative and the restriction of $a_{t}$ to $\bigoplus_{\ell>0} V_{\ell}$ is bounded below by $\pi^{2}$. Hence $a\left(u_{t}-w_{t}\right) \geqslant \pi^{2} \cdot\left\|u_{t}-u_{t}^{0} \otimes 1\right\|^{2}$. By Lemma 7.5 we have

$$
a_{t}\left(u_{t}-w_{t}\right) \leqslant C \cdot t^{2} \cdot\left\|u_{t}\right\|^{2} .
$$

Therefore, $\left\|u_{t}-u_{t}^{0} \otimes 1\right\|^{2} \leqslant C^{\prime} \cdot t^{2} \cdot\left\|u_{t}\right\|^{2}$, and hence

$$
\left\|u_{t}^{0}\right\|_{L^{2}([1, \beta])}^{2} \geqslant\left(1-C^{\prime} t^{2}\right) \cdot\left\|u_{t}\right\|^{2}
$$

for small $t$.

To obtain a contradiction, we will bound $\left\|u_{t}^{0}\right\|$ from above. Towards this end, we will compare $u_{t}^{0}$ with $\Phi_{0, t}\left(u_{t}\right)$ (see $\S 4.3$ ). In particular, Lemma 4.7 implies that $\Phi_{0, t}\left(u_{t}\right)$ is a cusp form for $\mathcal{E}$, and hence for each $y \in[1, \beta]$

$$
\int_{0}^{1} \eta(t \cdot x, y) \cdot u_{t}(x, b(t \cdot x, y)) d x=0
$$

where $b(x, y)=B\left(\sqrt{1-x^{2}}, y\right)$ and $\eta(x, y)=(y / b(x, y)) \cdot \sqrt{\partial_{y} B\left(\sqrt{1-x^{2}}, y\right)}$. (See $\S 4.2$ for the defintion of $B$.) Thus, for all $y \in[1, \beta]$ we have

$$
\begin{aligned}
u_{t}^{0}(y)= & \int_{0}^{1} u_{t}(x, y) d x \\
= & \int_{0}^{1} u_{t}(x, y)-\eta(t \cdot x, y) u_{t}(x, b(t \cdot x, y)) d x \\
= & \int_{0}^{1}(1-\eta(t \cdot x, y)) u_{t}(x, y) d x \\
& +\int_{0}^{1} \eta(t \cdot x, y)\left[u_{t}(x, y)-u_{t}(x, b(t \cdot x, y))\right] d x
\end{aligned}
$$

Let $r_{1}$ and $r_{2}$ denote, respectively, the two integrals on the right-hand side this equation. Thus, $u_{t}^{0}=r_{1}+r_{2}$ where we regard both $r_{1}$ and $r_{2}$ as functions of $y \in[1, \beta]$.

To bound $\left\|r_{1}\right\|$ and $\left\|r_{2}\right\|$, we will repeatedly use the following well-known fact: For each $f \in L^{2}([0,1])$, we have

$$
\left|\int_{0}^{1} f(x) d x\right|^{2} \leqslant \int_{0}^{1}|f(x)|^{2} d x .
$$

Using the properties of $B$, one finds that there exists $C$ such that

$$
\sup \{|\eta(t \cdot x, y)-1|,(x, y) \in[0,1] \times[1, \beta]\} \leqslant C \cdot t
$$


for small $t$. It follows that there exists a (perhaps different) constant $C$ so that

$$
\left\|r_{1}\right\|_{L^{2}([1, \beta])}^{2} \leqslant \int_{1}^{\beta} \int_{0}^{1}\left|(1-\eta(t \cdot x, y)) u_{t}(x, y)\right|^{2} d x d y \leqslant C \cdot t^{2} \cdot\left\|u_{t}\right\|^{2}
$$

Using the fundamental theorem of calculus and the Cauchy-Schwarz inequality we find that

$$
\left|u_{t}(x, b(t \cdot x, y))-u_{t}(x, y)\right|^{2} \leqslant|b(t \cdot x, y)-y| \cdot \int_{y}^{b(t \cdot x, y)}\left|\partial_{2} u_{t}(x, z)\right|^{2} d z
$$

where here $\partial_{2}$ denotes the derivative with respect to the second variable. Using the properties of $B$, we find that there exists $C^{\prime}$ so that

$$
\sup \{|b(t \cdot x, y)-y|,(x, y) \in[0,1] \times[1, \beta]\} \leqslant C^{\prime} \cdot t
$$

for small $t$. It follows that the interval $[y, b(t \cdot x, y)]$ is always a subset of the interval $\left[y-C^{\prime} \cdot t, y+C^{\prime} \cdot t\right]$, so that we obtain the bound

$$
\left|u_{t}(x, b(t \cdot x, y))-u_{t}(x, y)\right|^{2} \leqslant C^{\prime} \cdot t \cdot \int_{|y-z| \leqslant C^{\prime} \cdot t}\left|\partial_{2} u_{t}(x, z)\right|^{2} d z .
$$

Thus we may estimate $\left\|r_{2}\right\|$ as follows:

$$
\begin{aligned}
\left\|r_{2}\right\|_{L^{2}([1, \beta])}^{2} & \leqslant \int_{1}^{\beta} \int_{0}^{1}\left|\eta(t \cdot x, y)\left[u_{t}(x, y)-u_{t}(x, b(t \cdot x, y))\right]\right|^{2} d x d y \\
& \leqslant C \cdot t \int_{1}^{\beta} \int_{0}^{1} \int_{|y-z| \leqslant C^{\prime} \cdot t}\left|\partial_{2} u_{t}(x, z)\right|^{2} d z d x d y \\
& \leqslant C \cdot t^{2} \cdot \int_{1}^{\beta} \int_{0}^{1}\left|\partial_{2} u_{t}(x, z)\right|^{2} d z d x
\end{aligned}
$$

Here the constant $C$ may vary from line to line. Since $a_{t}$ and $q_{t}$ are asymptotic at first order

$$
t^{2} \int_{S}\left|\partial_{2} u_{t}(x, z)\right|^{2} d x d z \leqslant a_{t}\left(u_{t}\right) \leqslant\left(E_{t}+C \cdot t\right) \cdot\left\|u_{t}\right\|^{2}
$$

for sufficiently small $t$. This yields

$$
\left\|r_{2}\right\|_{L^{2}([1, \beta])} \leqslant C\left(E_{t}+C \cdot t\right)^{\frac{1}{2}} \cdot\left\|u_{t}\right\|
$$

By combining estimates 64 and 65 , we find that

$$
\left\|u_{t}^{0}\right\|_{L^{2}([1, \beta])} \leqslant C \cdot\left(E_{t}+t\right)^{\frac{1}{2}} \cdot\left\|u_{t}\right\| .
$$

for $t$ small and some constant $C$. If $E_{t}$ were to tend to zero as $t$ tends to zero, then we would have a contradiction to 62 for small $t$.

7.4. Bounds on the first variation of the eigenvalue. We can also use the nonconcentration of the spectral projection to give an $O\left(t^{-1}\right)$ lower bound on the first variation, $\dot{E}_{t}$, when the projection of the eigenfunction onto the 'small' modes is significant:

Proposition 7.12. Let $I$ be a compact interval whose interior contains $E_{0}$ and $I \subset\left((k-1)^{2} \pi^{2},(k+1)^{2} \pi^{2}\right)$ and let $w_{t}=P_{a_{t}}^{I}\left(u_{t}\right)$ (see $\left.(56)\right)$. For each $\delta>0$, there exists $\kappa^{\prime}>0$ and $t_{0}>0$ so that if $t<t_{0}$ and

$$
\left\|\Pi_{\ell<k}\left(w_{t}\right)\right\| \geqslant \delta \cdot\left\|u_{t}\right\|
$$


then

$$
\dot{E}_{t} \geqslant \frac{\kappa^{\prime}}{t}
$$

Proof. Using the Cauchy-Schwarz inequality and the nonnegativity of $\dot{a}_{t}$ we have

$$
\dot{a}_{t}\left(u_{t}\right) \geqslant \dot{a}_{t}\left(w_{t}\right)-\dot{a}_{t}\left(w_{t}\right)^{\frac{1}{2}} \cdot \dot{a}_{t}\left(u_{t}-w_{t}\right)^{\frac{1}{2}} .
$$

It follows from 26 that for all $v \in \operatorname{Dom}\left(a_{t}\right)$

$$
\dot{a}_{t}(v) \leqslant 2 t^{-1} \cdot a_{t}(v) .
$$

and hence $\dot{a}_{t}\left(w_{t}\right)^{\frac{1}{2}} \leqslant \sqrt{2} \cdot t^{-\frac{1}{2}} \cdot a\left(w_{t}\right)^{\frac{1}{2}} \leqslant t^{-\frac{1}{2}} \cdot(2 \sup (I))^{\frac{1}{2}} \cdot\left\|w_{t}\right\|$. Moreover, by combining this with Lemma 7.5 we find $\dot{a}_{t}\left(u_{t}-w_{t}\right) \leqslant C t\left\|u_{t}\right\|^{2}$.

Thus, from 68 we obtain

$$
\dot{a}_{t}\left(u_{t}\right) \geqslant \dot{a}_{t}\left(w_{t}\right)-C \cdot\left\|u_{t}\right\|^{2} .
$$

Hence by applying Lemma 7.5 we have

$$
\begin{aligned}
\dot{E}_{t} \cdot\left\|u_{t}\right\|^{2} & =\dot{q}\left(u_{t}\right) \\
& \geqslant \dot{a}_{t}\left(u_{t}\right)-C \cdot a\left(u_{t}\right) \\
& \geqslant \dot{a}_{t}\left(w_{t}\right)-C \cdot\left\|u_{t}\right\|^{2}-C \cdot q\left(u_{t}\right) \\
& \geqslant \dot{a}_{t}\left(w_{t}\right)-C \cdot\left\|u_{t}\right\|^{2}
\end{aligned}
$$

for $t$ sufficiently small.

As in the proof of Theorem 7.9, we have $\Pi_{\ell<k+1}\left(w_{t}\right)=w_{t}=\Pi_{\ell<k}\left(w_{t}\right)+$ $\Pi_{k}\left(w_{t}\right)$. Since $\dot{a}_{t}$ is non-negative and 'block-diagonal' we have

$$
\dot{a}_{t}\left(w_{t}\right) \geqslant \dot{a}_{t}\left(\Pi_{\ell<k}\left(w_{t}\right)\right) \text {. }
$$

Let $C$ be as in Lemma 7.7, and apply Lemma 7.3 with $\varepsilon^{\prime}=\delta^{2} /\left(2 C^{2}\right)$ to obtain $\kappa$ so that

$$
\begin{aligned}
\dot{a}_{t}\left(w_{t}\right) & \geqslant \frac{\kappa}{t}\left(\left\|\Pi_{\ell<k}\left(w_{t}\right)\right\|^{2}-\frac{\delta^{2}}{2}\left\|w_{t}\right\|^{2}\right) \\
& \geqslant \frac{\kappa \cdot \delta^{2}}{2 t} \cdot\left\|w_{t}\right\|^{2}
\end{aligned}
$$

for $t$ sufficiently small. Estimate 58 implies that $\left\|w_{t}\right\|^{2} \geqslant \frac{1}{2}\left\|u_{t}\right\|^{2}$ for $t$ small, and therefore by combining the above inequalities, we prove the claim.

In contrast to Proposition 7.12, we have the following.

Lemma 7.13. There exists $t_{0}>0$ and $C$ such that if $t<t_{0}$, then

$$
\frac{\dot{E}_{t}}{E_{t}} \leqslant \frac{2}{t}+3 C \text {. }
$$

Proof. It follows from (26) that for all $v \in \operatorname{Dom}\left(a_{t}\right)$

$$
\dot{a}_{t}(v) \leqslant 2 t^{-1} \cdot a_{t}(v) .
$$

From (43), there exists $C$ so that for sufficiently small $t$

$$
a_{t}(v) \leqslant(1+C \cdot t) \cdot q_{t}(v),
$$

and

$$
\dot{q}_{t}(v) \leqslant \dot{a}_{t}(v)+C \cdot a_{t}(v)
$$


Thus, if $u_{t}$ is the real-analytic eigenfunction branch of $q_{t}$ associated to $E_{t}$, then

$$
\begin{aligned}
\dot{E}_{t} \cdot\left\|u_{t}\right\|^{2} & =\dot{q}_{t}\left(u_{t}\right) \\
& \leqslant \dot{a}_{t}\left(u_{t}\right)+C \cdot a_{t}\left(u_{t}\right) \\
& \leqslant\left(2 \cdot t^{-1}+C\right) \cdot a_{t}\left(u_{t}\right) \\
& \leqslant\left(2 \cdot t^{-1}+C\right) \cdot(1+C \cdot t) \cdot q_{t}\left(u_{t}\right) \\
& =\left(2 \cdot t^{-1}+C\right) \cdot(1+C \cdot t) \cdot E_{t} \cdot\left\|u_{t}\right\|^{2} .
\end{aligned}
$$

By choosing $t_{0}$ sufficiently small, we obtain the claim.

\section{PRoOF OF THE MAIN THEOREM}

Proposition 3.8 reduces the proof of Theorem 1.1 to the following.

Theorem 8.1. The family $t \mapsto q_{t}$ does not have a real-analytic cusp form eigenbranch.

The proof of Theorem 8.1 will be by contradiction. We will assume that there exists a real-analytic cusp form eigenvalue branch, $E_{t}$. By the results of \&77 we have

$$
\lim _{t \rightarrow 0} E_{t}=(\pi \cdot k)^{2} .
$$

where $k$ is positive. We aim to contradict the positivity of $k$.

8.1. Choosing $\beta$. We recall that our construction of the quadratic form $q_{t}$ depends on a parameter $\beta$, by forcing the zeroth Fourier coefficient of the elements of $\operatorname{dom}\left(q_{t}\right)$ to vanish for $y>\beta$.

The proof of Theorem 8.1 will rely on the estimates of solutions to ordinary differential equations made in Appendix B. To make the estimates less tedious, we will choose $\beta$ to be sufficiently close to 1 , where 'sufficiently close' will be determined by the integer $k$ that appears in $(72)$.

However, the construction of the quadratic form $q_{t}$ depends on $\beta 9^{9}$ Therefore, the integer $k$ that appears in 72 depends a priori on $\beta$. In order to avoid circularity of reasoning, we will prove the following.

Proposition 8.2. Let $E_{t}$ be a real-analytic eigenvalue branch associated to a realanalytic cusp form eigenfunction branch of $t \mapsto q_{t}^{\beta}$. For each $\beta^{\prime}>1$, the family $t \mapsto$ $q_{t}^{\beta^{\prime}}$ has a real-analytic cusp form eigenfunction branch with associated eigenvalue branch $E_{t} 10$

Proof. For each fixed $t$, since $E_{t}$ corresponds to a cusp form, it belongs to the spectrum of $q_{t}^{\beta^{\prime}}$ for all $\beta^{\prime}$ (see Lemma 2.7. By Theorem 3.3, there exists a realanalytic eigenvalue branch $s \mapsto \bar{E}_{s}^{t}$ of $s \mapsto q_{s}^{\beta^{\prime}}$ such that $\bar{E}_{t}^{t}=E_{t}$. Since $s \mapsto q_{s}^{\beta^{\prime}}$ has only countably many real-analytic eigenvalue branches, there exists some branch $s \mapsto \bar{E}_{s}^{t}$ such that the set of $t^{\prime}$ with $\bar{E}_{t^{\prime}}^{t}=E_{t^{\prime}}$ has an accumulation point. Thus, by real-analyticity, we have $\bar{E}_{t^{\prime}}^{t}=E_{t^{\prime}}$ for all $t^{\prime}$.

If for each $t$, the dimension of the eigenspace $V_{t}$ of $q_{t}^{\beta^{\prime}}$ associated to $E_{t}$ is greater than one, then one can argue as in the proof of Proposition 3.7 to obtain a realanalytic cusp form eigenfunction branch of $q_{t}^{\beta^{\prime}}$ associated to $E_{t}$.

\footnotetext{
${ }^{9}$ We have suppressed this dependence from notation until now.

${ }^{10}$ The respective eigenfunction branches will not be the same if $\beta \neq \beta^{\prime}$.
} 
Otherwise, by real-analyticity, for each $t$ in the complement of a discrete set $A$ of $t$ we have $\operatorname{dim}\left(V_{t}\right)=1$. Let $t \mapsto u_{t}^{\beta^{\prime}}$ be a real-analytic eigenfunction branch of $q_{t}^{\beta^{\prime}}$ associated to $E_{t}$.

Let $t \mapsto u_{t}^{\beta}$ denote a real-analytic eigenfunction branch of $q_{t}^{\beta}$ associated to $E_{t}$. For each $t$, the pull-back of $u_{t}$ by the diffeomorphism $\varphi_{0, t}^{\beta}$ is a cusp form of $\mathcal{E}$ on $\mathcal{T}_{t}$. In turn, for each $t$, the pull-back of $u_{t} \circ \varphi_{0, t}^{\beta}$ by $\left(\varphi_{0, t}^{\beta^{\prime}}\right)^{-1}$ is a cusp form eigenfunction of $q_{t}^{\beta^{\prime}}$. Hence, the eigenfunction $u_{t}^{\beta^{\prime}}$ is a cusp form for $t \notin A$. Thus, by Corollary 3.6. the branch $t \mapsto u_{t}^{\beta^{\prime}}$ is a real-analytic cusp form eigenbranch of $q_{t}^{\beta^{\prime}}$.

As a consequence of Proposition 8.2 we may fix $\beta$ to satisfy ${ }^{11}$

$$
1<\beta<\frac{k}{k-1} .
$$

It follows that for each $\ell<k$ and $y \in[1, \beta]$ we have

$$
(\pi \cdot \ell)^{2}-\frac{E_{t}}{y^{2}}<0
$$

as soon as $t$ is small enough.

In what follows, we will drop $\beta$ from the notation for $q_{t}^{\beta}$.

8.2. Tracking. In this section we show that there exists a real-analytic eigenvalue branch of $a_{t}^{k}$, that we denote by $\lambda_{t}^{*}$ such that $\left|\lambda_{t}^{*}-E_{t}\right|$ is at most of order $t$. In $\$ 8.4$ we will show to the contrary that $\left|\lambda_{t}^{*}-E_{t}\right|$ is at least of order $t^{\frac{2}{3}}$. This will provide the desired contradiction.

Theorem 8.3 (Tracking). If $E_{t}$ is a cusp form eigenvalue branch of $q_{t}$ with positive limit $(k \pi)^{2}$, then there exists $t_{0}>0, C>0$, and a real-analytic eigenvalue branch $\lambda_{t}^{*}$ of $a_{t}^{k}$ so that for each $t<t_{0}$,

$$
\operatorname{spec}\left(a_{t}^{k}\right) \bigcap[E-C t, E+C t]=\left\{\lambda_{t}^{*}\right\} .
$$

Proof. Let $u_{t}$ denote a real-analytic eigenfunction branch of $q_{t}$ associated to the eigenvalue branch $E_{t}$. Let $I \subset\left((k-1)^{2} \pi^{2},(k+1)^{2} \pi^{2}\right)$ be a compact interval that contains $k^{2} \pi^{2}$ in its interior. Let $w_{t}=P_{a_{t}}^{I}\left(u_{t}\right)$ the associated spectral projection.

By Corollary 7.8 and the fact that $\Pi_{k}$ is an orthogonal projection that commutes with $a_{t}$, there exist positive constants $C_{q m}$ and $t_{1}$ so that if $t<t_{1}$ and $v \in \mathcal{D}$, then

$$
\left|a_{t}\left(\Pi_{k}\left(w_{t}\right), \Pi_{k}(v)\right)-E_{t} \cdot\left\langle\Pi_{k}\left(w_{t}\right), \Pi_{k}(v)\right\rangle\right| \leqslant \frac{C_{q m}}{2} \cdot t \cdot\left\|w_{t}\right\| \cdot\left\|\Pi_{k}(v)\right\| .
$$

Let $G$ be the set of $t>0$ such that $\operatorname{spec}\left(a_{t}^{k}\right) \cap\left[E_{t}-C_{q m} \cdot t, E_{t}+C_{q m} \cdot t\right]$ is nonempty. To prove the claim, it suffices to show that there exists $t_{0}>0$ so that $\left.G \cap\right] 0, t_{0}[=$ ] $0, t_{0}\left[\right.$ and for each $t<t_{0}$ the intersection $\operatorname{spec}\left(a_{t}^{k}\right) \cap\left[E_{t}-C_{q m} \cdot t, E_{t}+C_{q m} \cdot t\right]$ is a single point.

Let $B$ denote closure of the complement of $G$, namely the set of $t>0$ so that the distance from $E_{t}$ to the spectrum of $a_{t}^{k}$ is at least $C_{q m} \cdot t$. For each $\left.t \in B \cap\right] 0, t_{1}[$, we

\footnotetext{
11 This choice of $\beta$ is most probably not necessary but it will simplify the arguments in the appendix. In particular it implies that, on $[1, \beta]$ and for $\ell<k$, the Sturm-Liouville equations associated with $a_{t}^{\ell}$ have no turning point.
} 
apply a resolvent estimate to $(76)$ and obtain $\left\|\Pi_{k}\left(w_{t}\right)\right\| \leqslant\left\|w_{t}\right\| / 2$. Orthogonality then implies that for each $t \in \overline{B \cap}] 0, t_{1}[$, we have

$$
\left\|\Pi_{\ell<k} w_{t}\right\| \geqslant \frac{\sqrt{3}}{2} \cdot\left\|w_{t}\right\|
$$

By estimate (58) we have $\left\|w_{t}\right\| \sim\left\|u_{t}\right\|$, and so we can apply Proposition 7.12 to find $\kappa>0$ so that

$$
\dot{E}_{t} \geqslant \kappa \cdot \frac{\mathbb{1}_{B}(t)}{t}
$$

where $\mathbb{1}_{B}$ is the indicator function for $B$.

We first observe that (78) implies that 0 is a limit point of $G$. Indeed, since $\lim _{\epsilon \rightarrow 0} E_{\epsilon}=E_{0}$, the fundamental theorem of calculus implies that for each positive integer $n$, the limit

$$
\lim _{\epsilon \rightarrow 0} \int_{\epsilon}^{\frac{1}{n}} \dot{E}_{t} d t
$$

exists and is finite. Thus, by (78), the limit

$$
\limsup _{\epsilon \rightarrow 0} \int_{\epsilon}^{\frac{1}{n}} \frac{\mathbb{1}_{B}(t)}{t} d t
$$

is finite. Therefore, since $1 / t$ is not integrable on $] 0,1 / n]$, the set $] 0,1 / n] \cap G$ is nonempty for each $n$.

Next, we note that there exists a positive $t_{2} \leqslant t_{1}$ such that if $t<t_{2}$ and $t \in G$, then $\operatorname{spec}\left(a_{t}^{k}\right) \cap\left[E_{t}-C_{q m} \cdot t, E_{t}+C_{q m} \cdot t\right]$ consists of a single point. This is a consequence of the super-separation phenomenon described in Theorem 10.4 of HlrJdg11. We recall the exact statement here: Let $\left(s_{n}\right)_{n=1}^{\infty}$ be a sequence of positive numbers that tends to zero as $n$ tends to infinity. For each positive integer $n$, let $\lambda_{n}^{+}$and $\lambda_{n}^{-}$be distinct eigenvalues of $a_{s_{n}}^{k}$. If $\lambda_{n}^{ \pm}$tends to $(\pi k)^{2}$ as $n$ tends to $\infty$, then

$$
\lim _{n \rightarrow \infty} s_{n}^{-1} \cdot\left|\lambda_{n}^{+}-\lambda_{n}^{-}\right|=+\infty
$$

The set $\left.G \cap] 0, t_{2}\right]$ is a disjoint union of intervals. Since 0 is a limit point of $G$, to prove the theorem it suffices to show that the number of intervals is finite. Since the eigenbranches of $a_{t}^{k}$ are real-analytic for $t>0$, the intervals can be enumerated $I_{1}, I_{2}, I_{3} \ldots$ so that $\sup I_{j+1}<\inf I_{j}$ for each positive integer $j$. Because the spectrum of each $a_{t}^{k}$ is discrete, simple, and nonnegative, the eigenvalue branches of $a_{t}^{k}$ can be enumerated $\lambda^{1}, \lambda^{2}, \lambda^{3}, \ldots$ so that $\lambda_{t}^{\ell}<\lambda_{t}^{\ell+1}$ for each $t>0$. By superseparation, for each interval $I_{j}$ there exists a unique positive integer $\ell(j)$ so that for each $t \in I_{j}$ we have

$$
\operatorname{spec}\left(a_{t}^{k}\right) \cap\left[E_{t}-C_{q m} \cdot t, E_{t}+C_{q m} \cdot t\right]=\left\{\lambda_{t}^{\ell(j)}\right\} .
$$

To finish the proof of the theorem, it suffices to show that the function $j \mapsto \ell(j)$ is strictly decreasing. then

We claim that there exists $t_{3} \leqslant t_{2}$ such that if $t \in \partial G, t<t_{3}$, and $\lambda_{t}=E_{t} \pm C_{q m} \cdot t$,

$$
\dot{\lambda}_{t}<\dot{E}_{t}-C_{q m}<\dot{E}_{t}+C_{q m} .
$$

Indeed, if $t \in \partial G$, then $t \in B$ and so 78 gives that $\dot{E}_{t} \geqslant \kappa \cdot t$. Thus, if the claim were not true, then we would have a sequence $t_{n}$ tending to zero such that 


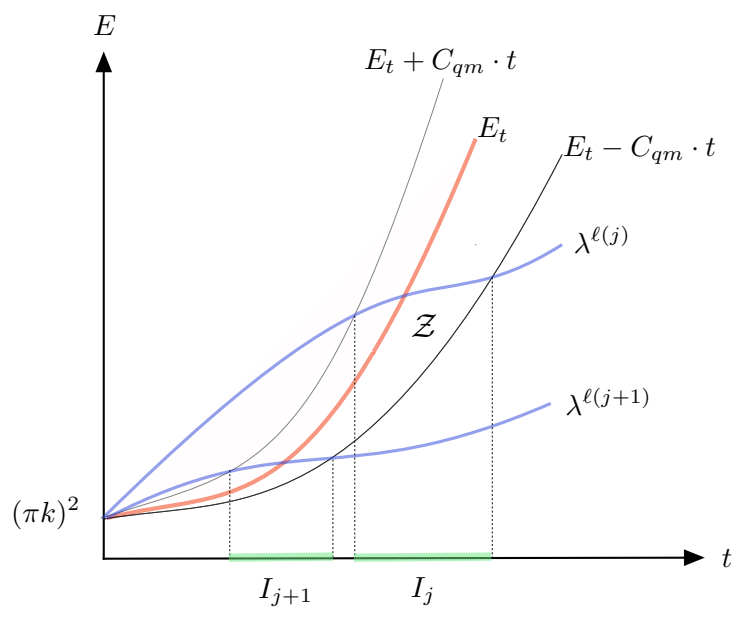

Figure 3. Let $\mathcal{Z}$ denote the set of $(t, E)$ such that $E \in\left[E_{t}-C_{q m}\right.$. $\left.t, E_{t}+C_{q m}\right]$. As $t$ decreases from $t_{3}$ to 0 , each eigenvalue branch $a_{t}^{k}$ that enters $\mathcal{Z}$ must enter from below, and if it exits $\mathcal{Z}$, then it must exit from above.

$t_{n} \cdot \dot{\lambda}_{t_{n}}$ would be bounded below by a positive constant $\kappa$. But this would contradict Lemma 8.4 below.

Let $a<t_{3}$ be the left endpoint of an interval $I_{j}$. Then $a \in \partial G$ and hence estimate 79 implies that there exists $\epsilon>0$ so that if $a-\epsilon<t<a$, then $\left.\lambda_{t}^{\ell(j)}\right\rangle$ $E_{t}+C_{q m} \cdot t$. Let $b$ be the infimum of $s<a$ such that for all $\left.t \in\right] b, a$ [ we have $\lambda^{\ell(j)}>E_{t}+C_{q m} \cdot t$. We can not have $b>0$. For then $b \in \partial G$ and estimate $79 p$ would give a contradiction. Therefore, $\lambda_{t}^{\ell(j)}>E_{t}+C_{q m} \cdot t$ for all $t<a$. See Figure 3. Thus, the branch $\lambda^{\ell(j+1)}$ must lie below the branch $\lambda^{\ell(j)}$. That is, $j \mapsto \ell(j)$ is strictly decreasing as desired.

Lemma 8.4. Let $k>0$ and let $t_{n}$ be a sequence converging to zero. For each $n \in$ $\mathbf{Z}^{+}$, let $t \mapsto \lambda_{n}(t)$ be a real-analytic eigenbranch of the family $a_{t}^{k}$. If $\lim _{n \rightarrow \infty} \lambda_{n}\left(t_{n}\right)=$ $(\pi k)^{2}$, then

$$
\lim _{n \rightarrow \infty} t_{n} \cdot \dot{\lambda}_{n}\left(t_{n}\right)=0
$$

Remark 8.5. One may replace the assumption in Lemma 8.4 that $t \mapsto \lambda_{n}(t)$ is realanalytic with the assumption that $t \mapsto \lambda_{n}(t)$ continuous. Indeed, for each $t>0$, the eigenvalue problem for $a_{t}^{k}$ corresponds to an eigenvalue problem for an ordinary differential equation, and hence the eigenvalues are simple. It follows that each continuous eigenvalue branch $t \mapsto \lambda_{n}(t)$ of the real-analytic family $a_{t}^{k}$ is necessarily real-analytic.

Remark 8.6. Lemma 8.4 is not a direct consequence of Lemma A.1 because the eigenvalue branch $\lambda_{n}$ may vary with $n$. By keeping track of the constants in the proof of Lemma A.1. one can produce a version that directly implies Lemma 8.4 . We prefer to give a direct proof here. 
Proof. For each $n$ we denote by $y \mapsto \psi_{n}(y)$ a unit norm eigenfunction of $a_{t_{n}}^{k}$ with eigenvalue $\lambda_{n}\left(t_{n}\right)$. By the standard variational formula and (35)

$$
\dot{\lambda}_{n}\left(t_{n}\right)=\dot{a}_{t_{n}}\left(\psi_{n}(y)\right)=2 t_{n} \cdot \int_{1}^{\infty}\left|\psi_{n}^{\prime}(y)\right|^{2} d y .
$$

Since $\psi_{n}$ is an eigenfunction of $a_{t_{n}}^{k}$ with eigenvalue $\lambda_{n}\left(t_{n}\right)$, using 35 , we have

$$
t_{n}^{2} \cdot \int_{1}^{\infty}\left|\psi_{n}^{\prime}(y)\right|^{2} d y=\int_{1}^{\infty}\left(\frac{\lambda_{n}\left(t_{n}\right)}{y^{2}}-(\pi k)^{2}\right)\left|\psi_{n}(y)\right|^{2} d y
$$

It suffices to show that the right hand side of 80 tends to zero as $n$ tends to infinity.

Let $\epsilon>0$. Since $\lambda_{n}\left(t_{n}\right)$ tends to $(\pi k)^{2}$, there exists $\delta>0$ so that if $|y-1|<\delta$, then $\left(\lambda_{n}\left(t_{n}\right) \cdot y^{-2}-(\pi k)^{2}\right)<\epsilon / 2$ and thus

$$
\int_{1}^{1+\delta}\left(\frac{\lambda}{y^{2}}-(\pi k)^{2}\right)\left|\psi_{n}(y)\right|^{2} d y \leqslant \frac{\epsilon}{2} \cdot \int_{1}^{1+\delta}\left|\psi_{n}(y)\right|^{2} d y .
$$

To estimate the remaining integral over $[1+\delta, \infty)$, we will apply a standard convexity estimate from the theory of ordinary differential equations. ${ }^{12}$ If $n$ is sufficiently large, $\lambda_{n}\left(t_{n}\right) /(\pi k)^{2}<(1+\delta / 4)^{2}$, and hence there exists $\eta>0$ so that if $y>z>1+\delta / 2$, then

$$
\left|\psi_{n}(y)\right|^{2} \leqslant\left|\psi_{n}(z)\right|^{2} \cdot \exp \left(-\frac{\eta}{t} \cdot(y-z)\right) .
$$

It follows that there exists $t_{0}$ so that if $t<t_{0}$, then

$$
\int_{1+\delta}^{\infty}\left|\psi_{n}(y)\right|^{2} d y \leqslant \frac{\epsilon}{4(\pi k)^{2}} \cdot \int_{1}^{\infty}\left|\psi_{n}(y)\right|^{2} d y
$$

Since $\left|\lambda_{n}\left(t_{n}\right) / y^{2}-(\pi k)^{2}\right| \leqslant(\pi k)^{2}$ for sufficiently large $n$, we may combine 82 with (81) to show that 80 is less than the given $\epsilon$ for sufficiently large $n$.

Notation. For the convenience of the reader, we recall the notation that is being used in the proof of the main theorem. We are considering an eigenbranch $\left(u_{t}, E_{t}\right)$ of $q_{t}$ such that $\lim _{t \rightarrow 0} E_{t}=k^{2} \pi^{2}>0$. We have chosen a compact interval $\left.I \subset\right](k-$ $1)^{2} \pi^{2},(k+1)^{2} \pi^{2}$ [ that contains $k^{2} \pi^{2}$ in its interior. We have set $w_{t}=P_{t}^{I} u_{t}$ where $P_{t}^{I}$ is the spectral projector on $I$ associated with $a_{t}$. Finally, $w_{t}^{k}$ is the orthogonal projection of $w_{t}$ onto $V_{k}$ so that there exists $v_{t}^{k} \in L^{2}\left((1,+\infty), y^{-2} d y\right)$ such that $w_{t}^{k}=v_{t}^{k} \otimes e_{k}$.

8.3. Crossings. In this subsection, we show that $\left\|w_{s}^{k}\right\|$ is smaller than $\left\|u_{s}\right\|$ for $s$ near a crossing time, a value of the parameter $t$ such that $E_{t}$ belongs to the spectrum of $a_{t}^{0}$. Then we show that there exists a sequence of crossing times $t_{n}$ and intervals of width $O\left(t^{\frac{8}{3}}\right)$ about the crossing times on which $\left\|w_{t}^{k}\right\|$ is smaller than $\rho\left\|u_{t}\right\|$ for some fixed $\rho$.

The proof of the first result depends on the analysis contained in Appendix B that provides estimates on the off-diagonal part of the quadratic form $b_{t}$ which, we recall, has been defined in (27) in such a way that it is the leading part of $q_{t}-a_{t}$ (see Proposition 6.2.

\footnotetext{
${ }^{12}$ See, for example, Lemma 6.3 in HlrJdg11.
} 
Proposition 8.7. Given $\rho<1$, there exists $\eta>0$ and $t_{0}>0$ such that if $t<t_{0}$ and

$$
\operatorname{dist}\left(E_{t}, \operatorname{spec}\left(a_{t}^{0}\right)\right) \leqslant \eta \cdot t^{\frac{5}{3}}
$$

then

$$
\left\|w_{t}^{k}\right\| \leqslant \rho \cdot\left\|u_{t}\right\| .
$$

Proof. Let $\psi_{t}^{0}$ be an eigenfunction of $a_{t}^{0}$ with eigenvalue $\lambda_{t}^{0}$ satisfying $\left|E_{t}-\lambda_{t}^{0}\right|<$ $\eta \cdot t^{\frac{5}{3}}$. We have

$$
\left(E_{t}-\lambda_{t}^{0}\right) \cdot\left\langle u_{t}, \psi_{t}^{0}\right\rangle=\left(a_{t}-q_{t}\right)\left(u_{t}, \psi_{t}^{0}\right)=t \cdot b_{t}\left(u_{t}, \psi_{t}^{0}\right)+O\left(t^{2}\right) \cdot\left\|u_{t}\right\| \cdot\left\|\psi_{t}^{0}\right\| .
$$

and hence

$$
\left|E_{t}-\lambda_{t}^{0}\right| \cdot\left|\left\langle u_{t}, \psi_{t}^{0}\right\rangle\right| \geqslant t \cdot\left|b_{t}\left(u_{t}, \psi_{t}^{0}\right)\right|-O\left(t^{2}\right) \cdot\left\|u_{t}\right\| \cdot\left\|\psi_{t}^{0}\right\| .
$$

In Appendix B Prop. B.1. we prove that there exists $\kappa>0$ so that

$$
\left|b_{t}\left(u_{t}, \psi_{t}^{0}\right)\right| \geqslant \kappa \cdot t^{\frac{2}{3}} \cdot\left(\left\|w_{t}^{k}\right\|-t^{\delta} \cdot\left\|u_{t}\right\|\right) \cdot\left\|\psi_{t}^{0}\right\| .
$$

Hence by applying the Cauchy-Schwarz inequality to the left hand side of (84), we find that

$$
\left|E_{t}-\lambda_{t}^{0}\right|\left\|u_{t}\right\| \cdot\left\|\psi_{t}^{0}\right\| \geqslant\left(\kappa \cdot t^{\frac{5}{3}} \cdot\left(\left\|w_{t}^{k}\right\|-t^{\delta} \cdot\left\|u_{t}\right\|\right)-O\left(t^{2}\right)\left\|u_{t}\right\|\right) \cdot\left\|\psi_{t}^{0}\right\| .
$$

Let $\eta=\rho \cdot \kappa / 2$, and use 83 to find that

$$
\frac{\rho}{2} \cdot\left\|u_{t}\right\| \geqslant\left\|w_{t}^{k}\right\|-O\left(t^{\delta}\right) \cdot\left\|u_{t}\right\|-O\left(t^{\frac{1}{3}}\right) \cdot\left\|u_{t}\right\| .
$$

The claim follows by choosing $t_{0}$ sufficiently small.

Proposition 8.8. For all $\eta>0$, let $\delta=\frac{\eta}{12(\pi k)^{2}}$. There exists $s_{0}>0$ such that if $s<s_{0}, E_{s} \in \operatorname{spec}\left(a_{s}^{0}\right)$, and $t \in\left[s, s+\delta \cdot s^{\frac{8}{3}}\right]$, then

$$
\operatorname{dist}\left(E_{t}, \operatorname{spec}\left(a_{t}^{0}\right)\right) \leqslant \eta \cdot s^{\frac{5}{3}} .
$$

Proof. Let $\lambda_{t}^{0}$ be the eigenvalue branch of $a_{t}^{0}$ such that $E_{s}=\lambda_{s}^{0}$. By Lemma 6.3. we have $\lambda_{t}^{0}=c \cdot t^{2}$ for some $c>0$, and hence $\dot{\lambda}_{t}^{0}=2 \cdot t^{-1} \cdot \lambda_{t}^{0}$. Using the fact that $a_{t}$ and $q_{t}$ are asymptotic and the fact that $\dot{a}_{t}$ is non negative, there exists a constant $C$ such that $\dot{E}_{t} \geqslant-C E_{t}$ for all sufficiently small $t$. Thus, for even smaller $t$ we obtain

$$
\frac{d}{d t} \ln \left(\frac{\lambda_{t}^{0}}{E_{t}}\right) \leqslant 3 \cdot t^{-1}
$$

Since $E_{s}=\lambda_{s}$, integration over $[s, t]$ and exponentiation gives

$$
\frac{\lambda_{t}^{0}}{E_{t}} \leqslant\left(\frac{t}{s}\right)^{3}
$$

If $t \leqslant s+\delta \cdot s^{\frac{8}{3}}$, then

$$
\left(\frac{t}{s}\right)^{3} \leqslant\left(1+\delta \cdot s^{\frac{5}{3}}\right)^{3} \leqslant 1+4 \cdot \delta \cdot s^{\frac{5}{3}}
$$

where the last inequality holds for $s \leqslant s_{1}=(2 \delta)^{-\frac{3}{5}}$. By combining this with 85, one finds that for $t \in\left[s, s+\delta \cdot s^{\frac{8}{3}}\right]$, we have

$$
\lambda_{t}^{0}-E_{t} \leqslant E_{t} \cdot 4 \cdot \delta \cdot s^{\frac{5}{3}}
$$


Using Lemma 7.13 , and $\dot{\lambda} \geqslant 0$, we have that

$$
\frac{d}{d t} \ln \left(\frac{E_{t}}{\lambda_{t}^{0}}\right) \leqslant 3 \cdot t^{-1}
$$

An argument similar to the one above gives that

$$
E_{t}-\lambda_{t}^{0} \leqslant \lambda_{t}^{0} \cdot 4 \cdot \delta \cdot s^{\frac{5}{3}}
$$

for $t \in\left[s, s+\delta \cdot s^{\frac{8}{3}}\right]$.

Since by assumption $\lim _{t \rightarrow 0} E_{t}=(\pi k)^{2}$, there exists $s_{2}$ so that if $t<s_{2}+\delta \cdot s_{2}^{\frac{8}{3}}$, then $E_{t} \leqslant 2 \cdot(\pi k)^{2}$. Therefore, by 86 , we have that $\left\{\lambda_{t}^{0} \mid s \leqslant t \leqslant s+\delta \cdot s^{\frac{8}{3}}\right\}$ is bounded above by $3(\pi k)^{2}$ for $s<s_{0}=\min \left\{s_{1}, s_{2}\right\}$. In sum, if $s<s_{0}$ and $t \in\left[s, s+\delta \cdot s^{\frac{8}{3}}\right]$, then

$$
\left|\lambda_{t}^{0}-E_{t}\right| \leqslant 12(\pi k)^{2} \cdot \delta \cdot s^{\frac{5}{3}} \leqslant \eta \cdot s^{\frac{5}{3}},
$$

by the choice of $\delta$.

We wish to estimate from below the size of the set of $t$ for which 83 holds true. This is accomplished by the following proposition.

Proposition 8.9. Let $\delta>0$. There is a sequence $t_{n}$ of crossing times such that

$$
\lim _{n \rightarrow \infty} n \cdot t_{n}=k \cdot \ln (\beta) .
$$

and if $n \neq m$ are large enough, then the intervals $\left[t_{n}, t_{n}+\delta \cdot t_{n}^{\frac{8}{3}}\right]$ and $\left[t_{m}, t_{m}+\delta \cdot t_{m}^{\frac{8}{3}}\right]$ are disjoint.

Proof. By Lemma A.1. there exists, $\nu^{*}>0$ so that $\lambda_{t}^{*}=(\pi k)^{2}+\nu^{*} \cdot t^{\frac{2}{3}}+O\left(t^{\frac{4}{3}}\right)$. It also follows from Proposition 8.3 that there exists $M$ so that

$$
(\pi k)^{2}+\nu^{*} \cdot t^{\frac{2}{3}}-M \cdot t<E_{t}<(\pi k)^{2}+\nu^{*} \cdot t^{\frac{2}{3}}+M \cdot t
$$

for sufficiently small $t$. By Lemma 6.3 the eigenvalues of $a_{t}^{0}$ have the form $c_{n} \cdot t^{2}$ where $c_{n}=\left(1 / 4+r_{n}^{2}\right)$ and $r_{n}$ is the increasing sequence of positive solutions to the equation $2 r=\tan (r \ln (\beta))$. Standard asymptotic analysis shows that

$$
r_{n}=\frac{n \pi+\frac{\pi}{2}}{\ln (\beta)}+o(1) \text {. }
$$

Fix $0<\nu_{0}^{-}<\nu^{*}<\nu_{0}^{+}$. For each $\nu \in\left[\nu_{0}^{-}, \nu_{0}^{+}\right]$and each $n \in \mathbb{Z}$, there exists a unique $t_{n}^{\nu} \in \mathbb{R}^{+}$so that

$$
c_{n} \cdot\left(t_{n}^{\nu}\right)^{2}=(\pi k)^{2}+\nu \cdot\left(t_{n}^{\nu}\right)^{\frac{2}{3}} .
$$

We drop the dependence in $\nu$ from the notation for a moment. If we set $x_{n}=c_{n}^{-\frac{1}{6}}$ and $y_{n}=c_{n}^{\frac{1}{6}} \cdot t_{n}^{\frac{1}{3}}$ then 911 becomes

$$
y_{n}^{6}=(\pi \cdot k)^{2}+\nu \cdot x_{n}^{2} \cdot y_{n}^{2} .
$$

The analytic (polynomial) function $F$ defined by $F(x, y)=y^{6}-(\pi \cdot k)^{2}-\nu \cdot x^{2} \cdot y^{2}$ satisfies $F\left(0,(\pi k)^{\frac{2}{3}}\right)=0$ and $\partial_{y} F\left(0,(\pi k)^{\frac{2}{3}}\right) \neq 0$. Thus, by the analytic implicit function theorem, for $x$ near 0 , there exist a unique analytic function $Y(x)$ so that

$$
Y(x)^{6}=(\pi \cdot k)^{2}+\nu \cdot x^{2} \cdot Y(x)^{2} .
$$




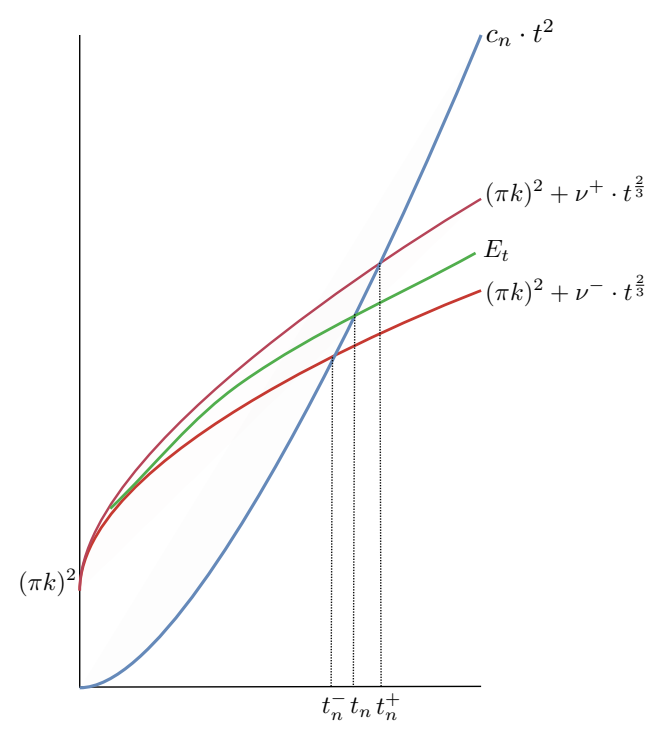

Figure 4 . The crossing $t_{n}$.

By inspecting the first few coefficients in the Taylor expansion of $Y^{3}$, we find that

$$
Y(x)^{3}=\pi \cdot k+\frac{\nu}{2 \cdot(\pi k)^{\frac{1}{3}}} \cdot x^{2}+O\left(x^{3}\right) .
$$

Thus, since $\lim _{n \rightarrow \infty} x_{n}=0$, and $t_{n}=c_{n}^{-\frac{1}{2}} \cdot Y^{3}\left(c_{n}^{-\frac{1}{2}}\right)$ we find that

$$
t_{n}^{\nu}=(\pi k) \cdot c_{n}^{-\frac{1}{2}}+\tau \cdot c_{n}^{-\frac{5}{6}}+O\left(c_{n}^{-1}\right) .
$$

where $\tau=\nu \cdot(\pi k)^{\frac{1}{3}} / 2$.

Choose $\epsilon>0$ so that if $\nu^{ \pm}=\nu^{*} \pm \epsilon$, then $\nu_{0}^{-} \leqslant \nu^{-}<\nu^{*}<\nu^{+} \leqslant \nu_{0}^{+}$. Define $t_{n}^{ \pm}=t_{n}^{ \pm \nu}$. By applying the intermediate value theorem to $\lambda_{t}-E_{t}$, there exists $\left.t_{n} \in\right] t_{n}^{-}, t_{n}^{+}$[ so that $E_{t_{n}}=\lambda_{t_{n}}$. See Figure 4. Since $c_{n}$ is increasing to infinity, the sequence $t_{n}$ is decreasing to zero.

Moreover, since $\nu^{ \pm}=\nu^{*} \pm \varepsilon$

$$
t_{n}=(\pi k) \cdot c_{n}^{-\frac{1}{2}}+\tau^{*} \cdot c_{n}^{-\frac{5}{6}}+o\left(c_{n}^{-\frac{5}{6}}\right) .
$$

where $\tau^{*}=\nu^{*} \cdot(\pi k)^{\frac{1}{3}} / 2$. From 90 we have

$$
c_{n}^{-1}=\frac{\sigma^{2}}{n^{2}} \cdot\left(1+O\left(\frac{1}{n}\right)\right)
$$

where $\sigma=\ln (\beta) / \pi$. By substituting this into 92 and 93 we find that

$$
t_{n}^{ \pm}=(\pi k) \cdot n^{-1}+\tau \cdot \sigma^{\frac{5}{3}} \cdot n^{-\frac{5}{3}}+O_{ \pm}\left(n^{-2}\right)
$$

and

$$
t_{n}=(\pi k) \cdot n^{-1}+\tau^{*} \cdot \sigma^{\frac{5}{3}} \cdot n^{-\frac{5}{3}}+o\left(n^{-\frac{5}{3}}\right) .
$$


The first claim follows. Moreover, since $\nu^{ \pm}=\nu^{*} \pm \varepsilon$, we have

$$
\begin{aligned}
t_{n}^{+}-t_{n} & \sim \varepsilon \cdot \frac{(\pi k)^{\frac{1}{3}}}{2} \cdot n^{-\frac{5}{3}} \\
t_{n}-t_{n}^{-} & \sim \varepsilon \cdot \frac{(\pi k)^{\frac{1}{3}}}{2} \cdot n^{-\frac{5}{3}} \\
t_{n}^{-}-t_{n+1}^{+} & \sim \varepsilon \cdot(\pi k)^{\frac{1}{3}} \cdot n^{-\frac{5}{3}} \\
t_{n}^{\frac{8}{3}} & =O\left(n^{-\frac{8}{3}}\right) .
\end{aligned}
$$

It follows that, for all sufficiently large $n$, we have $\left[t_{n}, t_{n}+\delta \cdot t_{n}^{\frac{8}{3}}\right] \subset\left[t_{n}^{-}, t_{n}^{+}\right]$. Since the intervals $\left\{\left[t_{n}^{-}, t_{n}^{+}\right]\right\}$are disjoint, the claim is proven.

8.4. Relative variation and the contradiction. In this section we derive the desired contradiction. In particular, we prove the following.

Theorem 8.10. Suppose that $E_{t}$ is a cusp form eigenvalue branch with a positive limit. If $\lambda_{t}^{*}$ is the eigenvalue branch of $a_{t}$ that satisfies (75), then there exists $t_{0}>0$ and $c>0$ so that if $t<t_{0}$, then

$$
E_{t}-\lambda_{t}^{*}>c \cdot t^{\frac{2}{3}}
$$

The proof will consist of two types of lower estimates. The first depends on the fact that near each crossing the 'relative variation' $\dot{E}_{t}-\dot{\lambda}_{t}^{*}$ is at least of order $O\left(t^{-1}\right)$. The second shows that away from the crossings the relative variation is not too negative.

Define

$$
\left.K(t, \rho)=\{s \in] 0, t]\|\| w_{s}^{k}\|\leqslant \rho \cdot\| u_{s} \|\right\} .
$$

If $\rho<1$, then it follows from Proposition 7.12 that there exists $\kappa>0$ so that for $s \in K(t, \rho)$ we have

$$
\dot{E}_{s} \geqslant \kappa \cdot s^{-1} .
$$

Hence, since $\dot{\lambda}_{t}^{*}=O\left(t^{-\frac{1}{3}}\right)$, there exists $t^{*}>0$ so that if $t<t^{*}$ and $\rho<1$, then

$$
\dot{E}_{s}-\dot{\lambda}_{s}^{*} \geqslant \frac{\kappa}{2} \cdot s^{-1} \text {. }
$$

for each $s \in K(t, \rho)$. We will integrate this estimate near the crossings to obtain the following.

Lemma 8.11. For each $\rho<1$, there exists $t_{0}>0$ and $\gamma(\rho)>0$ so that for each $t<t_{0}$, we have

$$
\int_{K(t, \rho)}\left(\dot{E}_{s}-\dot{\lambda}_{s}\right) d s \geqslant \gamma(\rho) \cdot t^{\frac{2}{3}} .
$$

Proof. By (95) the integrand is positive on $K(t, \rho)$, it suffices to show the same estimate holds for a subset $G$ of $K(t, \rho)$.

To define this subset, we first combine Proposition 8.7, Proposition 8.8, and Proposition 8.9 to find $\delta>0, N^{\prime} \geqslant 2$, and a monotone sequence $\left\{t_{n}\right\}$ so that for each $n$

$$
I_{n, \delta}=\left[t_{n}, t_{n}+\delta \cdot t_{n}^{\frac{8}{3}}\right]
$$

belongs to $K(1 / 2, \rho)$, the intervals $I_{n}$ and $I_{n+1}$ are disjoint, and for each $n \geqslant N^{\prime}$

$$
\frac{\tau}{2 n} \leqslant t_{n} \leqslant \frac{2 \tau}{n}
$$


LUC HILLAIRET AND CHRIS JUDGE

where $\tau=k \cdot \ln (\beta)$. The subset $G$ will be defined as a union of $I_{n}$ over sufficiently large $n$.

We have $\int_{I_{n, \delta}} s^{-1} d s=\ln \left(1+\delta \cdot t_{n}^{\frac{5}{3}}\right)$ and hence there exists $N^{*} \geqslant N^{\prime}$ so that if $n \geqslant N^{*}$ we have

$$
\int_{I_{n, \delta}} s^{-1} d s \geqslant \frac{\delta}{2} \cdot t_{n}^{\frac{5}{3}}
$$

Thus, from 96 we find that if $N \geqslant N^{*}$, then

$$
\left(\frac{2}{\tau}\right)^{\frac{5}{3}} \sum_{n \geqslant N+2} t_{n}^{\frac{5}{3}} \geqslant \sum_{n \geqslant N+2} n^{-\frac{5}{3}} \geqslant \int_{N+2}^{\infty} x^{-\frac{5}{3}} d x=(N+2)^{-\frac{2}{3}} \geqslant\left(\frac{t_{N}}{4 \tau}\right)^{\frac{2}{3}} .
$$

Since the intervals $I_{n, \delta}$ are disjoint, by combining (95), (97), and $(98)$, we find that

$$
\int_{G_{N}}\left(\dot{E}_{s}-\dot{\lambda}_{s}^{*}\right) d s \geqslant \gamma \cdot t_{N}^{\frac{2}{3}}
$$

where $\gamma=\kappa \cdot \delta \cdot \tau \cdot 2^{-\frac{16}{3}}$ and

$$
G_{N}:=\bigcup_{n \geqslant N+2} I_{n, \delta}
$$

Let $t_{0}=t_{N^{*}}$. If $t<t_{0}$, then $t \in\left[t_{N+1}, t_{N}\right]$ for some $N \geqslant N^{*}$. We have $t_{N+2}+t_{N+2}^{\frac{8}{3}} \leqslant t_{N+1} \leqslant t$ and hence $G_{N} \subset K(t, \rho)$ and $t_{N}^{\frac{2}{3}} \geqslant t^{\frac{2}{3}}$. Therefore, 99 implies the claim.

To bound the relative variation on the complement of $K(t, \rho)$, we will use the following.

Proposition 8.12. There exists $C$ and $t_{0}>0$ such that, if $t<t_{0}$, then

$$
\dot{E}_{t} \geqslant \frac{\left\|w_{t}^{k}\right\|^{2}}{\left\|u_{t}\right\|^{2}} \cdot \dot{\lambda}_{t}^{*}-C \cdot t^{-\frac{1}{9}} .
$$

Proof. By arguing as in 70 we have

$$
\dot{E}_{t} \cdot\left\|u_{t}\right\|^{2} \geqslant \dot{a}_{t}\left(w_{t}^{k}\right)-O\left(\left\|u_{t}\right\|^{2}\right) .
$$

Let $w_{t}^{*}$ denote the orthogonal projection of $w_{t}^{k}$ onto the eigenfunction branch of $a_{t}$ that corresponds to $\lambda_{t}^{*}$ from Theorem 8.3 . Let $w_{t}^{\perp}:=w_{t}^{k}-w_{t}^{*}$. For $\diamond=k, *, \perp$, we define $v_{t}^{\diamond}$ so that $w_{t}^{\diamond}=v_{t}^{\diamond} \otimes e_{k}$. Observe that by definition, $v_{t}^{*}$ is an eigenfunction of $a_{t}^{k}$ with eigenvalue $\lambda_{t}^{*}$.

Using the Cauchy-Schwarz inequality and the nonnegativity of $\dot{a}_{t}$ we have

$$
\dot{a}_{t}\left(w_{t}^{k}\right)=\dot{a}_{t}^{k}\left(v_{t}^{k}\right) \geqslant \dot{a}_{t}^{k}\left(v_{t}^{*}\right)-2 \cdot\left|\dot{a}_{t}^{k}\left(v_{t}^{*}, v_{t}^{\perp}\right)\right| .
$$

Since $v_{t}^{*}$ is an eigenfunction, we have $\dot{a}_{t}^{k}\left(v_{t}^{*}\right)=\dot{\lambda}^{*} \cdot\left\|v_{t}^{*}\right\|^{2}$. Using (35) and the fact that $v_{t}^{*}$ is an eigenfunction that is orthogonal to $v_{t}^{\perp}$, we find that

$$
\begin{aligned}
\dot{a}_{t}^{k}\left(v_{t}^{*}, v_{t}^{\perp}\right) & =2 t^{-1} \cdot\left(a_{t}^{k}\left(v_{t}^{*}, v_{t}^{\perp}\right)-(\pi k)^{2} \int_{1}^{\infty} v_{t}^{*}(y) \cdot v_{t}^{\perp}(y) d y\right) \\
& =-2(\pi k)^{2} \cdot t^{-1} \int_{1}^{\infty} v_{t}^{*}(y) \cdot v_{t}^{\perp}(y) d y .
\end{aligned}
$$


Since $\left\langle v_{t}^{*}, v_{t}^{\perp}\right\rangle=0$, we have

$$
\int_{1}^{\infty} v_{t}^{*} \cdot v_{t}^{\perp} d y=\int_{1}^{\infty} v_{t}^{*} \cdot v_{t}^{\perp} \cdot\left(1-y^{-2}\right) d y
$$

The large $y$ asymptotics of $v_{t}^{*}$ and $v_{t}^{\perp}$ can be analysed using the same methods as in Appendix $\mathrm{B}$ for $v_{t}^{k}$. We thus define $r_{t}^{\diamond}$, for $\diamond=k, *, \perp$, by

$$
r_{t}^{\diamond}=t^{2}\left(v_{t}^{\diamond}\right)^{\prime \prime}+\left(\frac{E_{t}}{y^{2}}-k^{2} \pi^{2}\right) v_{t}^{\diamond}
$$

so that Prop. B.7 gives

$\int_{1+2 t^{\alpha}}^{\infty}\left|v_{t}^{\diamond}(y)\right|^{2} d y \leqslant C \cdot\left(t^{-2 \alpha} \cdot \int_{1}^{\infty}\left|r_{t}^{\diamond}\right|^{2}+\exp \left(-t^{\frac{3 \alpha-2}{2}}\right) \cdot \int_{1+t^{\alpha}}^{\infty}\left|v_{t}^{\diamond}(y)\right|^{2} y^{-2} d y\right)$.

We can now estimate $r_{t}^{\diamond}$ with the same techniques as in LemmaB.5: we test again a smooth function $\phi$ to obtain

$$
\int_{1}^{\infty} r_{t}^{\diamond}(y) \phi(y)=-a_{t}^{k}\left(v_{t}^{\diamond}, \phi\right)+E_{t} \cdot\left\langle v_{t}^{\diamond}, \phi\right\rangle
$$

We now observe that $v_{t}^{\diamond} \otimes e_{k}=P_{t}^{\diamond} w_{t}$ where $P_{t}^{\diamond}$ is some spectral projector associated with $a_{t}$. Arguing as in Corollary 7.8 , we thus obtain

$$
\left|\int_{1}^{\infty} r_{t}^{\diamond}(y) \phi(y) d y\right| \leqslant C^{\prime} \cdot t \cdot\left\|w_{t}\right\| \cdot\|\phi\| .
$$

This now implies (see the proof of Lemma B.5

$$
\int_{1}^{\infty}\left|r_{t}^{\diamond}(y)\right|^{2} d y \leqslant C \cdot t^{2} \cdot\left\|w_{t}\right\|^{2}
$$

We plug this estimate into (104) (see also the proof of Corollary B.8) to obtain that, for each $\alpha<\frac{2}{3}$, there exists a constant $C$ so that for sufficiently small $t$

$$
\int_{1+2 t^{\alpha}}^{\infty}\left|v_{t}^{*}\right|^{2} d y \leqslant C \cdot t^{2-2 \alpha} \cdot\left\|w_{t}\right\|^{2}
$$

and

$$
\int_{1+2 t^{\alpha}}^{\infty}\left|v_{t}^{\perp}\right|^{2} d y \leqslant C \cdot t^{2-2 \alpha} \cdot\left\|w_{t}\right\|^{2} .
$$

If $y \leqslant 1+2 t^{\alpha}$, then $\left(1-y^{-2}\right) \leqslant 4 t^{\alpha}$ for sufficiently small $t$. Therefore, by splitting the domain of integration into $\left[1,1+2 t^{\alpha}\right]$ and $\left[1+2 t^{\alpha}, \infty[\right.$ and using the CauchySchwarz inequality, we find that

$$
\left|\int_{1}^{\infty} v_{t}^{*} \cdot v_{t}^{\perp} d y\right| \leqslant 5 \cdot t^{\alpha} \cdot\left\|v_{t}^{*}\right\| \cdot\left\|v_{t}^{\perp}\right\|+C \cdot t^{2-2 \alpha} \cdot\left\|w_{t}\right\|^{2}
$$

for sufficiently small $t$.

We claim that $\left\|v_{t}^{\perp}\right\|=O\left(t^{\frac{1}{3}}\right) \cdot\left\|w_{t}\right\|$. Indeed, by applying Lemma 7.7 with $v \in V_{k}$, we have

$$
\left|a_{t}^{k}\left(v_{t}^{k}, v\right)-E \cdot\left\langle v_{t}^{k}, v\right\rangle\right| \leqslant C \cdot t \cdot\left\|w_{t}\right\| \cdot\|v\|
$$

for some constant $C$. Thus, since the eigenvalue $\lambda^{*}$ satisfies $\left|E_{t}-\lambda^{*}\right|<C^{\prime} \cdot t$ we find that

$$
\left|a_{t}^{k}\left(v_{t}^{\perp}, v\right)-E \cdot\left\langle v_{t}^{\perp}, v\right\rangle\right| \leqslant 2 C \cdot t \cdot\left\|w_{t}\right\| \cdot\|v\| \cdot
$$


By definition, $v_{t}^{\perp}$ is a spectral projection onto eigenspaces of $a_{t}^{k}$ whose associated eigenvalues are distinct from $\lambda^{*}$. By Lemma A.1 there exists $\delta>0$ so that such eigenvalues differ from $\lambda^{*}$ by at least $\delta \cdot t^{\frac{2}{3}}$. Because of 106, we can thus apply a resolvent estimate (e.g. Lemma 2.1 [HlrJdg11]) to find that

$$
\left\|v_{t}^{\perp}\right\| \leqslant \frac{2 C}{\delta} \cdot t^{\frac{1}{3}} \cdot\left\|w_{t}\right\|
$$

By substituting 107 into 105 and setting $\alpha=5 / 9$, we find a constant $C^{\prime}$ so that

$$
\left|\int_{1}^{\infty} v_{t}^{*} \cdot v_{t}^{\perp} d y\right| \leqslant C^{\prime} \cdot t^{\frac{8}{9}} \cdot\left\|w_{t}\right\|^{2} .
$$

By combining this estimate with (103), 102), and 101), we obtain a constant $C^{\prime \prime}$ so that

$$
\dot{E}_{t} \cdot\left\|u_{t}\right\|^{2} \geqslant \dot{\lambda}^{*} \cdot\left\|w_{t}^{*}\right\|^{2}-2 C^{\prime \prime} \cdot t^{-\frac{1}{9}} \cdot\left\|w_{t}\right\|^{2} .
$$

By orthogonality $\left\|w_{t}^{*}\right\|^{2}=\left\|w_{t}^{k}\right\|^{2}-\left\|w_{t}^{\perp}\right\|^{2}$, and hence by (107) and Lemma A.1. we have a constant $C^{\prime \prime \prime}$ so that

$$
\dot{\lambda}^{*} \cdot\left\|w_{t}^{*}\right\|^{2}=\dot{\lambda}^{*} \cdot\left\|w_{t}^{k}\right\|^{2}-C^{\prime \prime \prime} \cdot t^{\frac{1}{3}} \cdot\left\|w_{t}\right\|^{2} .
$$

The desired result follows.

Corollary 8.13. There exists $C^{\prime}$ such that for each $\left.\rho \in\right] 0,1\left[\right.$, there exists $t_{0}>0$ such that if $0<t<t_{0}$, then

$$
\int_{[0, t] \backslash K(t, \rho)}\left(\dot{E}_{s}-\dot{\lambda}_{s}^{*}\right) d s \geqslant C^{\prime} \cdot\left(\rho^{2}-1\right) \cdot t^{\frac{2}{3}} .
$$

Proof. By definition, if $s \in[0, t] \backslash K(t, \rho)$, then $\left\|w_{t}^{k}\right\|^{2} /\left\|u_{t}\right\|^{2} \geqslant \rho^{2}$ and hence from Prop. 8.12 , we find that

$$
\dot{E}_{t}-\dot{\lambda}_{t}^{*} \geqslant\left(\rho^{2}-1\right) \cdot \dot{\lambda}_{t}^{*}-C \cdot t^{-\frac{1}{9}} .
$$

By using Lemma A.1. we find $C^{\prime}$ and $t_{0}$ so that for $t<t_{0}$

$$
\dot{E}_{t}-\dot{\lambda}_{t}^{*} \geqslant \frac{2}{3} \cdot C^{\prime} \cdot\left(\rho^{2}-1\right) \cdot t^{-\frac{1}{3}}
$$

The claim follows from integration.

Finally, we use Lemma 8.11 and Corollary 8.13 to prove Theorem 8.10 . This will complete the proof of the main theorem.

Proof of Theorem 8.10. Apply Lemma 8.11 with $\rho=1 / 2$. Then apply Corollary 8.13 with $\rho=\rho_{0} \geqslant 1 / 2$ such that

$$
C^{\prime} \cdot \frac{\rho_{0}^{2}-1}{2} \geqslant-\frac{1}{2} \cdot \gamma\left(\frac{1}{2}\right)
$$

Since $s \mapsto \dot{E}_{s}-\dot{\lambda}_{s}^{*}$ is positive on $K\left(t, \rho_{0}\right) \supset K\left(t, \frac{1}{2}\right)$, we find that

$$
\int_{0}^{t}\left(\dot{E}_{s}-\dot{\lambda}_{s}^{*}\right) d s \geqslant \frac{1}{2} \cdot \gamma\left(\frac{1}{2}\right) \cdot t^{\frac{2}{3}}
$$

Since $\lim _{t \rightarrow 0} E_{t}-\lambda_{t}^{*}=0$, we have the desired conclusion. 


\section{Appendix A. Eigenvalue Branches of $a_{t}^{\ell}$}

In this appendix, we compute the asymptotics of each real-analytic eigenvalue branch of $a_{t}^{\ell}$ for each $\ell \in \mathbb{Z}^{+}$.

Proposition A.1. Let $\ell \in \mathbb{Z}^{+}$and let $t \mapsto \lambda_{t}$ be a real-analytic eigenvalue branch of $a_{t}^{\ell}$ for $t>0$. Then

$$
\lambda_{t}=(\ell \pi)^{2}+a \cdot t^{\frac{2}{3}}+O\left(t^{\frac{4}{3}}\right) .
$$

where $a=\left(2(\pi \ell)^{2}\right)^{\frac{2}{3}} \cdot(-\zeta)$ and $\zeta$ is a zero of the derivative of the Airy function $A_{-}$defined in (161). Moreover,

$$
\lim _{t \rightarrow 0^{+}} \dot{\lambda}_{t} \cdot t^{\frac{1}{3}}=\frac{2}{3} \cdot a .
$$

To prove Proposition A.1], we will first transform the eigenvalue problem into an eigenvalue problem that is easier to analyse. If $v$ is an eigenfunction of $a_{t}^{\ell}$ with respect to $\|\cdot\|$ with eigenvalue $\lambda$, then for each $w \in C_{0}^{\infty}([0, \infty[)$ and $t>0$, we have

$$
t^{2} \int_{1}^{\infty} v^{\prime} \cdot w^{\prime} d y+\mu \int_{1}^{\infty} v \cdot w d y=\lambda \int_{1}^{\infty} \frac{v \cdot w}{y^{2}} d y
$$

where we have set $\mu=\ell^{2} \pi^{2}$.

Hence

$$
t^{2} \int_{1}^{\infty} v^{\prime} \cdot w^{\prime} d y+\mu \int_{1}^{\infty} \frac{(y-1) \cdot(y+1)}{y^{2}} \cdot v \cdot w d y=(\lambda-\mu) \int_{1}^{\infty} \frac{v \cdot w}{y^{2}} d y .
$$

By making the change of variable $y=t^{\frac{2}{3}} \cdot x+1$, letting $\bar{v}(x)=v\left(t^{\frac{2}{3}} \cdot x+1\right)$ and $\bar{w}(x)=w\left(t^{\frac{2}{3}} \cdot x+1\right)$, and dividing by $t^{\frac{4}{3}}$, we find that

$$
\int_{0}^{\infty} \bar{v}_{t}^{\prime} \cdot \bar{w}^{\prime} d x+\mu \int_{0}^{\infty} x \cdot g\left(t^{\frac{2}{3}} \cdot x\right) \cdot \bar{v}_{t} \cdot \bar{w} d x=t^{-\frac{2}{3}} \cdot(\lambda-\mu) \int_{0}^{\infty} f\left(t^{\frac{2}{3}} \cdot x\right) \cdot \bar{v}_{t} \cdot \bar{w} d x .
$$

where

and

$$
f(z)=\frac{1}{(z+1)^{2}}
$$

$$
g(z)=\frac{z+2}{(z+1)^{2}}
$$

This leads us to set $s=t^{\frac{1}{3}}$ and define for each $w \in C_{0}^{\infty}([0, \infty[)$ the quadratic forms

and

$$
\mathcal{A}_{s}(w)=\int_{0}^{\infty}\left(w^{\prime}\right)^{2} d x+\mu \int_{0}^{\infty} x \cdot g\left(s^{2} \cdot x\right) \cdot w^{2} d x
$$

$$
\mathcal{N}_{s}(w)=\int_{0}^{\infty} f\left(s^{2} \cdot x\right) \cdot w^{2} d x
$$

Define $\mathcal{H}:=L^{2}\left([0, \infty), \frac{1}{x^{2}+1} d x\right)$ and $\mathcal{D}:=H^{1}([0, \infty)$ ) (i.e. the set of functions $u \in L^{2}([0, \infty))$ such that the distributional derivative also is in $\left.L^{2}\right)$.

Then for each $s>0$, the form $\mathcal{N}_{s}$ is a bounded quadratic form on $\mathcal{H}$ and $\mathcal{A}_{s}$ is a closed quadratic form on $\mathcal{H}$ with domain $\mathcal{D}$.

Since $w \mapsto \bar{w}$ maps bijectively $C_{0}^{\infty}([1, \infty])$ onto $C_{0}^{\infty}([0, \infty])$, the function $\bar{v}$ is an eigenfunction of $\mathcal{A}_{s}$ with respect to $\mathcal{N}_{s}$ with eigenvalue $\nu=s^{-2} \cdot(\lambda-\mu)$. 
It follows from the perturbation theory of generalized eigenvalue problems (see $\S$ VII.6 in [Kato] that the eigenvalues of $\mathcal{A}_{s}$ with respect to $\mathcal{N}_{s}$ can be organized into real-analytic eigenvalue branches for $s>0$

Since the generalized eigenvalue problem $\mathcal{A}_{s}(u, v)=\nu \cdot \mathcal{N}_{s}(u, v)$ corresponds to a Sturm-Liouville problem with Neumann condition at $x=0$, the eigenspaces are 1-dimensional. Hence, we may enumerate the real-analytic eigenvalue branches $\nu_{s}^{i}$ so that for each $i \geqslant 0$ and $s>0$ we have

$$
\nu_{s}^{i}<\nu_{s}^{i+1} .
$$

Lemma A.2. For each $i$, there exists $s_{0}>0$ and $C$ so that if $s<s_{0}$ then

$$
\left|\dot{\nu}_{s}^{i}\right| \leqslant C \cdot s \text {. }
$$

In particular, there exists a so that for small $s>0$

$$
\nu_{s}^{i}=a+O\left(s^{2}\right) .
$$

Moreover, $-a /(2 \mu)^{\frac{2}{3}}$ is a zero of the derivative of the Airy function $A_{-}$.

Proof. First, we show that each $\nu_{i}^{s}$ is bounded. To this end, define

$$
\mathcal{B}(v)=\int_{0}^{\infty}\left(v^{\prime}(x)\right)^{2}+2 \mu \cdot x \cdot v(x)^{2} d x .
$$

Since $g$ is bounded above by 2 , we have $\mathcal{A}_{s}(v) \leqslant \mathcal{B}(v)$ for each $s>0$ and $v \in$ $C_{0}^{\infty}\left(\left[0, \infty[)\right.\right.$. Note that for each $s \leqslant 1$, we have $\mathcal{N}_{s}(v) \geqslant \mathcal{N}_{1}(x)$ and hence

$$
\frac{\mathcal{A}_{s}(v)}{\mathcal{N}_{s}(v)} \leqslant \frac{\mathcal{B}(v)}{\mathcal{N}_{1}(v)}
$$

Integration by parts shows that the eigenfunctions of $\mathcal{B}$ with respect to $\mathcal{N}_{1}$ are solutions to the Sturm-Liouville problem

$$
-v^{\prime \prime}(x)+2 \mu \cdot x \cdot v(x)=\frac{v(x)}{(1+x)^{2}} .
$$

Standard convexity estimates on solutions to ordinary differential equations imply that each eigenfunction belongs to the domain $\mathcal{D}$ of $\mathcal{A}_{s}$ for each $s>0$. In particular, the sum of the first $i$ eigenspaces of $\mathcal{B}$ with respect to $\mathcal{N}_{1}$ belongs to $\mathcal{D}$.

Therefore, using (113), the minimax principle, and 110 we find that $\nu_{s}^{i}$ is bounded by the $i^{\text {th }}$ eigenvalue of $\mathcal{B}$ with respect to $\mathcal{N}_{1}$.

In the remainder of the argument we drop the superscript $i$ and focus on an individual real-analytic eigenfunction branch $u_{s}$ with eigenvalue $\nu_{s}$. For $s>0$, we have

$$
\dot{\nu}_{s}=\frac{\dot{\mathcal{A}}_{s}\left(u_{s}\right)}{\mathcal{N}_{s}\left(u_{s}\right)}-\nu_{s} \cdot \frac{\dot{\mathcal{N}}_{s}\left(u_{s}\right)}{\mathcal{N}_{s}\left(u_{s}\right)}
$$

where $\cdot$ indicates differentiation with respect to $s$. A computation gives that for each $w$

and

$$
\dot{\mathcal{A}}_{s}(w)=2 s \cdot \mu \int_{0}^{\infty} x^{2} \cdot g^{\prime}\left(s^{2} \cdot x\right) \cdot w(x)^{2} d x
$$

$$
\dot{\mathcal{N}}_{s}(w)=2 s \int_{0}^{\infty} x \cdot f^{\prime}\left(s^{2} \cdot x\right) \cdot w(x)^{2} d x
$$

\footnotetext{
${ }^{13}$ At $s=0$ the domains of $\mathcal{A}_{s}$ and $\mathcal{N}_{s}$ change, and hence analytic perturbation theory can not be applied.
} 
Let $u_{s}$ be a real-analytic eigenfunction branch of $\mathcal{A}_{s}$ with respect to $\mathcal{N}_{s}$ associated to the real-analytic eigenvalue branch $\nu_{s}$. Integration by parts gives

$$
-u_{s}^{\prime \prime}(x)+\mu \cdot x \cdot g\left(s^{2} \cdot x\right) \cdot u_{s}(x)=\nu_{s} \cdot f\left(s^{2} \cdot x\right) \cdot u_{s}(x) .
$$

Let $M$ be the upper bound on $\nu_{s}$ proven above. If $s \leqslant 1$ and $x>x_{0}:=$ $\max \{1, M / \mu\}$, then

$$
\mu \cdot x \cdot g\left(s^{2} \cdot x\right)-\nu_{s} \cdot f\left(s^{2} \cdot x\right) \geqslant \frac{\mu}{2}
$$

and hence $u_{s}^{\prime \prime} u_{s}(x) \geqslant \frac{\mu}{2} \cdot u_{s}^{2}(x)$ for $s \leqslant 1$. It follows that $\left(u_{s}^{2}\right)^{\prime \prime}(x) \geqslant \mu \cdot u_{s}^{2}(x)$ for $x \geqslant x_{0}$. Thus, since $\mathcal{N}\left(u_{s}\right)$ is finite, we find that for $x_{0} \leqslant x \leqslant y$

$$
\frac{u_{s}(y)^{2}}{u_{s}(x)^{2}} \leqslant \frac{\exp (-\sqrt{\mu} \cdot y)}{\exp (-\sqrt{\mu} \cdot x)}
$$

Integrating from $x_{0}$ to $2 x_{0}$, we find a constant $C$ (that depends on $x_{0}$ ) such that, for $y>2 x_{0}$ we have

$$
y^{2} \cdot u(y)^{2} \leqslant C \cdot y^{2} \exp (-\sqrt{\mu} y) \cdot \int_{x^{0}}^{2 x^{0}} \frac{u(x)^{2}}{1+x^{2}} d x .
$$

From this we find constants $C$ such that that

$$
\left|\dot{\mathcal{A}}_{s}\left(u_{s}\right)\right| \leqslant C \cdot s \cdot \mathcal{N}_{s}\left(u_{s}\right) .
$$

A similar argument shows that

$$
\left|\dot{\mathcal{N}}_{s}\left(u_{s}\right)\right| \leqslant C \cdot s \cdot \mathcal{N}_{s}\left(u_{s}\right) .
$$

Therefore, 1111 holds, and via integration we find $a$ so that 1112 holds true.

Continuity of solutions to ordinary differential equations with respect to coefficients applies to (114) with fixed initial conditions $u_{s}^{\prime}(0)=0$ and $u_{s}(0)=1$. In particular, we have a solution $u_{0}$ to

$$
-u_{0}^{\prime \prime}(x)+2 \mu \cdot x \cdot u_{0}(x)=a \cdot u_{0}(x) .
$$

It follows that

$$
\left.v(z):=u_{0}\left((2 \mu)^{-\frac{1}{3}} \cdot z+(2 \mu)^{-1} \cdot a\right)\right)
$$

is a solution to $v^{\prime \prime}(z)=z \cdot v(z)$. Estimate $115 p$ applies to $u_{0}$, and hence it follows from (161) that $v$ is a multiple of $A_{-}$. The function $u$ satisfies the Neumann condition $u^{\prime}(0)=0$ and hence $v^{\prime}\left(-(2 \mu)^{-\frac{2}{3}} \cdot a\right)=0$ as desired.

Proof of Proposition A.1. If $v_{t}$ is a real-analytic eigenfunction branch of of $a_{t}^{\ell}$ associated to the eigenvalue branch $\lambda_{t}$, then $\bar{v}_{s^{3}}$ is a real-analytic eigenfunction branch of $\mathcal{A}_{s}$ with eigenvalue branch $\nu_{s}=s^{-2}\left(\lambda_{s^{3}}-\mu\right)$. Lemma A.2 implies that

$$
\lambda_{t}=\mu+a \cdot t^{\frac{2}{3}}+O\left(t^{\frac{4}{3}}\right) .
$$

By differentiating $\lambda_{s^{3}}=\mu+s^{2} \cdot \nu_{s}$, we find that

$$
3 \dot{\lambda}_{s^{3}}=\dot{\nu}_{s}+2 \cdot \nu_{s} \cdot s^{-1} \text {. }
$$

By Lemma A.2 both $\dot{\nu}_{s}$ and $\nu_{s}$ are bounded. Therefore, $\dot{\lambda}_{s^{3}}=O\left(s^{-1}\right)$ and hence $\dot{\lambda}_{t}=O\left(t^{-\frac{1}{3}}\right)$. 


\section{Appendix B. The off-Diagonal estimates}

Let $\left(E_{t}, u_{t}\right)$ be a real-analytic eigenbranch of $q_{t}$ such that $\lim _{t \rightarrow 0} E_{t}=E_{0}=$ $(\pi \cdot k)^{2}$ for some positive integer $k$. For a fixed constant $C>0$, let

$$
I=\left[E_{0}-C, E_{0}+C\right] \text {. }
$$

As in $\$ 7.2$, let $w_{t}$ denote the orthogonal projection of $u_{t}$ onto the sum of the eigenspaces of $a_{t}$ whose eigenvalues lie in $I$.

The purpose of this appendix is to prove the following fact that is crucially used in the proof of Proposition 8.8. We recall that $b_{t}$ is the quadratic form defined in (27).

Proposition B.1. Let $\eta>0$. There exists $\kappa>0, \delta>0$, and $t_{0}>0$ such that, if $t<t_{0}$ and if $\psi^{0}$ is an eigenfunction of $a_{t}^{0}$ with eigenvalue $\lambda^{0}$ satisfying

$$
\left|\lambda^{0}-E_{t}\right| \leqslant \eta \cdot t^{\frac{5}{3}}
$$

then

$$
\left|b_{t}\left(u_{t}, \psi^{0} \otimes 1\right)\right| \geqslant \kappa \cdot t^{\frac{2}{3}} \cdot\left(\left\|w_{t}^{k}\right\|-t^{\delta} \cdot\left\|u_{t}\right\|\right) \cdot\left\|\psi^{0}\right\| .
$$

Remark B.2. The condition on $\lambda^{0}$ is only used to ensure that, when $t$ tends to 0 , $\lambda^{0}$ tends to $k^{2} \pi^{2}$.

Proof. Proposition 6.2 says that the quadratic form $b_{t}$ is controlled by $\widetilde{a}_{t}$ : there exists a constant $C$ such that for $u, v \in \operatorname{dom}\left(a_{t}\right)$

$$
\left|b_{t}(u, v)\right| \leqslant C \cdot \widetilde{a}_{t}(u)^{\frac{1}{2}} \cdot \widetilde{a}_{t}(v)^{\frac{1}{2}} .
$$

Thus, lemma 7.5 and the fact that $\widetilde{a}_{t}\left(\psi^{0}\right)=O\left(\left\|\psi_{0}\right\|^{2}\right)$ imply that

$$
b_{t}\left(u_{t}-w_{t}, \psi^{0} \otimes 1\right)=O(t) \cdot\left\|u_{t}\right\| \cdot\left\|\psi^{0}\right\|,
$$

and hence it suffices to bound $b_{t}\left(w_{t}, \psi^{0}\right)$ from below.

Observe also that lemma 7.5 also implies that $\left\|u_{t}\right\| \sim\left\|w_{t}\right\|$ in the limit $t \rightarrow 0$ so that we can freely replace $\left\|u_{t}\right\|$ by $\left\|w_{t}\right\|$ and vice-versa in each (multiplicative) estimate.

By the discussion $\$ 6.2$ and $\$ 7.2$, for each $t$ we can uniquely write

$$
w_{t}(x, y)=\sum_{\ell \leqslant k} \sum_{\lambda \in \operatorname{spec}\left(a_{t}^{\ell}\right) \cap I_{t}} \psi_{\lambda}^{\ell}(y) \cdot e_{\ell}(x)
$$

where each $\psi_{\lambda}^{\ell}(y)$ is an eigenfunction of $a_{t}^{\ell}$ with eigenvalue $\lambda \in I_{t}$. Set

$$
v_{t}^{\ell}(y)=\sum_{\lambda \in \operatorname{spec}\left(a_{t}^{\ell}\right) \cap I_{t}} \psi_{\lambda}^{\ell}(y) .
$$

and

By linearity

$$
w_{t}^{\ell}(x, y)=v_{t}^{\ell}(y) \cdot e_{\ell}(x)
$$

$$
b_{t}\left(w_{t}, \psi^{0} \otimes 1\right)=\sum_{\ell \leqslant k} b_{t}\left(w_{t}^{\ell}, \psi^{0} \otimes 1\right) .
$$

From (27) we have

$$
b_{t}\left(w_{t}^{\ell}, \psi^{0} \otimes 1\right)=\int_{1}^{\underline{\alpha}} \int_{0}^{1} \widetilde{\nabla}_{t} w_{t}^{\ell} \cdot\left(\begin{array}{cc}
0 & x \cdot p(y) \\
x \cdot p(y) & 0
\end{array}\right) \cdot\left(\widetilde{\nabla}_{t} \psi^{0}(y)\right)^{*} d x d y .
$$


where $\widetilde{\nabla}_{t} f=\left[\partial_{x} f, t \partial_{y} f\right]$ and $p(y)$ is defined in 25$)$. Since $\partial_{x} \psi(y) \equiv 0$, and $e_{\ell}(x)=$ $2^{-\frac{1}{2}} \cos (\ell \pi x)$, we find that

$b_{t}\left(w_{t}^{\ell}, \psi^{0} \otimes 1\right)=\left(-2^{-\frac{1}{2}} \ell \pi \cdot \int_{0}^{1} x \cdot \sin (\ell \pi x) d x\right) \cdot\left(\int_{1}^{\underline{\alpha}} p(y) \cdot v_{t}^{\ell}(y) \cdot\left(t \cdot\left(\psi^{0}\right)^{\prime}(y)\right) d y\right)$

If $\ell=0$, then $\sin (\ell \pi x) \equiv 0$, and so $b_{t}\left(w_{t}^{\ell}, \psi^{0} \otimes 1\right)=0$. For $0<\ell<k$, apply Lemma B.3 below to find that

$$
\left|b_{t}\left(w_{t}^{\ell}, \psi^{0} \otimes 1\right)\right|=O_{\ell}(t) \cdot\left\|v_{t}^{\ell}\right\| \cdot\left\|\psi^{0}\right\|
$$

Since $w_{t}^{\ell}$ and $w_{t}^{\ell^{\prime}}$ are orthogonal if $\ell \neq \ell^{\prime}$, we have

$$
\sum_{\ell=1}^{k-1}\left\|v_{t}^{\ell}\right\|^{2}=2^{-\frac{1}{2}} \sum_{\ell=1}^{k-1}\left\|w_{t}^{\ell}\right\|^{2} \leqslant\left\|w_{t}\right\|^{2}
$$

Thus, by summing 120 over $\ell \in\{0, \ldots, k-1\}$, we obtain

$$
\begin{aligned}
\left|\sum_{\ell=0}^{k-1} b_{t}\left(w_{t}^{\ell}, \psi^{0} \otimes 1\right)\right| & \leqslant O(t) \cdot\left(\sum_{1}^{k-1}\left\|v_{t}^{\ell}\right\|\right) \cdot\left\|\psi_{t}^{0}\right\| \\
& \leqslant O(t)\left(\sum_{1}^{k-1}\left\|v_{t}^{\ell}\right\|^{2}\right)^{\frac{1}{2}} \cdot\left\|\psi_{t}^{0}\right\| \\
& \leqslant O(t) \cdot\left\|w_{t}\right\| \cdot\left\|\psi_{t}^{0}\right\|
\end{aligned}
$$

For $\ell=k$, we have

$$
k \pi \int_{0}^{1} x \sin (k \pi x) d x=(-1)^{k} \neq 0
$$

Thus, from Lemma B.4 and Lemma 5.1, there exists $\kappa^{\prime}>0$ so that

$$
\left|b_{t}\left(w_{t}^{k}, \psi^{0} \otimes 1\right)\right| \geqslant \kappa^{\prime} \cdot t^{\frac{2}{3}} \cdot\left(\left\|w_{t}^{k}\right\|-t^{\delta}\left\|u_{t}\right\|\right) \cdot\left\|\psi^{0}\right\|
$$

for some $\kappa^{\prime}>0$. The latter estimate, combined with 119, 121 , and the triangle inequality, yield the claim.

Lemma B.3. For each smooth function $g:[1, \underline{\alpha}] \rightarrow \mathbb{R}$, there exists $C>0$ and $t_{0}>0$ such that if $t \leqslant t_{0}$ and $0<\ell<k$, then

$$
\left|\int_{1}^{\underline{\alpha}} g(y) \cdot v_{t}^{\ell}(y) \cdot\left(t \cdot\left(\psi^{0}\right)^{\prime}(y)\right) d y\right| \leqslant C \cdot t \cdot\left\|u_{t}\right\| \cdot\left\|\psi^{0}\right\|
$$

Lemma B.4. For each smooth function $g:[1, \underline{\alpha}] \rightarrow \mathbb{R}$ with $g(1) \neq 0$, there exists $\kappa, \delta, t_{0}>0$ such that, for each $t<t_{0}$

$$
\left|\int_{1}^{\underline{\alpha}} g(y) \cdot v_{t}^{k}(y) \cdot\left(t \cdot\left(\psi^{0}\right)^{\prime}(y)\right) d y\right| \geqslant \kappa \cdot t^{\frac{2}{3}} \cdot\left(\left\|w_{t}^{k}\right\|-t^{\delta}\left\|u_{t}\right\|\right) \cdot\left\|\psi^{0}\right\| .
$$

The remainder of this appendix is devoted to proving the preceding lemmas. 
B.1. The proof of Lemma B.3. Define $r_{t}^{\ell}:[1, \infty[\rightarrow \mathbb{R}$ by

$$
r_{t}^{\ell}(y)=t^{2} \cdot\left(v_{t}^{\ell}\right)^{\prime \prime}(y)+\left(\frac{E_{t}}{y^{2}}-(\ell \cdot \pi)^{2}\right) \cdot v_{t}^{\ell}(y)
$$

Lemma B.5. There exists $t_{0}>0$ and $C$ so that if $t<t_{0}$, then for each $\ell \in \mathbb{N}$

$$
\int_{1}^{\infty}\left|r_{t}^{\ell}(y)\right|^{2} d y \leqslant C \cdot t^{2} \cdot\left\|u_{t}\right\|^{2}
$$

Proof. Multiply both sides of 123 by a smooth function with compact support $\phi^{\ell}$ and integrate over $y \in[1, \infty[$, then integrate by parts to obtain

$$
\int_{1}^{\infty} r_{t}^{\ell}(y) \phi^{\ell}(y) d y=-a_{t}^{\ell}\left(v_{t}^{\ell}, \phi\right)+E_{t} \cdot\left\langle v_{t}^{\ell}, \phi\right\rangle .
$$

Observe that $a_{t}^{\ell}\left(v_{t}^{\ell}, \phi^{\ell}\right)-E_{t} \cdot\left\langle v_{t}^{\ell}, \phi\right\rangle=a_{t}\left(w_{t}, \phi^{\ell} \otimes e_{\ell}\right)-E_{t}\left\langle w_{t}, \phi^{\ell} \otimes e_{\ell}\right\rangle$ so that by applying Lemma 7.8 to the test function $\phi^{\ell} \otimes e_{\ell}$, there exists $t_{0}>0$ and $C^{\prime}$ such that for $t<t_{0}$, we have

$$
\left|\int_{1}^{\infty} r_{t}^{\ell}(y) \phi^{\ell}(y) d y\right| \leqslant C^{\prime} \cdot t \cdot\left\|u_{t}\right\| \cdot\left\|\phi^{\ell}\right\| .
$$

Recalling that the $L^{2}$-norm on the right hand side has the weight $y^{-2}$, this implies that

$$
\int_{1}^{\infty} y^{2}\left|r_{t}^{\ell}(y)\right|^{2} d y \leqslant\left(C^{\prime} \cdot t \cdot\left\|u_{t}\right\|\right)^{2}
$$

The claim follows since $y^{2} \geqslant 1$ on the interval over which we integrate.

The strategy of the proof of Lemma B.3 is as follows. By 123 , the function $v_{t}^{\ell}$ is a solution to the inhomogeneous equation

$$
t^{2} \cdot v^{\prime \prime}+f_{\mu}^{\ell} \cdot v=r
$$

where $\mu=E_{t}$ and

$$
f_{\mu}^{\ell}(y):=\frac{\mu}{y^{2}}-(\ell \cdot \pi)^{2} .
$$

The function $\psi_{t}^{0}$ is a solution to the homogeneous equation

$$
v^{\prime \prime}+t^{-2} \cdot f_{\mu}^{\ell} \cdot v=0
$$

where $\mu=\lambda^{0}$. Our choice of $\beta$ in 73 implies that $f_{\mu}^{\ell}$ is bounded below by a constant $\delta_{1}>0$ for all small $t, \ell<k$, and $\mu \in I$. Hence we can use WKB type estimates to find a basis $v_{ \pm}$of solutions to the homogeneous equation 126 . We will then use 'variation of parameters' to express each solution to 124 in terms of this basis, and we use Lemma B.5 to provide control of the inhomogeneous term $r$. Finally, we will estimate the integral in 122 using a Riemann-Lebesgue type estimate.

Proof of Lemma B.3. For $\ell<k$ and $\mu \in I$, we have $f_{\mu}^{\ell} \geqslant \delta_{1}>0$, and hence we can apply Theorem 6.2.1 in Olver, to obtain a basis $\left(v_{\mu,+}^{\ell}, v_{\mu,-}^{\ell}\right)$ of solutions to the homogeneous equation 126 that satisfy

$$
v_{\mu, \pm}^{\ell}(y)=\left|f_{\mu}^{\ell}(y)\right|^{-\frac{1}{4}} \exp \left( \pm \frac{i}{t} \int_{1}^{y}\left|f_{\mu}^{\ell}(z)\right|^{\frac{1}{2}} d z\right)(1+\varepsilon(y))
$$


and

$$
t \cdot\left(v_{\mu, \pm}^{\ell}\right)^{\prime}(y)= \pm i \cdot\left|f_{\mu}^{\ell}(y)\right|^{\frac{1}{4}} \cdot \exp \left( \pm \frac{i}{t} \int_{1}^{y}\left|f_{\mu}^{\ell}(z)\right|^{\frac{1}{2}} d z\right)(1+\bar{\varepsilon}(y))
$$

where, for $\mu \in I$ and $\ell<k$, the smooth functions $\varepsilon$ and $\bar{\varepsilon}$ have $C^{0}$ norm that is uniformly $O(t)$.

Observe that $v_{\mu, \pm}^{\ell}$ have $L^{2}([1, \beta])$ norms that are uniformly bounded above and away from 0 . Moreover, since $v_{\mu,+}^{\ell}$ and $v_{\mu,-}^{\ell}$ are highly oscillatory for small $t$, an integration by parts argument shows that the $L^{2}([1, \beta])$ inner product $\left\langle v_{\mu,+}^{\ell}, v_{\mu,-}^{\ell}\right\rangle$ is $O(t)$. It follows that there exists $m>0$ such that if $\left(a_{+}, a_{-}\right) \in \mathbb{C}$, then

$$
m \cdot\left(\left|a_{+}\right|^{2}+\left|a_{-}\right|^{2}\right)^{\frac{1}{2}} \leqslant\left\|a_{+} \cdot v_{\mu,+}^{\ell}+a_{-} \cdot v_{\mu,-}^{\ell}\right\| \leqslant m^{-1} \cdot\left(\left|a_{+}\right|^{2}+\left|a_{-}\right|^{2}\right)^{\frac{1}{2}} .
$$

for all sufficiently small $t$. Here $\|\cdot\|$ denotes the $L^{2}([1, \beta])$ norm.

By the method of 'variation of constants', each solution to

$$
v^{\prime \prime}+t^{-2} \cdot f_{\mu}^{\ell} \cdot v=t^{-2} \cdot r
$$

is of the form

$$
v=\left(a_{+}+h_{\mu,+}^{\ell, r}\right) \cdot v_{\mu,+}^{\ell}+\left(a_{-}+h_{\mu,-}^{\ell, r}\right) \cdot v_{\mu,-}^{\ell}
$$

where $\left(a_{+}, a_{-}\right) \in \mathbb{C}^{2}$,

$$
h_{\mu, \pm}^{\ell, r}(y)= \pm t^{-2} \cdot \mathcal{W}^{-1} \int_{1}^{y} r(z) \cdot v_{\mu, \mp}^{\ell}(z) d z
$$

and $\mathcal{W}=v_{\mu,+}^{\prime} \cdot v_{\mu,-}-v_{\mu,-}^{\prime} \cdot v_{\mu,+}$ is the Wronskian.

In particular, for each $\ell$ and each $t$, there exists $\left(a_{t,+}^{\ell}, a_{t,-}^{\ell}\right) \in \mathbb{C}^{2}$ so that the function $v_{t}^{\ell}$ of (118) satisfies

$$
v_{E_{t}}^{\ell}=\left(a_{t,+}^{\ell}+h_{E_{t},+}^{\ell, r_{t}^{\ell}}\right) \cdot v_{E_{t},+}^{\ell}+\left(a_{t,-}^{\ell}+h_{E_{t,-}}^{\ell, r_{t}^{\ell}}\right) \cdot v_{E_{t},-}^{\ell}
$$

The eigenfunction $\psi^{0}$ of $a_{t}^{0}$ satisfies 130 with $r=0$, and hence there exists $\left(c_{+}, c_{-}\right) \in \mathbb{C}^{2}$ so that

$$
\psi^{0}=c_{+} \cdot v_{\lambda^{0},+}^{0}+c_{-} \cdot v_{\lambda^{0},-}^{0} .
$$

The integral in 122 is equal to

$$
\int_{1}^{\underline{\alpha}} g \cdot\left(\sum_{ \pm}\left(a_{t, \pm}^{\ell}+h_{E_{t}, \pm}^{\ell, r_{t}^{\ell}}\right) \cdot v_{E_{t} \pm}^{\ell}\right) \cdot\left(\sum_{ \pm} c_{ \pm} \cdot\left(t \cdot v_{\lambda^{0}, \pm}^{0}\right)^{\prime}\right) d y
$$

By expanding the product of sums, one obtains a sum of $2^{3}$ integrals. By substituting the expressions (128) and (127), integration by parts, and applying standard estimates, we find that each integral is $O(t)$.

For example, consider the terms of the form

$$
a_{t, \pm}^{\ell} \cdot c_{ \pm} \int_{1}^{\underline{\alpha}} g \cdot\left(\frac{f_{\lambda^{0}}^{0}}{f_{E_{t}}^{\ell}}\right)^{\frac{1}{4}} \exp \left(\frac{i}{t} \int_{1}^{y} \pm\left(f_{E_{t}}^{\ell}\right)^{\frac{1}{2}} \mp\left(f_{\lambda_{0}}^{0}\right)^{\frac{1}{2}}\right) \cdot\left(1+\varepsilon^{*}\right) d y .
$$

Since $\ell>0$, an elementary computation shows that there exists $\delta>0$ so that if $z \in[1, \beta]$ and $t$ is sufficiently small, then

$$
\delta \leqslant\left|\left(f_{E_{t}}^{\ell}(z)\right)^{\frac{1}{2}} \pm\left(f_{\lambda^{0}}^{0}(z)\right)^{\frac{1}{2}}\right|
$$


Thus, we may integrate by parts to find a constant $C$ so that the integral in 132 is at most $C \cdot t \cdot\|g\|_{C^{1}}$. It follows that all the terms of this form are bounded above by

$$
C^{\prime} \cdot t \cdot\|g\|_{C^{1}} \cdot\left\|\psi^{0}\right\| \cdot\left(\sum_{ \pm}\left|a_{t, \pm}^{\ell}\right|\right) .
$$

We also have terms of the form

$$
c_{ \pm} \int_{1}^{\underline{\alpha}} g \cdot h_{E_{t}, \pm}^{\ell, r_{t}^{\ell}} \cdot\left(\frac{f_{\lambda^{0}}^{0}}{f_{E_{t}}^{\ell}}\right)^{\frac{1}{4}} \exp \left(\frac{i}{t} \int_{1}^{y} \pm\left(f_{\mu}^{\ell}\right)^{\frac{1}{2}} \mp\left(f_{\mu}^{0}\right)^{\frac{1}{2}}\right) \cdot\left(1+\varepsilon^{*}\right) d y .
$$

We integrate by parts as above, but this time we need to also bound $h_{ \pm}=h_{E_{t}, \pm}^{\ell, r_{t}^{\ell}}$ and its derivative.

From (127) and (128) we find that there exists $t_{1}$ so that if $t<t_{1}$, then $|t \cdot \mathcal{W}| \geqslant 1$. From (127) and (125) we find that for each $\ell$

$$
\sup _{y \in[1, \beta]}\left|v_{E_{t}, \mp}^{\ell}(y)\right| \leqslant \frac{2}{\sqrt{\delta_{1}}}
$$

for all sufficiently small $t$. Hence, using the Cauchy-Schwarz inequality and Lemma B.5 we have, for all $y \in[1, \beta]$,

$$
\begin{aligned}
\left|h_{ \pm}(y)\right| & \leqslant \frac{1}{t} \cdot\left|\int_{1}^{y} r(y) \cdot v_{E_{t}, \mp}^{\ell}(y) d y\right| \\
& \leqslant \frac{1}{t} \cdot\left(\int_{1}^{y} r(y)^{2} d y\right)^{\frac{1}{2}} \cdot\left(\int_{1}^{y} v_{E_{t}, \mp}^{\ell}(y)^{2} d y\right)^{\frac{1}{2}} \\
& \leqslant \frac{1}{t} \cdot C \cdot t \cdot\left\|u_{t}\right\| \cdot \sqrt{\beta-1} \cdot 2 \cdot \delta_{1}^{-\frac{1}{2}}
\end{aligned}
$$

for all sufficiently small $t$. For the derivative of $h_{\mu, \pm}^{\ell, r}$, we have

$$
\left|h_{ \pm}^{\prime}(y)\right| \leqslant \frac{3}{t} \cdot|r(y)| \cdot 2 \delta^{-\frac{1}{2}}
$$

Applying Cauchy-Schwarz and Lemma B.5 gives

$$
\begin{aligned}
\int_{1}^{\underline{\alpha}}\left|h_{ \pm}^{\prime}(y)\right| d y & \leqslant \frac{6 \cdot \delta^{-\frac{1}{2}}}{t} \sqrt{\beta-1} \cdot\left(\int_{1}^{\beta}|r(y)|^{2} d y\right)^{\frac{1}{2}} \\
& \leqslant \frac{2 \cdot \delta^{-\frac{1}{2}}}{t} \sqrt{\beta-1} \cdot C \cdot t \cdot\left\|u_{t}\right\| .
\end{aligned}
$$

Finally, we apply integration by parts to 135 . The resulting terms that do not contain $h_{ \pm}^{\prime}$ have uniformly bounded $C^{0}$ norm. The term that contains $h_{ \pm}^{\prime}$ can be bounded using (137). It follows that all the term of this form are bounded by

$$
C \cdot t \cdot\left\|u_{t}\right\| \cdot\left\|\psi^{0}\right\| \text {. }
$$

The final step consists in bounding $\sum\left|a_{ \pm}\right|$by $\left\|v_{t}^{\ell}\right\|$ to control the terms of eq. 134.

Using (129) and (131) we have

$$
m\left(\left|a_{+}\right|^{2}+\left|a_{-}\right|^{2}\right)^{\frac{1}{2}} \leqslant\left\|v_{t}^{\ell}\right\|+(\beta-1) \cdot \frac{2}{\sqrt{\delta_{1}}} \sup _{[1, \beta]}\left\{\left|h_{+}(y)\right|+\left|h_{-}(y)\right|\right\}
$$


By orthogonality we have $\left\|v_{t}^{\ell}\right\| \leqslant\left\|u_{t}\right\|$ and, using the bound on $\left|h_{ \pm}(y)\right|$ we finally obtain

$$
\left(\left|a_{+}\right|^{2}+\left|a_{-}\right|^{2}\right)^{\frac{1}{2}} \leqslant C \cdot\left\|u_{t}\right\| \cdot
$$

This finishes the proof.

B.2. The proof of Lemma B.4. As in the previous subsection, the function $v_{t}^{k}$ is a solution to the inhomogeneous equation (124) with $\mu=E_{t}$ and $r$ defined by (123). However, for $\ell=k$, the function

$$
f_{t}^{k}(y)=\frac{E_{t}}{y^{2}}-k^{2} \pi^{2}
$$

is negative for large $y$. In fact, since $E_{t}$ decreases to $(\pi k)^{2}$, the function $f_{t}^{k}$ changes sign nearer and nearer to $y=1$. Since the solution $v_{t}^{k}$ belongs to $L^{2}\left(\mathbb{R}, y^{-2} d x d y\right)$, we expect it to decay exponentially as soon as $y$ moves away from 1. For $y$ near 1 , we will approximate $v_{t}^{\ell}$ using Airy functions. In this subsection we will make these approximations precise and use them to give a proof of Lemma B.4

B.2.1. Normalization of $\psi^{0}$. By Lemma 6.3, $\psi^{0}$ is a constant multiple of $\psi$ defined in (37). Because both sides of the estimate in Lemma B.4 are homogeneous functions of degree 1 in $\psi^{0}$, it suffices to assume that $\psi^{0}=\psi$.

Let $|f|_{0}$ denote the supremum norm of $f$ over $[1, \beta]$.

Lemma B.6. There exists $t_{0}>0$ such that if $t<t_{0}$ and $\lambda^{0} \in I$, then

$$
\frac{1}{2} \leqslant|\psi|_{0} \leqslant 2 \sqrt{\beta},
$$

$$
\frac{\sqrt{\ln (\beta)}}{2} \leqslant\|\psi\| \leqslant \sqrt{\ln (\beta)}
$$

and

$$
\left|t \cdot \psi^{\prime}\right|_{0} \leqslant 2 \sqrt{\sup (I)} .
$$

Proof. We have

$$
\psi(y)=\omega^{+}(r, y)-(2 r)^{-1} \cdot \omega^{-}(r, y)
$$

where

$$
\begin{aligned}
& \omega^{+}(r, y)=y^{\frac{1}{2}} \cdot \cos (r \cdot \ln (y)), \\
& \omega^{-}(r, y)=y^{\frac{1}{2}} \cdot \sin (r \cdot \ln (y)),
\end{aligned}
$$

and $\lambda^{0}=t^{2} \cdot\left(1 / 4+r^{2}\right)$. In particular, for sufficiently small $t$

$$
\frac{\sqrt{\inf (I)}}{2 t} \leqslant r \leqslant \frac{\sqrt{\sup (I)}}{t} .
$$

Thus, since $\left|\omega^{ \pm}\right|_{0} \leqslant \sqrt{\beta}$ and $\left|\omega^{+}\right|_{0} \geqslant 1$, the triangle inequality applied to 142 implies that 139 holds for sufficiently small $t$.

$$
\begin{aligned}
\int_{1}^{\beta}\left|\omega_{+}(y)\right|^{2} y^{-2} d y & =\int_{1}^{\beta} \mid \cos \left(\left.r \ln (y)\right|^{2} \frac{d y}{y}\right. \\
& =\int_{0}^{\ln \beta}|\cos (r z)|^{2} d z \\
& =\frac{1}{2} \ln \beta+O\left(\frac{1}{r}\right) .
\end{aligned}
$$


The same estimate applies for $\omega_{-}$. Hence the triangle inequality and 143 imply that 140 holds for sufficiently small $t$.

The bound on $t \cdot \psi^{\prime}$ is proven in a similar fashion using the fact that

$$
\psi^{\prime}(y)=-\left(r+\frac{1}{4 r}\right) \cdot y^{-1} \cdot \omega^{-}(r, y)
$$

together with 143 and the fact that $r$ is of order $t^{-1}$.

B.2.2. Localization near $y=1$. The following proposition provides a quantitative description of the concentration of solutions to $t^{2} \cdot v^{\prime \prime}+f_{t}^{k} \cdot v=r$ near $y=1$.

Proposition B.7. Let $k \in \mathbb{Z}^{+}$. For each $\left.\alpha \in\right] 0, \frac{2}{3}\left[\right.$, there exists $t_{0}>0$ and $C$, such that if $v$ is a solution to $t^{2} \cdot v^{\prime \prime}+f_{t}^{k} \cdot v=r$ and $t<t_{0}$,

$$
\int_{1+2 t^{\alpha}}^{\infty}|v(y)|^{2} d y \leqslant C \cdot\left(t^{-2 \alpha} \cdot \int_{1}^{\infty}|r|^{2}+\exp \left(-t^{\frac{3 \alpha-2}{2}}\right) \cdot \int_{1+t^{\alpha}}^{\infty} v^{2}(y) y^{-2} d y\right) .
$$

Proof. By Proposition 8.3 and Lemma A.1, there exists $C$ so that if $t$ is sufficiently small, then

$$
E_{t} \leqslant(k \pi)^{2}+C \cdot t^{\frac{2}{3}}
$$

Hence, for $y \geqslant 1+t^{\alpha}$, one finds that

$$
(k \pi)^{2}-\frac{E_{t}}{y^{2}} \geqslant(k \pi)^{2} \cdot\left(\frac{2 \cdot t^{\alpha}+t^{2 \alpha}}{1+2 t^{\alpha}+t^{2 \alpha}}\right)-C \cdot t^{\frac{2}{3}} .
$$

Thus, since $\alpha<2 / 3$, there exists $t_{1}>0$ such that if $t \leqslant t_{1}$ then for all $y \geqslant 1+t^{\alpha}$ we have

$$
(k \pi)^{2}-\frac{E_{t}}{y^{2}} \geqslant(k \pi)^{2} \cdot t^{\alpha}
$$

For each smooth function $\varphi$ with support in $] 1+t^{\alpha}, \infty[$, define

$$
L_{t}(\varphi)=-t^{2} \cdot \varphi^{\prime \prime}+\left((k \pi)^{2}-\frac{E_{t}}{y^{2}}\right) \cdot \varphi
$$

Extend $L_{t}$ to a self-adjoint operator on $L^{2}\left(\left[1+t^{\alpha}, \infty[, d y)\right.\right.$. It follows from 148) that the spectrum of $L_{t}$ lies in $\left[(k \pi)^{2} \cdot t^{\alpha}, \infty\left[\right.\right.$. Hence $L_{t}$ is invertible and the operator norm of $\left\|L_{t}^{-1}\right\|$ is bounded above by $\frac{t^{-\alpha}}{k^{2} \pi^{2}}$. Therefore

$$
\int_{1+t^{\alpha}}^{\infty}\left|L_{t}^{-1}(r)\right|^{2} \frac{d y}{y^{2}} \leqslant \int_{1+t^{\alpha}}^{\infty}\left|L_{t}^{-1}(r)\right|^{2} d y \leqslant \frac{t^{-2 \alpha}}{(k \pi)^{4}} \int_{1+t^{\alpha}}^{\infty}|r(y)|^{2} d y .
$$

The function $L_{t}^{-1}(r)$ is a solution to 124 on $\left[1+t^{\alpha}, \infty\right)$, and hence $w:=$ $v-L_{t}^{-1}(r)$ is a solution to the homogeneous equation 126 . It follows from 148 that

$$
\left(w^{2}\right)^{\prime \prime}(y) \geqslant t^{\alpha-2} \cdot w^{2}(y) .
$$

if $y \in\left[1+t^{\alpha}, \infty\right.$ [. In particular, $w^{2}$ is convex, moreover $w^{2}$ is non-negative and in $L^{2}\left(\left[1+t^{\alpha}, \infty\right), y^{-2} d y\right)$ since $v \in L^{2}\left(\left[1+t^{\alpha}, \infty\right), y^{-2} d y\right)$ and $L_{t}^{-1} r \in L^{2}([1+$ $\left.\left.t^{\alpha}, \infty\right), d y\right) \subset L^{2}\left(\left[1+t^{\alpha}, \infty\right), y^{-2} d y\right)$. This implies that $\lim _{y \rightarrow \infty} w^{2}(y)=0$. Indeed, since $w^{2}$ is convex, $\left(w^{2}\right)^{\prime}$ has a limit $m$ in $\mathbf{R} \cup\{+\infty\}$. If this limit is positive then it implies that $w^{2}(y) \geqslant \frac{m}{2} y$ for large $y$ and this contradicts the fact that $w^{2}(y) y^{-2}$ is integrable. In particular, $\left(w^{2}\right)^{\prime}$ is bounded so that by integrating 150 we find that $w^{2} \in L^{1}\left(\left[1+t^{\alpha}, \infty\right), d y\right)$. The argument also shows that $\left(w^{2}\right)^{\prime}$ is non positive 
for large $y$ so that $w^{2}$ has a limit when $y \rightarrow \infty$. Since $w^{2}$ is integrable, this limit is 0.

For each $y \in\left[1+t^{\alpha}, \infty\right)$ the function $e_{y}$, that is defined by

$$
e_{y}(z)=w^{2}(y) \cdot \exp \left(-t^{\frac{\alpha-2}{2}} \cdot(z-y)\right)
$$

satisfies $e_{y}^{\prime \prime}(z)=t^{\alpha-2} \cdot e_{y}(z)$ with $e_{y}(y)=w^{2}(y)$ and $\lim _{z \rightarrow \infty} e_{y}(z)=0$. Therefore, by comparison with (150), and using the maximum principle, we find that if $z \geqslant y$, then $w^{2}(z) \leqslant e_{z}(y)$. Applying this to $z=y+t^{\alpha}$, we find that for each $y \geqslant 1+t^{\alpha}$

$$
w^{2}\left(y+t^{\alpha}\right) \leqslant \exp \left(-t^{\frac{3 \alpha-2}{2}}\right) w^{2}(y)
$$

By integration we obtain

$$
\int_{1+2 t^{\alpha}}^{\infty} w^{2}(y) d y \leqslant \exp \left(-t^{\frac{3 \alpha-2}{2}}\right) \cdot \int_{1+t^{\alpha}}^{\infty} w^{2}(y) d y
$$

This implies

$$
\int_{1+2 t^{\alpha}}^{\infty} w^{2}(y) d y \leqslant \frac{\exp \left(-t^{\frac{3 \alpha-2}{2}}\right)}{1-\exp \left(-t^{\frac{3 \alpha-2}{2}}\right)} \cdot \int_{1+t^{\alpha}}^{1+2 t^{\alpha}} w^{2}(y) d y
$$

It follows that for $t$ small enough we have

$$
\begin{aligned}
\int_{1+2 t^{\alpha}}^{\infty} w^{2}(y) d y & \leqslant \exp \left(-t^{\frac{3 \alpha-2}{2}}\right) \int_{1+t^{\alpha}}^{1+2 t^{\alpha}} w^{2}(y) d y \\
& \leqslant \frac{1}{2} \exp \left(-t^{\frac{3 \alpha-2}{2}}\right) \int_{1+t^{\alpha}}^{1+2 t^{\alpha}} w^{2}(y) y^{-2} d y \\
& \leqslant \frac{1}{2} \exp \left(-t^{\frac{3 \alpha-2}{2}}\right) \int_{1+t^{\alpha}}^{\infty} w^{2}(y) y^{-2} d y
\end{aligned}
$$

We now use 149 and the triangle inequality to obtain

$$
\begin{aligned}
\|v\|_{\left[1+2 t^{\alpha},+\infty\right)} \leqslant & \|w\|_{\left[1+2 t^{\alpha},+\infty\right)}+\left\|L_{t}^{-1}(r)\right\|_{\left[1+2 t^{\alpha},+\infty\right)} \\
\leqslant & \exp \left(-t^{\frac{3 \alpha-2}{2}} / 2\right)\|w\|_{\left[1+t^{\alpha},+\infty\right), y^{-2}}+C t^{-\alpha}\|r\|_{[1 ;+\infty)} \\
& \leqslant \exp \left(-t^{\frac{3 \alpha-2}{2}} / 2\right)\left(\|v\|_{\left[1+t^{\alpha},+\infty\right), y^{-2}}+\left\|L_{t}^{-1}(r)\right\|_{\left[1+t^{\alpha},+\infty\right), y^{-2}}\right) \\
& +C t^{-\alpha}\|r\|_{[1,+\infty)}
\end{aligned}
$$

The claim follows.

Corollary B.8. For each $\alpha \in] 0, \frac{2}{3}\left[\right.$, there exists $C$ and $t_{0}$ such that, for each $t<t_{0}$,

$$
\int_{1+2 t^{\alpha}}^{\infty}\left|v_{t}^{k}(y)\right|^{2} \frac{d y}{y^{2}} \leqslant C \cdot t^{2-2 \alpha}\left\|u_{t}\right\|^{2} .
$$

Proof. Using orthogonality, we have that $\left\|v_{t}^{k}\right\|_{L^{2}\left(\frac{d y}{y^{2}}\right)} \leqslant\left\|w_{t}\right\| \sim\left\|u_{t}\right\|$. Since $y^{-2} \leqslant 1$ on the interval $\left[1+t^{\alpha}, \infty\right)$, the integral with $v_{t}^{k}$ on the right-hand side is bounded by $C\left\|u_{t}\right\|^{2}$. The integral with $r$ is controlled via Lemma B.5.

Corollary B.9. There exist $C$ and $t_{0}>0$ so that if $t<t_{0}$

$$
\left|\int_{1+2 t^{\alpha}}^{\underline{\alpha}} g(y) \cdot v_{t}^{k}(y) \cdot(t \psi)^{\prime}(y) d y\right| \leqslant C \cdot t^{1-\alpha} \cdot\left\|u_{t}\right\| \cdot\|\psi\| .
$$


Proof. Use the boundedness of $g$, the Cauchy-Schwarz inequality, the preceding corollary, Lemma B.5, and Lemma B.6.

This corollary holds for each $\alpha \in] 0, \frac{2}{3}$ [. However we will want this contribution to be $o\left(t^{\frac{2}{3}}\right)$ so that we will need to take $\left.\alpha \in\right] 0, \frac{1}{3}[$.

B.2.3. The Airy approximation. For small $t$, the function $f_{t}^{k}$ has a simple zero near $y=1$. Thus, to approximate solutions of $t^{2} \cdot v^{\prime \prime}+f_{E_{t}}^{k} \cdot v=r$ near $y=1$ we will use solutions to Airy's differential equation $w^{\prime \prime}(x)-x \cdot w=0$ where $x=y-1$.

We first describe the link to Airy's equation. If we define $W(x):=v_{t}^{k}(x+1)$, then we have

$$
-t^{2} \cdot W^{\prime \prime}(x)+\left((k \pi)^{2}-\frac{E}{(x+1)^{2}}\right) \cdot W(x)=\tilde{r}(x) .
$$

where $\tilde{r}(x)=r(x+1)$. Let $\rho$ be the smooth function that satisfies

$$
\frac{1}{(x+1)^{2}}=1-2 x+x^{2} \cdot \rho(x) .
$$

By substituting the latter expression into 153 and by dividing by $2 E_{t}$, we find that

$$
-\frac{t^{2}}{2 E_{t}} \cdot W^{\prime \prime}+x \cdot W+\frac{1}{2} \cdot\left(\frac{(k \pi)^{2}}{E_{t}}-1\right) \cdot W=\frac{\widetilde{r}}{2 E_{t}}-\frac{x^{2}}{2} \cdot \rho \cdot W .
$$

Setting

$$
\begin{aligned}
s & =\frac{t}{\sqrt{2 E_{t}}} \\
z_{s} & =\frac{1}{2} \cdot\left(1-\frac{(k \pi)^{2}}{E_{t}}\right),
\end{aligned}
$$

we have

$$
-s^{2} \cdot W^{\prime \prime}(x)+\left(x-z_{s}\right) \cdot W(x)=R_{t}(x)
$$

where

$$
R_{t}(x)=\left(2 E_{t}\right)^{-1} \cdot \widetilde{r}(x)-2^{-1} \cdot x^{2} \cdot \rho(x) \cdot W(x) .
$$

In the next few subsections, we will analyse the solutions to 156$)$. But first, we provide an estimate of the $L^{2}\left(\left[0,3 t^{\alpha}\right]\right)$ norm of $R_{t}$.

Lemma B.10. For each $\alpha<\frac{1}{3}$, there exists $C>0$ and $t_{0}>0$ such that for each $t<t_{0}$

$$
\int_{0}^{3 t^{\alpha}}\left|R_{t}(x)\right|^{2} d x \leqslant C \cdot t^{2\left(2 \alpha+\frac{1}{3}\right)} \cdot\left\|u_{t}\right\|^{2} .
$$

Proof. We have $E \geqslant(k \pi)^{2} \geqslant 1$, and hence by Lemma B.5.

$$
\left\|\frac{r}{E}\right\|^{2} \leqslant\left\|r_{t}\right\|^{2} \leqslant C \cdot t^{2} \cdot\left\|u_{t}\right\|^{2} .
$$

Let $\psi^{*}=\psi_{t}^{*}$ be the tracking eigenvalue branch associated to $E=E_{t}$. The eigenvalue $\lambda_{t}^{*}$ corresponding to $\psi^{*}$ is in a $O(t)$ neighbourhood of $E_{t}$. Moreover, when $t$ tends to 0 , Proposition A.1 implies that $\lambda_{t}$ is at a distance of order $t^{\frac{2}{3}}$ of the rest 
of the spectrum. Using 109, (123), Lemma B.5 and a resolvent estimate, we have $\left\|v_{t}^{k}-\psi_{t}^{*}\right\|=O\left(t^{\frac{1}{3}}\right)\left\|u_{t}\right\|$ and hence

$$
\int_{0}^{3 t^{\alpha}}\left|x^{2} \cdot \rho(x) \cdot\left(v_{t}-\psi_{t}^{*}\right)(x+1)\right|^{2} d x \leqslant C \cdot t^{4 \alpha} \cdot t^{\frac{2}{3}}\left\|u_{t}\right\|^{2} .
$$

The tracking eigenfunction $\psi^{*}$ satisfies 124 with $r=0$. Hence, by Proposition B.7, if $\alpha \leqslant \widetilde{\alpha}<2 / 3$, we obtain

$$
\int_{2 t^{\widetilde{\alpha}}}^{3 t^{\alpha}}\left|x^{2} \cdot \rho(x) \cdot \psi^{*}(x+1)\right|^{2} d x \leqslant C \cdot t^{4 \alpha} \cdot \exp \left(-t^{\frac{3 \widetilde{\alpha}-2}{2}}\right)\left\|\psi^{*}\right\|^{2} .
$$

Observe that, by orthogonality, $\left\|\psi^{*}\right\| \leqslant\left\|u_{t}\right\|$; it follows

$$
\int_{0}^{3 t^{\alpha}}\left|x^{2} \cdot \rho(x) \cdot \psi^{*}(x+1)\right|^{2} d x \leqslant C^{\prime} \cdot\left(t^{4 \widetilde{\alpha}}+t^{4 \alpha} \exp \left(-t^{\frac{3 \widetilde{\alpha}-2}{2}}\right)\right) \cdot\left\|u_{t}\right\|^{2} .
$$

Since $2\left(2 \alpha+\frac{1}{3}\right)<2$ (because $\alpha<\frac{1}{3}$ ), we may thus take $\tilde{\alpha}=\frac{1}{2}$ and the biggest term is then of order $t^{2\left(2 \alpha+\frac{1}{3}\right)}$. The claim follows.

B.2.4. The inhomogeneous, semi-classical Airy equation. According to (156), an estimate of $v$ will result from estimating the solutions to

$$
-s^{2} \cdot W^{\prime \prime}+\left(x-z_{s}\right) \cdot W=R
$$

for $s^{-\frac{2}{3}} \cdot z_{s}$ in a fixed compact set.

We first construct solutions to the associated homogeneous equation

$$
-s^{2} \cdot W^{\prime \prime}+\left(x-z_{s}\right) \cdot W=0
$$

using Airy functions. In particular, it is well-known that there exists a basis $\left\{A_{+}, A_{-}\right\}$of solutions to $-A^{\prime \prime}(x)+x \cdot A(x)=0$ such that

$$
\lim _{x \rightarrow \infty} \frac{A_{ \pm}(x)}{x^{-\frac{1}{4}} \cdot \exp \left( \pm \frac{2}{3} \cdot x^{\frac{3}{2}}\right)}=1
$$

and

$$
\lim _{x \rightarrow \infty} \frac{A_{ \pm}^{\prime}(x)}{x^{\frac{1}{4}} \cdot \exp \left( \pm \frac{2}{3} \cdot x^{\frac{3}{2}}\right)}= \pm 1
$$

One checks that

$$
W_{ \pm}(x)=A_{ \pm}\left(s^{-\frac{2}{3}}\left(x-z_{s}\right)\right)
$$

defines a basis of solutions to 160 .

It follows from well-known identities that the Wronskian- $A_{+}^{\prime} A_{-}-A_{+} A_{-}^{\prime}$ - of $\left\{A_{+}, A_{-}\right\}$is 2 . Hence the Wronskian of $\left\{W_{+}, W_{-}\right\}$is $2 s^{-\frac{2}{3}}$. Therefore, by the method of variation of constants, for each $\bar{x}>0$, the function

$$
W_{\bar{x}}(x)=\frac{1}{2} \cdot s^{-\frac{4}{3}}\left(W_{+}(x) \int_{x}^{\bar{x}} R \cdot W_{-}+W_{-}(x) \int_{0}^{x} R \cdot W_{+}\right)
$$

is a solution to 159 . 
Lemma B.11. For each compact set $K \subset \mathbb{R}$, there exists $C$ such that, for each $\bar{x}>0$, if $s^{-\frac{2}{3}} \cdot z_{s} \in K$ and $x \in[0, \bar{x}]$, then

$$
\left|W_{\bar{x}}(x)\right| \leqslant C \cdot s^{-\frac{4}{3}} \cdot s^{\frac{1}{3}} \cdot\|R\|
$$

and

$$
\left|W_{\bar{x}}^{\prime}(x)\right| \leqslant C \cdot s^{-\frac{4}{3}} \cdot s^{-\frac{1}{3}} \cdot\|R\|
$$

where $\|R\|$ denotes the $L^{2}$ norm of $R$ over $[0, \bar{x}]$. Moreover, there exists $M$ so that if $x \in\left[M \cdot s^{\frac{2}{3}}, \bar{x}\right]$, then

$$
\left|W_{\bar{x}}(x)\right| \leqslant 2 \cdot s^{-\frac{4}{3}} \cdot s^{\frac{1}{3}} \cdot s^{\frac{1}{2}} \cdot\|R\| \cdot x^{-\frac{3}{4}}
$$

and

$$
\left|W_{\bar{x}}^{\prime}(x)\right| \leqslant 2 \cdot s^{-\frac{4}{3}} \cdot s^{-\frac{1}{3}} \cdot s^{\frac{1}{6}} \cdot\|R\| \cdot x^{-\frac{1}{4}} .
$$

Proof. Using the Cauchy-Schwarz inequality, we have for $x \in[0, \bar{x}]$

$$
\left|\int_{x}^{\bar{x}} R(z) \cdot W_{-}(z) d z\right| \leqslant\left(\int_{0}^{\bar{x}} R(z)^{2} d z\right)^{\frac{1}{2}} \cdot\left(\int_{x}^{\infty} W_{-}(z)^{2} d z\right)^{\frac{1}{2}}
$$

and

$$
\left|\int_{0}^{x} R(z) \cdot W_{+}(z) d z\right| \leqslant\left(\int_{0}^{\bar{x}} R(z)^{2} d z\right)^{\frac{1}{2}} \cdot\left(\int_{0}^{x} W_{+}(z)^{2} d z\right)^{\frac{1}{2}}
$$

Thus, from 164 and the triangle inequality, we have

$$
\begin{aligned}
\left|W_{\bar{x}}(x)\right| \leqslant & \frac{1}{2} s^{-\frac{4}{3}} \cdot\left(\left|W_{+}(x)\right| \cdot\|R\| \cdot\left(\int_{x}^{\infty} W_{-}^{2}\right)^{\frac{1}{2}}\right. \\
& \left.+\left|W_{-}(x)\right| \cdot\|R\| \cdot\left(\int_{0}^{x} W_{+}^{2}\right)^{\frac{1}{2}}\right)
\end{aligned}
$$

Estimate 165) then follows from Lemma B.12 below.

To prove (166) we apply a similar argument to

$$
W_{\bar{x}}^{\prime}(x)=2 \cdot s^{-\frac{4}{3}} \cdot\left(W_{+}^{\prime}(x) \int_{x}^{\bar{x}} R \cdot W_{-}+W_{-}^{\prime}(x) \int_{0}^{x} R \cdot W_{+}\right) .
$$

Define $I_{+}(x)=[0, x]$ and $I_{-}(x)=[x, \infty]$.

Lemma B.12. There exists $C$ so that if $x \geqslant 0$ and $s^{-\frac{2}{3}} \cdot z_{s} \in K$, then

$$
W_{ \pm}(x)^{2} \int_{I_{\mp}(x)} W_{\mp}(y)^{2} d y \leqslant C \cdot s^{\frac{2}{3}}
$$

and

$$
W_{ \pm}^{\prime}(x)^{2} \int_{I_{\mp}(x)} W_{\mp}(y)^{2} d y \leqslant C \cdot s^{-\frac{2}{3}} .
$$

Moreover, there exists a constant $M$ so that if $x>M \cdot s^{\frac{2}{3}}$, then

$$
W_{ \pm}(x)^{2} \int_{I_{\mp}(x)} W_{\mp}(y)^{2} d y \leqslant 4 \cdot \sqrt{2} \cdot s^{\frac{2}{3}} \cdot s \cdot x^{-\frac{3}{2}} .
$$


and

$$
W_{ \pm}^{\prime}(x)^{2} \int_{I_{\mp}(x)} W_{\mp}(y)^{2} d y \leqslant 2 \sqrt{2} \cdot s^{-\frac{2}{3}} \cdot s^{\frac{1}{3}} \cdot x^{-\frac{1}{2}} .
$$

Proof. The proof is a straightforward consequence of the continuity and known asymptotics of $A_{ \pm}$and $A_{ \pm}^{\prime}$. From 161 and integration by parts we find that

$$
\int_{I_{\mp}(u)}\left|A_{ \pm}(r)\right|^{2} d r \sim \frac{1}{2} \cdot u^{-1} \cdot \exp \left( \pm \frac{4}{3} \cdot u^{\frac{3}{2}}\right),
$$

as $u$ tends to $\infty$.

Thus there exists $u^{*}$ so that if $u \geqslant u^{*}$, then

$$
\int_{u}^{\infty} A_{-}(r)^{2} d r \leqslant u^{-1} \exp \left(-\frac{4}{3} \cdot u^{\frac{3}{2}}\right) .
$$

Therefore, for $u \geqslant u^{*}$,

$$
A_{+}(u)^{2} \int_{u}^{\infty} A_{-}(r)^{2} d r \leqslant 2 \cdot u^{-\frac{3}{2}}
$$

and, using 162,

$$
A_{+}^{\prime}(u)^{2} \int_{u}^{\infty} A_{-}(r)^{2} d r \leqslant 2 \cdot u^{-\frac{1}{2}}
$$

The expressions on the left hand sides of $(175)$ and $\sqrt{176}$ are continuous in $u$, and hence are bounded by a constant $C$ for $u \in \check{K} \cup[0, \infty[$ where $u \in \check{K} \Leftrightarrow-u \in K$.

By 163 and the change of variable $r=s^{-\frac{2}{3}} \cdot\left(y-z_{s}\right)$, we have

$$
\int_{x}^{\infty} W_{-}(y)^{2} d y=s^{\frac{2}{3}} \cdot \int_{u_{s}(x)}^{\infty} A_{-}(r)^{2} d r
$$

where $u_{s}(x)=s^{-\frac{2}{3}} \cdot\left(x-z_{s}\right)$. Since for each $x>0$ and $u_{s}(x) \geqslant-\sup K$ estimate 170 follows.

Moreover, from 175 and 176 we have

$$
W_{+}(x)^{2} \int_{u_{s}(x)}^{\infty} W_{-}(y)^{2} d y \leqslant 2 \cdot s^{\frac{2}{3}} \cdot u_{s}(x)^{-\frac{3}{2}},
$$

and

$$
W_{+}^{\prime}(x)^{2} \int_{u_{s}(x)}^{\infty} W_{-}(y)^{2} d y \leqslant 2 \cdot s^{-\frac{2}{3}} \cdot u_{s}(x)^{-\frac{1}{2}}
$$

provided $u_{s} \geqslant u^{*}$. Let $M=u^{*}+2 \sup K$. If $x>M \cdot s^{\frac{2}{3}}$, then $x-z_{s}>x / 2$ and $u_{s}(x)>u_{*}$. The desired estimates in the $+/-$ case follow. The estimates in the $-/+$ case are proved in a similar fashion.

B.2.5. The end of the proof of Lemma B.4. By 152 it suffices to estimate

$$
\int_{1}^{1+3 t^{\alpha}} g(y) \cdot v_{t}^{k}(y) \cdot(t \psi)^{\prime}(y) d y=\int_{0}^{3 t^{\alpha}} \widetilde{g}(x) \cdot W_{t}(x) \cdot(t \widetilde{\psi})^{\prime}(x) d x .
$$

where $W_{t}=v_{t}^{k}(x+1), \widetilde{g}(x)=g(x+1)$, and $\widetilde{\psi}(x)=\psi(x+1)$. By assumption, the $C^{1}$ norm of $g$ and hence $\widetilde{g}$ is uniformly bounded. 
The function $W_{t}$ satisfies the inhomogeneous equation $\sqrt{159}$ with $s=t / \sqrt{E_{t}}$, and the inhomogeneity $R_{t}$ satisfies 157). In order to estimate $W_{t}$ and hence (180), we write

$$
W_{t}=W_{p, t}+W_{h, t}
$$

where $W_{p, t}$ is taken to be the particular solution $W_{\bar{x}}$ to 159 defined by (164) where we set $\bar{x}=3 t^{\alpha}$. The function $W_{h, t}$ is then a solution to the associated homogeneous equation.

Lemma B.13. For each $\alpha \in] \frac{13}{42}, \frac{1}{3}[$ there exists $\delta>0$ such that

$$
\left|\int_{0}^{2 t^{\alpha}} \tilde{g} \cdot W_{p, t} \cdot(t \widetilde{\psi})^{\prime} d x\right| \leqslant C \cdot t^{\frac{2}{3}+\delta} \cdot\|\psi\| \cdot\|u\|
$$

for all t sufficiently small.

Proof. Integration by parts gives

$$
\int_{0}^{2 t^{\alpha}} \widetilde{g} \cdot W_{p, t} \cdot(t \widetilde{\psi})^{\prime}=\left.\widetilde{g} \cdot W_{p, t} \cdot t \widetilde{\psi}\right|_{0} ^{2 t^{\alpha}}-\int_{0}^{2 t^{\alpha}} \partial_{x}\left(\widetilde{g} \cdot W_{p, t}\right) \cdot t \widetilde{\psi} .
$$

Using Lemmas B.10 and B.11, one finds that there exists $C$ such that, for $x \in\left[0,3 t^{\alpha}\right]$

$$
\left|W_{p, t}(x)\right| \leqslant C \cdot t^{-1} \cdot t^{2 \alpha+\frac{1}{3}} \cdot\left\|u_{t}\right\|
$$

Thus, using (B.6), we conclude that the first term on the right side of (181) is $O\left(t^{2 \alpha+\frac{1}{3}}\right) \cdot\left\|u_{t}\right\| \cdot\|\psi\|$.

To bound the second term on the right side of 181 , we separately consider the integral of $\left(\partial_{x} \widetilde{g}\right) \cdot W_{p, t} \cdot t \widetilde{\psi}$ and the integral of $\widetilde{g} \cdot\left(\partial_{x} W_{p, t}\right) \cdot t \widetilde{\psi}$. For the first integral we can use the same uniform bound on $W_{p, t}$ as above to obtain a contribution that is $t^{\alpha} \cdot O\left(t^{2 \alpha+\frac{1}{3}}\right) \cdot\left\|u_{t}\right\| \cdot\|\psi\|$.

To estimate the second integral, we choose $\widetilde{\alpha}$ so that $\frac{2}{3}>\widetilde{\alpha}>\alpha$, and separately estimate the integral over $\left[0, t^{\widetilde{\alpha}}\right]$ and the integral over $\left[t^{\widetilde{\alpha}}, 3 t^{\alpha}\right]$. Observe that since $\widetilde{\alpha}<\frac{2}{3}$ then $t^{\widetilde{\alpha}}>M s^{\frac{2}{3}}$. Using Lemmas B.10 and B.11, we find $C$ so that for all sufficiently small $t$

$$
\int_{t^{\widetilde{\alpha}}}^{2 t^{\alpha}}\left|\widetilde{g} \cdot W_{p, t}^{\prime}(x)\right| d x \leqslant C \cdot t^{-\frac{3}{2}} \cdot t^{-\frac{1}{4} \cdot \widetilde{\alpha}} \cdot t^{2 \alpha+\frac{1}{3}} \cdot t^{\alpha} \cdot\left\|u_{t}\right\|,
$$

and

$$
\int_{0}^{t^{\widetilde{\alpha}}}\left|\widetilde{g} \cdot W_{p, t}^{\prime}(x)\right| d x \leqslant C \cdot t^{-\frac{5}{3}} \cdot t^{2 \alpha+\frac{1}{3}} \cdot t^{\widetilde{\alpha}} \cdot\left\|u_{t}\right\| .
$$

By combining these estimates and using (141), we find that

$$
\begin{aligned}
\int_{0}^{2 t^{\alpha}} \widetilde{g} \cdot W_{p, t} \cdot(t \cdot \widetilde{\psi})^{\prime} & \leqslant C \cdot t^{2 \alpha+\frac{1}{3}}\left\|u_{t}\right\|+C \cdot t \cdot \int_{0}^{2 t^{\alpha}}\left|\widetilde{g} \cdot W_{p, t}^{\prime}(x)\right| d x \\
& \leqslant C \cdot\left(t^{2 \alpha+\frac{1}{3}}+t^{-\frac{1}{6}+3 \alpha-\frac{1}{4} \widetilde{\alpha}}+t^{-\frac{1}{3}+2 \alpha+\widetilde{\alpha}}\right)\left\|u_{t}\right\| .
\end{aligned}
$$

The claim will follow provided we can choose $(\alpha, \widetilde{\alpha})$ so that $\alpha<\frac{1}{3}, \alpha<\widetilde{\alpha}$, and each power of $t$ appearing on the righthand side is greater than $2 / 3$. The solution set to this problem is the open triangle in $\mathbb{R}^{2}$ bounded by the lines $\alpha<1 / 3,2 \alpha+\widetilde{\alpha}=1$, and $3 \alpha-\widetilde{\alpha} / 4=5 / 6$. The two latter lines intersect for $\alpha=\frac{13}{42}$. The claim follows.

The same kind of argument allows us to estimate the norm of $W_{p, t}$ 
Lemma B.14. For all $\alpha \in] \frac{7}{33}, \frac{1}{3}[$, there exists $\delta>0$ and $C>0$ such that

$$
\left\|W_{p, t}\right\|_{\left[0,3 t^{\alpha}\right]} \leqslant C \cdot t^{\delta} \cdot\left\|u_{t}\right\| \text {. }
$$

Proof. As above we consider $\alpha<\frac{1}{3}$ and take some $\widetilde{\alpha}>\alpha$. Using Lemmas B.10 and B.11, one finds that

$$
\begin{gathered}
\left\|W_{p, t}\right\|_{\left[t^{\widetilde{\alpha}}, 3 t^{\alpha}\right]} \leqslant C \cdot t^{\frac{5 \alpha}{2}-\frac{3 \widetilde{\alpha}}{4}-\frac{1}{6}}\left\|u_{t}\right\| \\
\left\|W_{p, t}\right\|_{\left[0, t^{\widetilde{\alpha}}\right]} \leqslant C \cdot t^{2 \alpha+\frac{\widetilde{\alpha}}{2}-\frac{2}{3}}\left\|u_{t}\right\| .
\end{gathered}
$$

The claim will follow provided we can find $\alpha<\widetilde{\alpha}$ and $\alpha<\frac{1}{3}$ such that $\frac{5 \alpha}{2}-\frac{3 \widetilde{\alpha}}{4}-$ $\frac{1}{6}>0$ and $2 \alpha+\frac{\widetilde{\alpha}}{2}-\frac{2}{3}>0$. Here the solution set is a quadrilateral whose projection onto the $\alpha$-axis is the interval $] \frac{7}{33}, \frac{1}{3}[$.

Finally, we consider the integral corresponding to the homogeneous part $W_{h, t}$ of $W_{t}$ :

$$
\int_{0}^{2 t^{\alpha}} \widetilde{g}(x) \cdot W_{h, t}(x) \cdot(t \cdot \widetilde{\psi})^{\prime}(x) d x .
$$

There exist constants $a_{+}, a_{-}$, depending on $t$, such that

$$
W_{h, t}=a_{+} \cdot W_{+}+a_{-} \cdot W_{-} .
$$

where $W_{+}$and $W_{-}$are as defined in 163 with the parameter $s$ and $z_{s}$ defined in (154).

We first prove a lemma that roughly says that in the decomposition $W=W_{p, t}+$ $a_{+} W_{+}+a_{-} W_{-}$the $L^{2}$ norm is mainly supported by $a_{-} W_{-}$.

Lemma B.15. For all $\alpha \in] \frac{7}{33}, \frac{1}{3}[$, there exists $\delta>0$ such that

$$
\left\|a_{+} W_{+}\right\|_{\left[0,2 t^{\alpha}\right]}=O\left(t^{\infty}\right) \cdot\left\|u_{t}\right\|
$$

where $O\left(t^{\infty}\right)$ is a function that is of order $t^{n}$ for each $n$, and

$$
\left\|a_{-} W_{-}\right\|_{\left[0,2 t^{\alpha}\right]} \geqslant \frac{1}{2} \cdot\left(\left\|w_{t}^{k}\right\|-C \cdot t^{\delta}\left\|u_{t}\right\|\right) .
$$

Proof. Using the behavior of the norm of $A_{ \pm}$we find that

$$
\left\|a_{+} W_{+}\right\|_{\left[0,2 t^{\alpha}\right]}=O\left(t^{\infty}\right) \cdot\left\|a_{+} W_{+}\right\|_{\left[2 t^{\alpha}, 3 t^{\alpha}\right]}
$$

and

We thus have

$$
\left\|a_{-} W_{-}\right\|_{\left[2 t^{\alpha}, 3 t^{\alpha}\right]} \leqslant C \cdot\left\|a_{-} W_{-}\right\|_{\left[0,2 t^{\alpha}\right]}
$$

$$
\begin{aligned}
\left\|a_{+} W_{+}\right\|_{\left[0,2 t^{\alpha}\right]} & =O\left(t^{\infty}\right) \cdot\left\|a_{+} W_{+}\right\|_{\left[2 t^{\alpha}, 3 t^{\alpha}\right]} \\
& \leqslant O\left(t^{\infty}\right) \cdot\left(\|W\|_{\left[2 t^{\alpha}, 3 t^{\alpha}\right]}+\left\|a_{-} W_{-}\right\|_{\left[2 t^{\alpha}, 3 t^{\alpha}\right]}+\left\|W_{p, t}\right\|_{\left[2 t^{\alpha}, 3 t^{\alpha}\right]}\right) \\
& \leqslant O\left(t^{\infty}\right) \cdot\left(\left\|u_{t}\right\|+\left\|W_{-}\right\|_{\left[0,2 t^{\alpha}\right]}+t^{\delta}\left\|u_{t}\right\|\right) \\
& \leqslant O\left(t^{\infty}\right) \cdot\left(\left\|u_{t}\right\|+\|W\|_{\left[0,2 t^{\alpha}\right]}+\left\|a_{+} W_{+}\right\|_{\left[0,2 t^{\alpha}\right]}+\left\|W_{p, t}\right\|_{\left[0,2 t^{\alpha}\right]}\right) \\
& \leqslant O\left(t^{\infty}\right)\left(\left\|a_{+} W_{+}\right\|_{\left[0,2 t^{\alpha}\right]}+\left\|u_{t}\right\|\right) .
\end{aligned}
$$

Estimate (184 then follows by absorbing the norm of $a_{+} W_{+}$into the left hand side.

To prove estimate (185), we first observe that by using the triangle inequality we find that

$$
\|W\|_{\left[0,2 t^{\alpha}\right]} \leqslant\left\|W_{p, t}\right\|_{\left[0,2 t^{\alpha}\right]}+\left\|a_{-} W_{-}\right\|_{\left[0,2 t^{\alpha}\right]}+\left\|a_{+} W_{+}\right\|_{\left[0,2 t^{\alpha}\right]} .
$$


The first term on the righta hand side is $O\left(t^{\delta}\right)\left\|u_{t}\right\|$ and the last one is $O\left(t^{\infty}\right)\left\|u_{t}\right\|$ so that we obtain

$$
\left\|a_{-} W_{-}\right\|_{\left[0,2 t^{\alpha}\right]} \geqslant\left(\|W\|_{\left[0,2 t^{\alpha}\right]}-O\left(t^{\delta}\right)\left\|u_{t}\right\|\right) .
$$

The claim then follows by observing that Corollary B.8 implies that

$$
\|W\|_{\left[0,2 t^{\alpha}\right]} \geqslant \frac{1}{2}\left(\left\|w_{t}^{k}\right\|-O\left(t^{1-\alpha}\right)\left\|u_{t}\right\|\right) .
$$

Lemma B.16. We have

$$
\int_{0}^{2 t^{\alpha}} \widetilde{g} \cdot W_{-} \cdot(t \cdot \widetilde{\psi})^{\prime} d x=(\pi k) \cdot t \cdot g(1) \cdot A_{-}\left(-s^{-\frac{2}{3}} z_{s}\right)+O\left(t^{\frac{4}{3}}\right)
$$

for $t$ small.

Proof. From 145, we have

$$
\widetilde{\psi}^{\prime}(x)=-(x+1)^{-\frac{1}{2}} \cdot\left(r+\frac{1}{2 r}\right) \cdot \sin (r \cdot \ln (x+1)) .
$$

Thus, the integral we want to estimate can be written as

$$
t\left(r+\frac{1}{2 r}\right) \int_{0}^{2 t^{\alpha}} a_{0}(x) A_{-}\left(s^{-\frac{2}{3}}\left(x-z_{s}\right)\right) \sin (r \cdot \ln (x+1)) d x,
$$

where we have set $a_{0}(x):=-(x+1)^{-\frac{1}{2}} \widetilde{g}$. Denote by $I(t)$ the integral

$$
I(t)=r \int_{0}^{2 t^{\alpha}} a_{0}(x) A_{-}\left(s^{-\frac{2}{3}}\left(x-z_{s}\right)\right) \exp (i r \cdot \ln (x+1) d x .
$$

Integration by parts shows that

$$
\begin{aligned}
I(t) & =-i a_{1}(x) A_{-}\left(s^{-\frac{2}{3}}\left(x-z_{s}\right)\right) \exp \left(\left.i r \cdot \ln (x+1)\right|_{0} ^{2 t^{\alpha}}\right. \\
& -\frac{1}{i r} \int_{0}^{2 t^{\alpha}} \partial_{x}\left(a_{1}(x) A_{-}\left(s^{-\frac{2}{3}}\left(x-z_{s}\right)\right)(r \exp (i r \cdot \ln (x+1))) d x\right.
\end{aligned}
$$

where we have set $a_{1}(x)=a_{0}(x)(x+1)$.

Since $\alpha<\frac{1}{3}<\frac{2}{3}$ and $s$ is of order $t$, and since $A_{-}$is rapidly decreasing, the boundary term at $2 t^{\alpha}$ is $O\left(t^{\infty}\right)$. Observe that we have a global $\frac{1}{r}$ prefactor in front of the integral term. Thus, when the $\partial_{x}$ is applied to $a_{1}$, we gain $1 / r$, that is, something of order $t$. When $\partial_{x}$ hits the Airy function, we lose a $s^{-\frac{2}{3}}$ so that the global prefactor is of order $\frac{s^{-\frac{2}{3}}}{r}$ which is $O\left(t^{\frac{1}{3}}\right)$. Summarizing, integrating by parts gains at least a prefactor $t^{\frac{1}{3}}$.

By repeated integration by parts we thus observe that we can write, for each $N$

$$
I(t)=\sum_{n=0}^{N-1} \sum_{k+\ell=n} r^{-k}\left(\frac{s^{-\frac{2}{3}}}{r}\right)^{\ell} a_{k, \ell} A^{(\ell)}\left(-s^{-\frac{2}{3}} z_{s}\right)+R_{N}+O\left(t^{\infty}\right),
$$

where the $a_{k, \ell}$ are some constants and the remainder term $R_{N}$ can be written

$$
R_{N}(t):=\sum_{k+\ell=N} r^{-k}\left(\frac{s^{-\frac{2}{3}}}{r}\right)^{\ell} \int_{0}^{2 t^{\alpha}} a_{k, \ell}(x) A_{-}^{\ell}\left(s^{-\frac{2}{3}}\left(x-z_{s}\right)\right)(r \exp (i r \cdot \ln (x+1))) d x
$$


for some smooth functions $a_{k, \ell}$. If we fix some order $t^{M}$ then, using that $A_{-}$and all its derivatives are rapidly decreasing, we can find $N$ such that the remainder $R_{N}$ is $O\left(t^{N}\right)$. This tells us that $I(t)$ admits a complete asymptotic expansion of the form

$$
I(t) \sim a_{00} r^{-1} A\left(-s^{-\frac{2}{3}} z_{s}\right)+\sum_{k, l \geqslant 1} a_{k, l} r^{-k}\left(\frac{s^{-\frac{2}{3}}}{r}\right)^{\ell} a_{k \ell} A^{(\ell)}\left(-s^{-\frac{2}{3}} z_{s}\right) .
$$

From the first integration by parts we see that

$$
a_{00}=i \widetilde{g}(0)
$$

and the second term is then of order $t^{\frac{1}{3}}$. The claim follows by taking the imaginary part.

We will use the following to verify that the leading order term does not vanish.

Lemma B.17. We have

$$
\lim _{s \rightarrow 0} s^{-\frac{2}{3}} \cdot z_{s}=-\zeta
$$

where $-\zeta$ is a zero of the derivative of $A_{-}$.

Proof. From 154 we have

$$
s^{-\frac{2}{3}} \cdot z_{s}=\frac{E_{t}-(\pi k)^{2}}{2^{\frac{2}{3}} \cdot t^{\frac{2}{3}} \cdot E_{t}^{\frac{2}{3}}} .
$$

By combining Lemma 8.3 and Lemma A.1 we have

$$
E_{t}-(\pi k)^{2}=2^{\frac{2}{3}} \cdot(\pi k)^{\frac{4}{3}} \cdot(-\zeta) \cdot t^{\frac{2}{3}}+O(t) .
$$

where $\zeta$ is a zero of $A_{-}^{\prime}$. Since $\lim _{t \rightarrow 0} E_{t}=(\pi k)^{2}$, the claim follows.

Corollary B.18. There exists $\kappa^{\prime}>0$ and $t_{0}>0$ so that if $t<t_{0}$, then

$$
\left|\int_{0}^{3 t^{\alpha}} \widetilde{g}(x) \cdot W_{-}(x) \cdot(t \cdot \widetilde{\psi})^{\prime}(x) d x\right| \geqslant \kappa^{\prime} \cdot t^{\frac{2}{3}} \cdot\left\|W_{-}\right\|
$$

for $t$ sufficiently small.

Proof. Let $\zeta$ be the zero of $A_{-}$that comes from Lemma B.17 Since $A_{-}$is a nontrivial solution to a second order differential equation, $A_{-}$can not vanish at a zero of the derivative $A_{-}^{\prime}$. Hence, for sufficiently for small $t$, we have $\left|A_{-}\left(-s^{\frac{2}{3}} \cdot z_{s}\right)\right|>$ $\frac{1}{2}\left|A_{-}(\zeta)\right|>0$.

By arguing as in the proof of Lemmas B.17 and B.12 and using $s \sim t$, we find $c_{1}>0$ so that

$$
\int_{0}^{\beta-1} W_{-}(x)^{2} d x \geqslant \frac{1}{4} \cdot c_{1} \cdot t^{\frac{2}{3}}
$$

where $k_{1}=\int_{-\sup (K)}^{\infty}\left|A_{-}(u)\right|^{2}$ and $t$ is sufficiently small. In particular,

$$
1 \leqslant \frac{2}{\sqrt{c_{1}}} \cdot t^{-\frac{1}{3}} \cdot\left\|W_{-}\right\| .
$$

Hence the claim follows from Lemma B.16. 
The estimate in the latter corollary is homogeneous so that we can multiply $W_{-}$ by $a_{-}$.

Using Lemma B.15 we then have

$\left|\int_{0}^{2 t^{\alpha}} \widetilde{g}(x) \cdot a_{-} W_{-}(x) \cdot(t \cdot \widetilde{\psi})^{\prime}(x) d x\right| \geqslant \kappa^{\prime} \cdot t^{\frac{2}{3}} \cdot\left\|a_{-} W_{-}\right\| \geqslant \frac{\kappa^{\prime}}{2} \cdot t^{\frac{2}{3}}\left(\left\|w_{t}^{k}\right\|-t^{\delta}\left\|u_{t}\right\|\right)$

and

$$
\left|\int_{0}^{2 t^{\alpha}} \widetilde{g}(x) \cdot a_{+} W_{+}(x) \cdot(t \cdot \widetilde{\psi})^{\prime}(x) d x\right| \leqslant O\left(t^{\infty}\right) \cdot\left\|u_{t}\right\|
$$

Putting all the different pieces together yields the estimate.

\section{REFERENCES}

[Bergeron11] N. Bergeron, The spectrum of hyperbolic surfaces. Universitext. Springer, 2016.

[BorFre10] D. Borisov and P. Freitas, Asymptotics of Dirichlet eigenvalues and eigenfunctions of the Laplacian on thin domains in $\mathbf{R}^{d}$. J. Funct. Anal. 258 (2010), no. 3, 893-912.

[ColinDeVerdière82] Y. Colin de Verdière, Pseudo-laplaciens. I. Ann. Inst. Fourier 32 (1982), no. 3 , xiii, 275-286.

[ColinDeVerdière83] Y. Colin de Verdiere, Pseudo-laplaciens. II. Ann. Inst. Fourier 33 (1983), no. $2,87-113$.

[Dieudonné] J. Dieudonné, Foundations of modern analysis. Pure and Applied Mathematics, Vol. X Academic Press, New York-London 1960.

[DIPS85] J. Deshouillers, H. Iwaniec, R.S, Phillips,and P. Sarnak, Maass cusp forms. Proc. Nat. Acad. Sci. U.S.A. 82 (1985), no. 11, 3533-3534.

[FriSol09] L. Friedlander and M. Solomyak, On the spectrum of the Dirichlet Laplacian in a narrow strip. Israel J. Math. 170 (2009), 337-354.

[GriJer96] D. Grieser and D. Jerison, Asymptotics of the first nodal line of a convex domain. Invent. Math. 125 (1996), no. 2, 197-219.

[Hejhal92] D. A. Hejhal, Eigenvalues of the Laplacian for Hecke triangle groups. Mem. Amer. Math. Soc. 97 (1992), no. 469, vi+165 pp.

[HlrJdg09] L. Hillairet and C. Judge, Generic spectral simplicity of polygons. Proc. Amer. Math. Soc. 137 (2009), no. 6, 2139-2145.

[HlrJdg11] L. Hillairet and C. Judge, Spectral simplicity and asymptotic separation of variables. Comm. Math. Phys. 302 (2011), no. 2, 291-344.

[HlrJdg12] L. Hillairet and C. Judge, Erratum to: Spectral simplicity and asymptotic separation of variable. Comm. Math. Phys. 311 (2012), no. 3, 839-842.

[Hörm] L. Hörmander, An introduction to complex analysis in several variables. Third edition. North-Holland Mathematical Library, 7. North-Holland Publishing Co., Amsterdam, 1990.

[Jdg95] C. M. Judge. On the existence of Maass cusp forms on hyperbolic surfaces with cone points. J. Amer. Math. Soc. 8 (1995), no. 3, 715-759

[Jdg07] C. M. Judge. Small eigenvalues and maximal laminations on complete surfaces of negative curvature. In the tradition of Ahlfors-Bers. IV, 93-105, Contemp. Math., 432, Amer. Math. Soc., Providence, RI, 2007.

[Kato] T. Kato, Perturbation Theory for Linear Operators, Springer-Verlag Classics in Mathematics (1995).

[LaxPhl] P. Lax and R. Phillips, Scattering theory for automorphic forms, Princeton U. Press, 1976.

[LaxPhl80] P. Lax and R. Phillips, Scattering theory for automorphic functions. Bull. Amer. Math. Soc. (N.S.) 2 (1980), no. 2, 261-295.

[Olver] F. W. J. Olver, Asymptotics and Special Functions, AKP Classics, New York, Academic Press, (1974).

[PhlSrn85] R. S. Phillips and P. Sarnak, On cusp forms for co-finite subgroups of PSL $(2, R)$. Invent. Math. 80 (1985), no. 2, 339-364.

[PhlSrn92a] R. S. Phillips and P. Sarnak, Perturbation theory for the Laplacian on automorphic functions. J. Amer. Math. Soc. 5 (1992), no. 1, 132. 
[PhlSrn92b] R. Phillips and P. Sarnak, Automorphic spectrum and Fermi's golden rule. J. Anal. Math. 59 (1992), 179-187.

[PhlSrn94] R. Phillips and P. Sarnak, Cusp forms for character varieties. Geom. Funct. Anal. 4 (1994), no. 1, 93-118.

[Sarnak03] P. Sarnak, Spectra of hyperbolic surfaces. Bull. Amer. Math. Soc. (N.S.) 40 (2003), no. $4,441-478$

[Sarnak] P. Sarnak, Prime Geodesic Theorems. Thesis. Stanford University, 1980.

[Selberg] A. Selberg, Harmonic Analysis in Collected papers. Vol. I. Springer-Verlag, Berlin, 1989.

[Wolpert92] Scott Wolpert. Spectral limits for hyperbolic surfaces. I, II. Invent. Math. 108 (1992), no. 1, 67-89, 91-129.

[Wolpert94] S. Wolpert, Disappearance of cusp forms in special families. Ann. of Math. (2) 139 (1994), no. 2, 239-291. 